\title{
HOMOGENEOUS RANDOM MEASURES AND A WEAK ORDER FOR THE EXCESSIVE MEASURES OF A MARKOV PROCESS
}

\section{P. J. FITZSIMMONS}

\begin{abstract}
Let $X=\left(X_{t}, P^{x}\right)$ be a right Markov process and let $m$ be an excessive measure for $X$. Associated with the pair $(X, m)$ is a stationary strong Markov process $\left(Y_{t}, Q_{m}\right)$ with random times of birth and death, with the same transition function as $X$, and with $m$ as one dimensional distribution. We use $\left(Y_{t}, Q_{m}\right)$ to study the cone of excessive measures for $X$. A "weak order" is defined on this cone: an excessive measure $\xi$ is weakly dominated by $m$ if and only if there is a suitable homogeneous random measure $\kappa$ such that $\left(Y_{t}, Q_{\xi}\right)$ is obtained by "birthing" $\left(Y_{t}, Q_{m}\right)$, birth in $[t, t+d t]$ occurring at rate $\kappa(d t)$. Random measures such as $\kappa$ are studied through the use of Palm measures. We also develop aspects of the "general theory of processes" over $\left(Y_{t}, Q_{m}\right)$, including the moderate Markov property of $\left(Y_{t}, Q_{m}\right)$ when the arrow of time is reversed. Applications to balayage and capacity are suggested.
\end{abstract}

1. Introduction. Let $\left(P_{s}: s \geq 0\right)$ be a Borel right semigroup on a Lusin state space $(E, \mathcal{E})$ and let $\left(X_{t}, P^{x}\right)$ denote the associated right continuous strong Markov process. Recall that a measure $m$ on $(E, \mathcal{E})$ is excessive for $\left(P_{s}\right)$ if $m$ is $\sigma$-finite and if $m P_{s} \leq m$ for all $s>0$. Since $\left(P_{s}\right)$ is a right semigroup, one then has $m P_{s} \uparrow m$ as $s \downarrow 0$, as is well known. Let Exc denote the convex cone of excessive measures for $\left(P_{s}\right)$. Given $m \in$ Exc, according to a theorem of Kuznetsov [31], one can construct a stationary process $\left(Y_{t}: t \in \mathbf{R}\right)$ having random birth and death times and a $\sigma$-finite governing measure $Q_{m}$, such that for $t_{1}<t_{2}<\cdots<t_{n}\left(t_{i} \in \mathbf{R}\right)$

$$
\begin{aligned}
& Q_{m}\left(Y_{t_{1}} \in d x_{1}, Y_{t_{2}} \in d x_{2}, \ldots, Y_{t_{n}} \in d x_{n}\right) \\
& \quad=m\left(d x_{1}\right) P_{t_{2}-t_{1}}\left(x_{1}, d x_{2}\right) \cdots P_{t_{n}-t_{n-1}}\left(x_{n-1}, d x_{n}\right) .
\end{aligned}
$$

The process occupies a cemetery state $\Delta$ prior to the birth time $\alpha$, and subsequent to the death time $\beta$. The process $\left(Y_{t}, Q_{m}\right)$ has proved to be quite useful in studying certain aspects of the process $\left(X_{t}, P^{x}\right)$. See, for example, $[\mathbf{1}, \mathbf{1 4}, \mathbf{1 5}, \mathbf{1 6}, \mathbf{2 9}, \mathbf{3 0}$, 36, 38, 39]. In particular, $\left(Y_{t}, Q_{m}\right)$ was used to study the convex cone Exc in $[\mathbf{1 6}$, $19,30]$.

In [19] certain partial orders on Exc were studied and our purpose in this paper is to study these matters further. There are two partial orders on Exc of interest. Recall that $\xi \in$ Exc is strongly (or specifically) dominated by $m \in$ Exc provided there exists $\gamma \in$ Exc such that $\xi+\gamma=m$. In this case we write $\xi \prec m$. Since $Q_{\xi+\gamma}=Q_{\xi}+Q_{\gamma}$, it is quite easy to see that

$$
\xi \prec m \quad \text { iff } \quad Q_{\xi} \leq Q_{m} .
$$

The simple order on Exc is defined as follows: we say that $\xi$ is simply dominated by $m$, and write $\xi \leq m$, provided $\xi(A) \leq m(A)$ for all $A \in \mathcal{E}$. The proper analogue

Received by the editors October 8, 1986.

1980 Mathematics Subject Classification (1985 Revision). Primary 60J45; Secondary 60J25, 60J55. 
of (1.2) for the simple order on Exc was the point of departure for the present study. Note that if $\xi$ and $m$ are potentials (that is, if $\xi=\mu U$ and $m=\nu U$ where $\mu$ and $\nu$ are measures on $(E, \mathcal{E})$ and $U=\int_{0}^{\infty} P_{s} d s$ is the potential kernel of $X$ ) then $\mu U \leq \nu U$ iff $\mu \dashv \nu$, where $\dashv$ is the "balayage" order relative to $X$. The work of Rost [43] on balayage and Skorohod stopping suggests that for general $\xi$ and $m$ in Exc one has

$$
\xi \leq m \quad \text { iff } \quad Q_{\xi}(F)=Q_{m}\left(\int_{\mathbf{R}} F \circ b_{s} \kappa(d s)\right)
$$

for all measurable functions $F \geq 0$ on path space, where $\kappa$ is a suitable homogeneous random measure and $b_{s}$ is the "birthing" operator which deletes the portion of $\left(Y_{t}\right)$ prior to time $s$. More intuitively, the stationary process $\left(Y_{t}, Q_{\xi}\right)$ is obtained by randomly birthing the process $\left(Y_{t}, Q_{m}\right)$, the "birth intensity" at time $s$ being $\kappa(d s)$.

Of course, implicit in (1.3) is a rather strong integrability condition on the random measure $\kappa$. Indeed, since $m$ is $\sigma$-finite we may choose $f$ on $E$, measurable and strictly positive, such that $m(f)<\infty$. By $(1.1), \xi(f)=Q_{\xi}\left(f\left(Y_{t}\right)\right)$, $m(f)=Q_{m}\left(f\left(Y_{t}\right)\right)$ for any $t \in \mathbf{R}$, so that, using (1.3),

$$
\xi(f)=Q_{m}\left(f\left(Y_{t}\right) \kappa(]-\infty, t[)\right) \leq m(f)<\infty .
$$

In lifting the restrictive inequality in (1.4) we are led to define the weak order on Exc: we say that $\xi \in$ Exc is weakly dominated by $m \in$ Exc provided there is a homogeneous random measure $\kappa$, "optional" in a suitable sense, such that

$$
Q_{\xi}(F)=Q_{m}\left(\int_{\mathbf{R}} F \circ b_{s} \kappa(d s)\right) ;
$$

we write $\xi \leftarrow m$ in this case. Our main results provide necessary and sufficient conditions for a representation of the form (1.5). These results can be found in $\S 6$, as can a precise definition of $\leftarrow$. For example, we show that $\xi \leftarrow m$ iff $\xi=\sum_{n=1}^{\infty} \xi_{n}$ where $\xi_{n} \leq m, \xi_{n} \in$ Exc for each $n$. This "quasi-boundedness" condition is an analogue of the well-known result that a positive supermartingale is of the class $D$ iff it can be decomposed as a sum of positive supermartingales, each bounded by 1. Indeed, another of our necessary and sufficient conditions for (1.5) amounts to the statement that $\xi<<m$ and that $\left((d \xi / d m)\left(Y_{-t}\right): t \in \mathbf{R}\right)$ under $Q_{m}$ is a "class $D$ supermartingale with random birth and death". The reader familiar with the work of Azema [2] will not be surprised by this last assertion, since (1.5) is a time reversed version of his famous killing formula for $h$-transforms.

The machinery needed to prove the results of $\S 6$ is assembled in $\S \S 2$ through 5 . Actually, $\S 2$ serves to set our notation and to recall several results from [19]. In $\S 3$ some of the "general theory of processes" for $\left(Y_{t}, Q_{m}\right)$ is established. Most of this material consists of refinements of results to be found in [14 and 38]. In $\S 4$ a dual process $\left(\hat{X}, \hat{P}^{x}\right)$, relative to a given $m \in$ Exc, is introduced. The semigroup of $\hat{X}$ provides the transition function for $\left(Y_{t}, Q_{m}\right)$ when the arrow of time is reversed. The actual construction of $\left(\hat{X}, \hat{P}^{x}\right)$, which is adapted from Azema [2], can be found in an appendix to the paper. As one of several applications of $\left(\hat{X}, \hat{P}^{x}\right)$, we obtain the "regularity" of suitable versions of the density $d \xi / d m$ mentioned earlier. In $\S 5$ we develop some of the theory of homogeneous random measures over $\left(Y_{t}, Q_{m}\right)$. The novelty here is that to obtain representations such as (1.5) one needs to allow random measures that charge the birth time $\alpha$. Of particular importance in $\S 5$ 
is the notion of a Palm measure associated with a given homogeneous random measure. In the context of the stationary process $\left(Y_{t}, Q_{m}\right)$, similar objects have been considered in [1]; our result characterizing the class of Palm measures is the analogue of a result of Geman and Horowitz [22] in a somewhat different context.

Finally, in $\S 7$ we apply the weak order in developing a new maximum principle for excessive measures. This result is an analogue of the classical statement that if the (bounded) Green's potential $U \mu$ of a measure $\mu$ is dominated by 1 on its (compact) support, then it is dominated by 1 everywhere. Unlike the classical bounded maximum principle (which is equivalent to Hunt's hypothesis $(\mathrm{H})$, at least when all excessive functions are lower semicontinuous) our maximum principle is quite general.

As a rule we use standard notation (for which consult $[\mathbf{6}, \mathbf{1 2}, \mathbf{2 4}]$ ). The special notation for $\left(Y_{t}, Q_{m}\right)$ is recorded in the next section. In particular, if $(F, \mathcal{F})$ is a measurable space, then $b^{\mathcal{F}}$ (resp. $\mathcal{F}^{+}$) denotes the class of bounded (resp. nonnegative) real-valued $\mathcal{F}$-measurable functions on $F$. If $A \subset F$ then $\mathcal{F} \cap A$ denotes the trace of $\mathcal{F}$ on $A$; that is $\mathcal{F} \cap A=\{B \cap A: B \in \mathcal{F}\}$. If $(E, \mathcal{E})$ and $(F, \mathcal{F})$ are measurable spaces, then a kernel from $(E, \mathcal{E})$ to $(F, \mathcal{F})$ is a function $N=N(x, A)$ $(x \in E, A \in \mathcal{F})$ such that $x \rightarrow N(x, A)$ is $\mathcal{E}$-measurable for each $A \in \mathcal{F}$, and such that $A \rightarrow N(x, A)$ is a (positive) measure on $(F, \mathcal{F})$ for each $x \in E$. We use the abbreviations rc and lc for right continuous and left continuous. We use the standard notation for stochastic intervals: if $S$ and $T$ are maps from $W$ (path space) to $\mathbf{R} \cup\{-\infty,+\infty\}$, then, for example, $\rrbracket S, T \rrbracket=\{(t, w) \in \mathbf{R} \times W: S(w)<t \leq T(w)\}$, and $\llbracket S \rrbracket=\llbracket S, S \rrbracket$. Note that we always have $\rrbracket S, T \rrbracket \subset \mathbf{R} \times W$. The Borel subsets of $\mathbf{R}$ are denoted $B$.

2. Preliminaries. In this section we set the basic notation and recall several results from [19]. Let $E$ be a Borel subset of a compact metric space and let $\mathcal{E}$ denote the Borel subsets of $E$. Let $\Delta \notin E$ be the customary cemetery point and set $E_{\Delta}=E \cup\{\Delta\}, \mathcal{E}_{\Delta}=\mathcal{E} \vee\{\Delta\}$. As usual a function $f \in \mathcal{E}$ is extended to $E_{\Delta}$ by setting $f(\Delta)=0$. Let $\left(P_{s}: s \geq 0\right)$ be a Borel right semigroup on $(E, \mathcal{E})$. That is, each $P_{s}$ is a sub-Markov kernel on $(E, \mathcal{E})$ and $\left(P_{s}\right)$ satisfies the "hypotheses droites" (HD1) and (HD2) of [24].

For our purposes the strong Markov process $X$ associated with $\left(P_{s}\right)$, and the stationary process $\left(Y, Q_{m}\right)$, are conveniently realized on path spaces defined as follows. Let $W$ denote the space of paths $w: \mathbf{R} \rightarrow E_{\Delta}$ which are $E$-valued and rc on an open interval $] \alpha(w), \beta(w)[\subset \mathbf{R}$ and which take the value $\Delta$ elsewhere. The "birth" and "death" times $\alpha$ and $\beta$ are defined by

$$
\alpha(w)=\inf \{t: w(t) \in E\}, \quad \beta(w)=\sup \{t: w(t) \in E\},
$$

where inf $\phi=+\infty, \sup \phi=-\infty$. Accordingly, the constant path $[\Delta]: t \rightarrow \Delta$ satisfies $\alpha([\Delta])=+\infty, \beta([\Delta])=-\infty$. The coordinate mappings on $W$ are denoted by $Y_{t}, t \in \mathbf{R}$, and the associated $\sigma$-fields are

$$
\mathcal{G}^{0}=\sigma\left\{Y_{s}: s \in \mathbf{R}\right\}, \quad \mathcal{G}_{t}^{0}=\sigma\left\{Y_{s}: s \leq t\right\} .
$$

Killing and birthing operators are defined on $W$ by

$$
\begin{aligned}
\left(k_{t} w\right)(s) & =w(s), & & s<t, \\
& =\Delta, & & s \geq t ; \\
\left(b_{t} w\right)(s) & =w(s), & & s>t, \\
& =\Delta, & & s \leq t .
\end{aligned}
$$


We also define two families $\left(\sigma_{t}\right)$ and $\left(\tau_{t}\right)$ of shift operators:

$$
\begin{aligned}
& \left(\sigma_{t} w\right)(s)=w(t+s), \quad s, t \in \mathbf{R} ; \\
& \tau_{t} w=\sigma_{t} b_{t} w=b_{0} \sigma_{t} w, \quad t \in \mathbf{R} .
\end{aligned}
$$

Of the various identities satisfied by these operators we note the following:

$$
k_{t} k_{s}=k_{t \wedge s}, \quad b_{t} b_{s}=b_{t \vee s}, \quad \sigma_{t} \sigma_{s}=\sigma_{t+s}, \quad \tau_{t} \sigma_{s}=\tau_{t+s} .
$$

Put $\Omega=\left\{w \in W: \alpha(w)=0, Y_{0+}(w)\right.$ exists in $\left.E\right\} \cup\{[\Delta]\}$ and for $s \geq 0$ let $X_{s}, \theta_{s}, \zeta$ denote the restrictions of $Y_{s+}, \tau_{s}, \beta \vee 0$ to $\Omega$. Set $\mathcal{F}^{0}=\mathcal{G}^{0} \cap \Omega, \mathcal{F}_{t}^{0}=\mathcal{G}_{t}^{0} \cap \Omega$. Our hypotheses on the semigroup $\left(P_{s}\right)$ amount to the existence of a Borel measurable family $\left\{P^{x}: x \in E_{\Delta}\right\}$ of probability measures on $\left(\Omega, \mathcal{F}^{0}\right)$ such that the collection $X=\left(\Omega, \mathcal{F}^{0}, \mathcal{F}_{t+}^{0}, \theta_{t}, X_{t}, P^{x}\right)$ is a strong Markov realization of $\left(P_{s}\right)$. Clearly $P^{\Delta}=$ $\varepsilon_{[\Delta]}$, since $\Delta$ is a trap for $X$.

Following Dynkin we say that a family $\nu=\left(\nu_{t}: t \in \mathbf{R}\right)$ of $\sigma$-finite measures on $\mathcal{E}$ is an entrance rule provided $\nu_{t} P_{s} \leq \nu_{t+s}$ for $t \in \mathbf{R}, s>0$, and $\nu_{t} P_{s} \uparrow \nu_{t}$ as $s \downarrow 0$. An entrance law at $r \in \mathbf{R}$ is an entrance rule satisfying the additional conditions $\nu_{t}=0, t \leq r$, and $\nu_{t} P_{s}=\nu_{t+s}$ for $t>r, s>0$. An entrance law at 0 is simply an entrance law. Recall that an excessive measure is a $\sigma$-finite measure $m$ on $\mathcal{E}$ satisfying $m P_{s} \leq m$ for all $s \geq 0$. One then has $m P_{s} \uparrow m$ as $s \downarrow 0$ (see e.g., [27]); consequently $\nu_{t}=m, t \in \mathbf{R}$ defines an entrance rule. Let Exc denote the convex cone of excessive measures. The basic existence result that follows is an easy consequence of a theorem of Kuznetsov [31]. See also Getoor and Glover [28] for a nice discussion of this result.

(2.1) THEOREM. Let $\nu=\left(\nu_{t}\right)$ be an entrance rule. Then there exists a unique measure $Q_{\nu}$ on $\left(W, g^{0}\right)$ which is carried by $W \backslash\{[\Delta]\}$ and under which $\left(Y_{t}: t \in \mathbf{R}\right)$ is Markovian with transition functions $\left(P_{s}\right)$ and one-dimensional distributions $\left(\nu_{t}\right)$. More precisely, for $t \in \mathbf{R}, f \in \mathcal{E}^{+}, F \in\left(\mathcal{F}^{0}\right)^{+}$, we have

$$
\begin{gathered}
Q_{\nu}\left(f \circ Y_{t}\right)=\nu_{t}(f), \\
Q_{\nu}\left(F \circ \tau_{t} \mid \mathcal{G}_{t+}^{0}\right)=P^{Y_{t}}(F) \text { a.s. } Q_{\nu} \quad \text { on }\{\alpha<t\} .
\end{gathered}
$$

The measure $Q_{\nu}$ is necessarily $\sigma$-finite.

If $\nu_{t}=m, \forall t \in \mathbf{R}$, where $m \in$ Exc, then $Q_{\nu}$ is denoted $Q_{m}$. In this case $Q_{m}$ is stationary: $\sigma_{s}\left(Q_{m}\right)=Q_{m}$ for all $s \in \mathbf{R}$.

(2.2) REMARKS. (a) In the sequel we refer to $Q_{\nu}$ as the Kuznetsov measure associated with the entrance rule $\nu$.

(b) It is shown in [30] that if $\nu$ is an entrance law at $r$, then $Q_{\nu}(\alpha \neq r)=0$, whence the nomenclature.

(c) Because of the uniqueness of $Q_{\nu}$, if $\mu$ and $\nu$ are entrance rules then $Q_{\mu+\nu}=$ $Q_{\mu}+Q_{\nu}$. In particular, $Q_{m_{1}+m_{2}}=Q_{m_{1}}+Q_{m_{2}}$ if $m_{1}, m_{2} \in$ Exc.

Let $\nu$ be an entrance rule and let $\mathcal{N}^{\nu}$ denote the class of $Q_{\nu}$ null sets in the $Q_{\nu}$ completion of $\mathcal{G}^{0}$. Set $\mathcal{G}^{\nu}=\mathcal{G}^{0} \vee \mathcal{N}^{\nu}$ and $\mathcal{G}_{t}^{\nu}=\mathcal{G}_{t}^{0} \vee \mathcal{N}^{\nu}$. Then $\left(\mathcal{G}_{t}^{\nu}: t \in \mathbf{R}\right)$ is a right continuous filtration and $\left(Y_{t}, \mathcal{G}_{t}^{\nu}, Q_{\nu}\right)$ is a strong Markov process. That is, if $T: W \rightarrow \mathbf{R} \cup\{-\infty,+\infty\}$ satisfies $\{T \leq t\} \in \mathcal{G}_{t}^{\nu}$ for all $t \in \mathbf{R}$, then

$$
Q_{\nu}\left(F \circ \tau_{T} \mid \mathcal{G}_{T}^{\nu}\right)=P^{Y_{T}}(F) \text { a.s. } Q_{\nu} \text { on }\{\alpha<T<\beta\},
$$

for any $F \in\left(\mathcal{F}^{0}\right)^{+}$. See Mitro [36] for a proof that adapts to the present situation. It is implicit in (2.3) that $Q_{\nu}$ is $\sigma$-finite on $\mathcal{G}_{T}^{\nu} \cap\{\alpha<T<\beta\}$. 
We now recall two Riesz-type decompositions of $m \in$ Exc from [19]. Firstly, each $m \in$ Exc has a unique decomposition $m=m_{i}+m_{p}$ where $m_{i}$ is invariant (i.e., $m P_{s}=m, \forall s>0$ ) and $m_{p}$ is purely excessive (i.e., $m(f)<\infty$ implies $m P_{s}(f) \downarrow 0$ as $s \uparrow+\infty)$. The Kuznetsov measure $Q_{m}$ can be decomposed accordingly: $Q_{m}=$ $Q_{m_{i}}+Q_{m_{p}}$, where $Q_{m_{i}}=Q_{m}(\cdot ; \alpha=-\infty), Q_{m_{p}}=Q_{m}(\cdot ; \alpha>-\infty)$. Moreover $m_{p}$ admits an integral representation

$$
m_{p}=\int_{0}^{\infty} \nu_{t} d t
$$

where $\nu=\left(\nu_{t}\right)$ is a uniquely determined entrance law (at 0). By $[\mathbf{1 9},(2.4)]$ we have

$$
Q_{m}\left(\phi\left(\alpha, \tau_{\alpha}\right) ; \alpha \in \mathbf{R}\right)=\iint_{\mathbf{R} \times W} \phi(t, w) d t Q_{\nu}(d w)
$$

if $\phi \in\left(B \times \mathcal{G}^{0}\right)^{+}$. Given $F \in\left(\mathcal{G}^{0}\right)^{+}$, if we set $\phi(t, w)=F\left(\sigma_{-t} w\right)$ then $F(w)=$ $\phi\left(\alpha(w), \tau_{\alpha} w\right)$ if $\alpha(w) \in \mathbf{R}$. Applying (2.5) we obtain

$$
Q_{m_{p}}(F)=Q_{m}(F ; \alpha \in \mathbf{R})=\int_{\mathbf{R}} Q_{\nu}\left(F \circ \sigma_{-t}\right) d t .
$$

Also, taking $\phi(t, w)=1_{] 0,1]}(t) f\left(X_{s}(w)\right)$ in (2.5), we obtain

$$
\nu_{s}(f)=Q_{m}\left(f \circ Y_{\alpha+s} ; 0<\alpha \leq 1\right), \quad s>0 .
$$

In the sequel we let Inv and Pur denote the classes of invariant and purely excessive measures respectively.

A second Riesz decomposition considered in [19] concerns measure potentials. Recall that $m \in$ Exc is a potential ( $m \in$ Pot) provided $m=\mu U$ for some (necessarily $\sigma$-finite) measure $\mu$ on $\mathcal{E}$. Here $U$ denotes the potential kernel: $U=\int_{0}^{\infty} P_{t} d t$. The class Pot of potentials is characterized in [19] as follows. Let $d$ denote a metric on $E$ compatible with its topology and let $C_{u}(E)$ denote the class of bounded $d$-uniformly continuous functions from $E$ to $\mathbf{R}$. Then $C_{u}(E)$ is separable in the uniform norm. Let $D$ denote a countable dense subset of $C_{u}(E)^{+}$. Let $U^{r}=\int_{0}^{\infty} e^{-r t} P_{t} d t$. Given $q \in \mathcal{E}^{+}$with $q>0$, let $h=U q$ and define $\Omega_{q} \in \mathcal{F}^{0}$ by

$$
\begin{aligned}
\Omega_{q}=\{\alpha= & 0, Y_{0+} \text { exists in } E, \phi\left(Y_{1 / n}\right) \rightarrow \phi\left(Y_{0+}\right) \\
& \text { as } \left.n \rightarrow \infty, \text { for } \phi=h \text { and } \phi=U^{r} g, \forall g \in D, \forall r>0 \text { and rational }\right\} .
\end{aligned}
$$

The following result is (3.3) of [19]. Note that Pot $\subset$ Pur, for if $m=\mu U$ and $m(f)<\infty$ where $f \in \mathcal{E}^{+}$, then $m P_{s} f=\int_{s}^{\infty} \mu P_{t} f d t \downarrow 0$ as $s \uparrow+\infty$.

(2.8) ThEOREM. Let $m \in$ Pur, so that $m=\int_{0}^{\infty} \nu_{t} d t$ where $\nu=\left(\nu_{t}\right)$ is an entrance law. Let $Q_{\nu}$ denote the associated Kuznetsov measure. Then $m \in$ Pot if and only if $Q_{\nu}$ is carried by $\Omega_{q}$, where $q \in \mathcal{E}^{+}, q>0$, and $m(q)<\infty$. In this case $Q_{\nu}=P^{\mu}$ where $\mu=Y_{0+}\left(Q_{\nu}\right)$. In particular $m=\mu U, \nu_{t}=\mu P_{t}$, and $\left(Y_{t+}\right)_{t \geq 0}$ under $Q_{\nu}$ is a strong Markov process.

Given $m \in$ Exc we may apply (2.8) to $m_{p}$ : making use of (2.5) we see that $m_{p} \in$ Pot if and only if $Q_{m_{p}}$ is carried by $\left\{\alpha \in \mathbf{R}, \tau_{\alpha} \in \Omega_{q}\right\}$, where $q$ is chosen as in $(2.8)$. 
3. General theory of processes over $\left(Y, Q_{m}\right)$. Let $m$ be an excessive measure. In this section we set down some of the "general theory of processes" for the stationary process $\left(Y, Q_{m}\right)$. Several of these results (or variants thereof) can be found in Dynkin [14] and Mitro [38]. See also [11] and [12] as general references.

We emphasize that $m \in$ Exc is fixed throughout this section. Various objects defined in the sequel depend on $m$ but this dependence is often suppressed from our notation.

Of particular importance is the "moderately Markovian" nature of $\left(Y_{t}\right)$ relative to the backward filtration $\left(\hat{\mathcal{G}}_{t}^{0}\right)$ defined by

$$
\hat{\mathcal{G}}_{t}^{0}=\sigma\left\{Y_{s}: s>t\right\}, \quad t \in \mathbf{R} .
$$

The $Q_{m}$-completion of $\left(\hat{\mathcal{G}}_{t}^{0}\right)$ is then $\hat{\mathcal{G}}_{t}^{m} \equiv \hat{\mathcal{G}}_{t}^{0} \vee \mathcal{N}^{m}$. Clearly both of these filtrations are decreasing and right continuous. Let $I^{m}$ denote the class of $Q_{m}$-evanescent processes on $\mathbf{R} \times W$ and set $\mathcal{M}^{0}=B \otimes \mathcal{G}^{0}, \mathcal{M}^{m}=\left(B \otimes \mathcal{G}^{m}\right) \vee I^{m}$. A monotone class argument shows that $\mathcal{M}^{m}=\mathcal{M}^{0} \vee I^{m}$. We define optional and "copredictable" $\sigma$-fields (uncompleted and completed) on $\mathbf{R} \times W$ as follows:

$$
\begin{aligned}
& 0^{0}=\sigma\left\{Z \in \mathcal{M}^{0}: Z_{t} \in \mathcal{G}_{t}^{0}, \forall t \in \mathbf{R} ; t \rightarrow Z_{t} \text { is rc; } Z=0 \text { on } \rrbracket-\infty, \alpha \llbracket\right\} ; \\
& 0^{m}=\sigma\left\{Z \in \mathcal{M}^{m}: Z_{t} \in \mathcal{G}_{t}^{m}, \forall t \in \mathbf{R} ; t \rightarrow Z_{t} \text { is rc a.e.- } Q_{m} ; Z=0 \text { on } \rrbracket-\infty, \alpha \llbracket\right\} ; \\
& \hat{p}^{0}=\sigma\left\{Z \in \mathcal{M}^{0}: Z_{t} \in \hat{\mathcal{G}}_{t}^{0}, \forall t \in \mathbf{R} ; t \rightarrow Z_{t} \text { is rc; } Z=0 \text { on } \llbracket \beta,+\infty \llbracket\right\} ; \\
& \hat{p}^{m}=\sigma\left\{Z \in \mathcal{M}^{m}: Z_{t} \in \hat{\mathcal{G}}_{t}^{m}, \forall t \in \mathbf{R} ; t \rightarrow Z_{t} \text { is rc a.e.- } Q_{m} ; Z=0 \text { on } \llbracket \beta,+\infty \llbracket\right\} .
\end{aligned}
$$

A random variable $T: W \rightarrow \mathbf{R} \cup\{-\infty,+\infty\}$ is an optional (resp. copredictable) time provided $\llbracket T,+\infty \llbracket \in \mathcal{O}^{0}$ (resp. $\left.\rrbracket-\infty, T \rrbracket \in \hat{P}^{0}\right)$. With $\mathcal{O}^{0}$ (resp. $\hat{p}^{0}$ ) replaced by $\mathcal{O}^{m}$ (resp. $\hat{\mathcal{P}}^{m}$ ) in these definitions, we obtain the notion of $Q_{m}$-optional (resp. $Q_{m}$-copredictable) time.

Parts (b) and (c) of the basic result to follow are the duals of known results on the killing operators $\left(k_{t}\right)$. See e.g., Azema-Jeulin [4]. We set

$$
\hat{\mathcal{G}}_{\alpha+}^{0}=\sigma\left\{A \cap\{\alpha<t\}: A \in \hat{\mathcal{G}}_{t}^{0}, t \in \mathbf{R}\right\} .
$$

(3.1) LemMA. (a) $0^{m}=0^{0} \vee I^{m}, \hat{p}^{m}=\hat{p}^{0} \vee I^{m}$.

(b) If $Z \in \mathcal{M}^{0}$ is constant on ] $-\infty, \alpha(w)$ ] for each $w$, then $Z \in \hat{p}^{0}$ if and only if $Z_{t}(w)=z\left(b_{t} w\right), \forall t, \forall w$, for some $z \in \hat{\mathcal{G}}_{\alpha+}^{0}$.

(c) If $Z \in \hat{p}^{0}$, then $Z_{t}=Z_{t} \circ b_{t}, \forall t \in \mathbf{R}$.

PROOF. (a) We consider only $\hat{p}^{m}$. Clearly $\hat{p}^{0} \vee I^{m} \subset \hat{p}^{m}$. For the reverse inclusion it suffices to show that each $Z \in\left(\mathcal{M}^{m}\right)^{+}$which is rc, adapted to $\left(\hat{\mathcal{G}}_{t}^{m}\right)$, and vanishing on $\llbracket \beta,+\infty \llbracket$, is in $\hat{p}^{0} \vee I^{m}$. Fix such a $Z$ and for $r \in \mathbf{Q}$ let $\bar{Z}_{r} \in \hat{\mathcal{G}}_{r}^{0}$ satisfy $Q_{m}\left(Z_{r} \neq \bar{Z}_{r}\right)=0$. Choose $B_{r} \in \hat{\mathcal{G}}_{r}^{0}$ with $B_{r} \subset\left\{Z_{r}=\bar{Z}_{r}\right\}$ and $Q_{m}\left(W \backslash B_{r}\right)=0$. Define $\tilde{Z} \in \hat{p}^{0}$ by

$$
\begin{aligned}
\tilde{Z}_{t}(w) & =\liminf _{r \downarrow t, r \in \mathbf{Q}} \bar{Z}_{r}(w) \quad \text { if } w \in \bigcap_{s>t, s \in \mathbf{Q}} B_{s}, t<\beta(w), \\
& =0 \quad \text { otherwise. }
\end{aligned}
$$

Evidently $Z$ and $\tilde{Z}$ are $Q_{m}$-indistinguishable. Thus $Z \in \hat{P}^{0} \vee I^{m}$. 
Point (c) is obvious since $b_{t}^{-1} \hat{\mathcal{G}}_{t}^{0}=\hat{\mathcal{G}}_{t}^{0}$ for all $t$. To prove the "only if" part of (b) it suffices to consider right continuous $Z$. Define $z \in \hat{\mathcal{G}}_{\alpha+}^{0}$ by

$$
z(w)=\liminf _{r \downarrow \alpha(w), r \in \mathbf{Q}} Z_{r}(w),
$$

with $z([\Delta])=0$. If $t \geq \beta(w)$ then $b_{t} w=[\Delta]$ and so $z\left(b_{t} w\right)=0=Z_{t}(w)$. If $t<\beta(w)$ then $\alpha\left(b_{t} w\right)=\alpha(w) \vee t$ and so, using (c) for the second equality below,

$$
\begin{aligned}
z\left(b_{t} w\right) & =\liminf _{r \downarrow \alpha(w) \vee t} Z_{r}\left(b_{t} w\right)=\liminf _{r \downarrow \alpha(w) \vee t} Z_{r}(w) \\
& =Z_{\alpha(w) \vee t}(w)=Z_{t}(w),
\end{aligned}
$$

where the final equality follows since $Z_{t}(w)$ is constant on ] $-\infty, \alpha(w)$ ]. The "if" part of (b) is easier and is left to the reader as an exercise.

The next result may be proved by the methods of Dynkin [14]. We omit a proof.

(3.2) Proposition. Let $\hat{\mathcal{K}}^{m}$ denote the class of processes $Z \in \mathcal{M}^{m}$ such that either $Z \vee 0$ or $(-Z) \vee 0$ is dominated by a constant process $(t, w) \rightarrow K(w)$, where $Q_{m}(K)<\infty$.

(a) If $Z \in \mathcal{K}^{m}$ then there exists a process ${ }^{\hat{p}} Z \in \hat{\mathcal{P}}^{m} \cap \mathcal{K}^{m}$ such that

$$
Q_{m}\left(Z_{T} ;-\infty<T<\beta\right)=Q_{m}\left({ }^{\hat{p}} Z_{T} ;-\infty<T<\beta\right)
$$

for all $Q_{m}$-copredictable times $T$.

(b) (SECTION THEOREM). If $Z^{i} \in \hat{P}^{m} \cap K^{m}(i=1,2)$ and

$$
Q_{m}\left(Z_{T}^{1} ;-\infty<T<\beta\right)=Q_{m}\left(Z_{T}^{2} ;-\infty<T<\beta\right)
$$

for all $Q_{m}$-copredictable times $T$, then $Z^{1}$ and $Z^{2}$ are $Q_{m}$-indistinguishable.

We refer to ${ }^{\hat{p}} Z$ as the $\left(Q_{m^{-}}\right)$copredictable projection of $Z$. The mapping $Z \rightarrow{ }^{\hat{p}} Z$ enjoys the usual properties of the predictable projection familiar in the general theory of processes (see $\left[\mathbf{1 2}\right.$, vol. II]). In particular, $Z \rightarrow{ }^{\hat{p}} Z$ is linear and preserves right continuity; $0 \leq{ }^{\hat{p}} Z \leq 1$ if $0 \leq Z \leq 1 \dot{p}^{\hat{p}}\left(Z^{n}\right) \uparrow^{\hat{p}} Z$ if $0 \leq Z^{n} \uparrow Z$. Of course, all of these statements hold modulo $I^{m}$.

The "big shifts" $\Sigma_{s}, s \in \mathbf{R}$, are defined by

$$
\Sigma_{s} Z_{t}=Z_{t-s} \circ \sigma_{s}, \quad t \in \mathbf{R}, Z \in \mathcal{M}^{m} .
$$

We say that $Z \in \mathcal{M}^{m}$ is homogeneous if $\Sigma_{s} Z=Z$, modulo $I^{m}$, for each $s \in \mathbf{R}$; if $\Sigma_{s} Z=Z$ holds indentically in $(s, w)$ then $Z$ is perfectly homogeneous. Note that since $Q_{m}$ is stationary, $\Sigma_{s}$ preserves the classes $I^{m}, O^{0}, \hat{p}^{0}, O^{m}, \hat{p}^{m}$, and that $\Sigma_{s}(\hat{p} Z)=\hat{p}\left(\Sigma_{s} Z\right)$, modulo $I^{m}$.

In discussing the moderately Markovian nature of $\left(Y_{t}\right)$ relative to $\left(\hat{\mathcal{G}}_{t}^{m}\right)$, some care must be taken with the birth time $\alpha$ (which is, in general, not copredictable). Such matters (relative to $\zeta$ in the context of right processes) have been clarified by Getoor and Sharpe in [29] and we follow their lead in defining a process $l$ by

$$
l=\hat{p}\left(1_{\rrbracket \alpha, \beta \llbracket) .}\right.
$$

Set $\Lambda=\{l>0\} \equiv\left\{(t, w) \in \mathbf{R} \times W: l_{t}(w)>0\right\}$. We can (and do) assume that a version of $l$ has been chosen such that $0 \leq l \leq 1$. Arguing as in [29] one sees that

$$
1_{\rrbracket \alpha, \beta \llbracket} \leq 1_{\Lambda} \leq 1_{\llbracket \alpha, \beta \llbracket} \quad\left(\text { modulo } I^{m}\right) .
$$


Later developments require that a "nice" version of $l$ be chosen. As a first step we have a

(3.4) LEMMA. Let $Z \in\left(O^{m}\right)^{+}$be $r c$, and vanishing on $\rrbracket-\infty, \alpha \rrbracket$. Then ${ }^{\hat{p}} Z \in \mathrm{O}^{m}$.

ProOF. Since $Z \rightarrow{ }^{\hat{p}} Z$ preserves right continuity, it suffices to show that ${ }^{\hat{p}} Z_{t} \in$ $\mathcal{G}_{t}^{m}$ for all $t \in \mathbf{R}$. Clearly ${ }^{\hat{p}} Z=0$ on $\llbracket \beta,+\infty \llbracket$ and also on $\rrbracket-\infty, \alpha \llbracket$ (since $Z=0$ on $\rrbracket-\infty, \alpha \rrbracket)$. Fix $t \in \mathbf{R}$ and $A \in \hat{\mathcal{G}}_{t}^{m}$. Using the $\sigma$-finiteness of $Q_{m}$ on $\mathcal{G}_{t}^{m} \cap\{\alpha<t<$ $\beta$ \} we conclude that the conditional expectation $Q_{m}\left(A \mid \mathcal{G}_{t}^{m}\right)$ (of $A \cap\{\alpha<t<\beta\}$ given $\left.\mathcal{G}_{t}^{m} \cap\{\alpha<t<\beta\}\right)$ is measurable over $\sigma\left(Y_{t}\right) \cap\{\alpha<t<\beta\} \subset \hat{\mathcal{G}}_{t}^{m} \cap\{\alpha<t<\beta\}$. Thus, using $Q_{m}(\alpha=t)=0$, we compute

$$
\begin{aligned}
Q_{m}\left(\hat{p} Z_{t} ; A \cap\{t<\beta\}\right) & =Q_{m}\left(Z_{t} ; A \cap\{t<\beta\}\right) \\
& =Q_{m}\left(Z_{t} ; A \cap\{\alpha<t<\beta\}\right) \\
& =Q_{m}\left(Z_{t} Q_{m}\left(A \mid \mathcal{G}_{t}^{m}\right) ; \alpha<t<\beta\right) \\
& =Q_{m}\left({ }^{\hat{p}} Z_{t} Q_{m}\left(A \mid \mathcal{G}_{t}^{m}\right) ; t<\beta\right) .
\end{aligned}
$$

Since $A \in \hat{\mathcal{G}}_{t}^{m}$ was arbitrary, and since $\mathcal{G}_{t}^{m} \vee \hat{\mathcal{G}}_{t}^{m}=\mathcal{G}^{m}$, we may conclude that ${ }^{\hat{p}} Z_{t} \in \mathcal{G}_{t}^{m}$ as desired.

(3.5) Proposition. There exists a function $f \in \mathcal{G}_{0+}^{0} \cap \hat{\mathcal{G}}_{0}^{0}$ with $0 \leq f \leq 1$, such that $l$ and $\left(f\left(\sigma_{t}\right)\right)_{t \in \mathbf{R}}$ are $Q_{m}$-indistinguishable. In particular, $l \in \mathcal{O}^{m}$.

ProOF. By a construction given in [19] there exists a sequence $\left(S_{n}\right)$ of $\left(\mathcal{G}_{t+}^{0}\right)$ stopping times such that $\alpha \leq S_{n}<\beta$ on $\left\{S_{n}<+\infty\right\}, t+S_{n} \circ \sigma_{t}=S_{n}, \forall t \in \mathbf{R}$, and

(i) $S_{n}=-\infty$ on $\{\alpha=-\infty\}$,

(ii) $S_{n}>\alpha, S_{n} \downarrow \alpha$ as $n \rightarrow \infty$, a.s. $Q_{m}$ on $\{\alpha>-\infty\}$.

Let $Z^{n}=1_{\llbracket S_{n}, \beta \llbracket}$ so that $Z^{n} \in \mathcal{O}^{0}, Z^{n}$ is rc and perfectly homogeneous. Clearly $Z^{n} \uparrow 1_{\rrbracket \alpha, \beta \llbracket}$ and so ${ }^{\hat{p}}\left(Z^{n}\right) \uparrow l$, modulo $I^{m}$, as $n \uparrow \infty$. By $(3.4),{ }^{\hat{p}}\left(Z^{n}\right)$ is $O^{m}$. measurable. Moreover, ${ }^{\hat{p}}\left(Z^{n}\right)$ is homogeneous and right continuous. By a result of Benveniste [5, p. $100 \mathrm{ff}$.] there is an $f_{n} \in \mathcal{G}_{0+}^{0}$ with $0 \leq f_{n} \leq 1$, such that $\hat{p}\left(Z_{n}\right)$ is $Q_{m}$-indistinguishable from $\left(f_{n}\left(\sigma_{t}\right)\right)_{t \in \mathbf{R}}$. By (3.1a) we may assume that $\hat{p}\left(Z^{n}\right) \in \hat{p}^{0}$ and then the argument used in [5] shows that $f_{n}$ may be chosen to satisfy $f_{n}=f_{n} \circ b_{0}$; that is, $f_{n} \in \hat{\mathcal{G}}_{0}^{0}$. Taking $f=\limsup _{n \rightarrow \infty} f_{n}$ we have $0 \leq f \leq 1, f \in \mathcal{G}_{0+}^{0} \cap \hat{\mathcal{G}}_{0}^{0}$ and $l=f \circ \sigma$, modulo $I^{m}$, as required.

Because of (3.5) we can, and do, assume that a perfectly homogeneous, $0^{0} \cap \hat{p}^{0}$. measurable version of $l$ has been chosen; the set $\Lambda=\{l>0\}$ then has the same properties. A further refinement of $l$ results from the next lemma. Recall the definition of $\Omega_{q}$ given prior to (2.8), where $q \in \mathcal{E}^{+}$satisfies $q>0$ and $m(q)<\infty$.

(3.6) Lemma. Modulo $I^{m}, \Lambda \subset\left\{\tau . \in \Omega_{q}\right\} \equiv\left\{(t, w) \in \mathbf{R} \times W: \tau_{t} w \in \Omega_{q}\right\}$.

PROOF. Because $\left(P_{s}\right)$ is a Borel right semigroup, it is clear that modulo $I^{m}$,

$$
1_{\rrbracket \alpha, \beta \llbracket} \leq 1_{\rrbracket \alpha, \beta \llbracket} 1_{\left\{r, \in \Omega_{q}\right\}} .
$$

Taking $Q_{m}$-copredictable projections in (3.7) we obtain

$$
l \leq l 1_{\{\tau \in \Omega q\}}, \quad \text { modulo } I^{m},
$$

and the desired inclusion follows. 
Thus, replacing $l$ (resp. $\Lambda$ ) by $l 1_{\left\{\tau \in \Omega_{q}\right\}}$ (resp. $\Lambda \cap\left\{\tau . \in \Omega_{q}\right\}$ ) we may assume that the inclusion asserted in (3.6) holds with no exceptional points. Of course, since $\tau_{t} \circ \sigma_{s}=\tau_{t+s}$ and since $\left\{\tau . \in \Omega_{q}\right\} \in 0^{0} \cap \hat{P}^{0}$, the modified choices of $l$ and $\Lambda$ are perfectly homogeneous and $0^{0} \cap \hat{P}^{0}$-measurable.

Previous authors (e.g., $[\mathbf{1 4}, \mathbf{3 8}])$ have defined an "optional projection" over $\left(Y, Q_{m}\right)$ but these projections are not adequate for our needs since they vanish off $\rrbracket \alpha, \beta \llbracket$. We shall obtain an extended optional projection, defined on $\Lambda$, that will be adequate when considering random measures carried by $\Lambda$. To this end we introduce an extension $\left(\bar{Y}_{t}\right)$ of the basic process

$$
\begin{aligned}
\bar{Y}_{t}(w) & =Y_{t}(w), \quad t \neq \alpha(w) \text { or } t=\alpha(w)>-\infty \text { and }(t, w) \notin \Lambda ; \\
& =Y_{t+}(w), \quad t=\alpha(w)>-\infty, .(t, w) \in \Lambda .
\end{aligned}
$$

Note that $Y_{t+}(w)$ exists in $E$ if $t=\alpha(w)>-\infty$ and $(t, w) \in \Lambda$ since $\tau_{t} w \in \Omega_{q}$ in this case. In particular,

$$
\Lambda=\left\{(t, w) \in \mathbf{R} \times W: \bar{Y}_{t}(w) \in E\right\} .
$$

Moreover, $\bar{Y}=\left(\bar{Y}_{t}\right)_{t \in \mathbf{R}}$ is perfectly homogeneous, $0^{0} \cap \hat{p}^{0}$-measurable, and rc on $\Lambda$. Note also that if $f \in \mathcal{E}^{+}$or $b \mathcal{E}$, then

$$
\hat{p}\left(f \circ Y_{t}\right)=f\left(\bar{Y}_{t}\right) l_{t}, \quad \text { modulo } I^{m} .
$$

Indeed, if $T$ is a $Q_{m}$-copredictable time,

$$
\begin{aligned}
Q_{m}\left(\hat{p}(f \circ Y)_{T} ;-\infty<T<\beta\right) & =Q_{m}\left(f \circ Y_{T} ;-\infty<T<\beta\right) \\
& =Q_{m}\left(f \circ Y_{T} 1_{\rrbracket \alpha, \beta \llbracket}(T) ;-\infty<T<\beta\right) \\
& =Q_{m}\left(f \circ \bar{Y}_{T} 1_{\rrbracket \alpha, \beta \llbracket}(T) ;-\infty<T<\beta\right) \\
& =Q_{m}\left(f \circ \bar{Y}_{T} l_{T} ;-\infty<T<\beta\right),
\end{aligned}
$$

where for the third equality we have used the fact that $Y=\bar{Y}$ on $\rrbracket \alpha, \beta \llbracket$.

The key point in constructing our projection is the following strong Markov property of $\bar{Y}$, extending (2.3).

(3.10) Proposition. Let $T$ be a $Q_{m}$-optional time and let $F \in\left(\mathcal{F}^{0}\right)^{+}$. Then $Q_{m}$ is $\sigma$-finite on $\mathcal{G}_{T}^{m} \cap\{T \in \Lambda\}$ and

$$
Q_{m}\left(F \circ \tau_{T} ; T \in \Lambda\right)=Q_{m}\left(P^{\bar{Y}_{T}}(F) ; T \in \Lambda\right)
$$

ProOF. In proving (3.11) we may assume that $T$ is optional (i.e., $\llbracket T,+\infty \llbracket \in$ $\left.0^{0}\right)$. Also, since $Y=\bar{Y}$ on $\rrbracket \alpha, \beta \llbracket$, because of $(2.3)$ it suffices to show

$$
Q_{m}\left(F \circ \tau_{T} ; T \in \Gamma\right)=Q_{m}\left(P^{\bar{Y}_{T}}(F) ; T \in \Gamma\right),
$$

where $\Gamma=\Lambda \backslash \rrbracket \alpha, \beta \llbracket \subset \llbracket \alpha \rrbracket$. Let $\nu=\left(\nu_{t}\right)$ denote the entrance law such that $m_{p}$, the purely excessive part of $m$, is given by $m_{p}=\int_{0}^{\infty} \nu_{t} d t$. Let $Q_{\nu}$ denote the corresponding Kuznetsov measure. Since $\left\{l_{0}>0\right\} \in \mathcal{G}_{0+}^{0}$, it is easy to check that $Q_{\nu}\left(\cdot ; l_{0}>0\right)$ is the Kuznetsov measure for the entrance law $\tilde{\nu}$ given by

$$
\tilde{\nu}_{t}(f)=Q_{\nu}\left(f \circ Y_{t} ; l_{0}>0\right) .
$$


From (3.6) and (2.2b) we see that $Q_{\tilde{\nu}}$ is carried by $\Omega_{q}$. It follows from (2.8) that $Q_{\tilde{\nu}}=P^{\rho}$, where $\rho(f)=Q_{\tilde{\nu}}\left(f \circ Y_{0+}\right)=Q_{\nu}\left(f \circ Y_{0+} ; l_{0}>0\right)$. Define

$$
T_{t}(w)=\left[T\left(b_{t} \sigma_{-t} w\right)-t\right] \vee 0
$$

Clearly $(t, w) \rightarrow T_{t}(w)$ is $B \otimes \mathcal{G}^{0}$-measurable and $\left\{T_{t} \leq u\right\} \in \mathcal{G}_{u+}^{0}$ if $u \geq 0$. Thus $T_{t} \mid \Omega$ is an $\left(\mathcal{F}_{u+}^{0}\right)$-stopping time. Also, $b_{\alpha} w=w$ if $\alpha(w) \in \mathbf{R}$; thus

$$
T_{\alpha(w)}\left(\tau_{\alpha}(w)\right)=T(w)-\alpha(w) \quad \text { if } \alpha(w) \in \mathbf{R} .
$$

Let $g \geq 0$ be Lebesgue integrable over $\mathbf{R}$. Using (2.5) we compute

$$
\begin{aligned}
Q_{m}\left(g(T) F \circ \tau_{T} ; T \in \Gamma\right) & =Q_{m}\left(g(\alpha) F \circ \tau_{\alpha} ; T=\alpha, l_{\alpha}>0, \alpha \in \mathbf{R}\right) \\
& =Q_{m}\left(g(\alpha) F \circ \tau_{\alpha} ; T_{\alpha}\left(\tau_{\alpha}\right)=0, l_{0} \circ \tau_{\alpha}>0, \alpha \in \mathbf{R}\right) \\
& =\int_{\mathbf{R}} g(t) Q_{\nu}\left(F ; T_{t}=0, l_{0}>0\right) d t \\
& =\int_{\mathbf{R}} g(t) Q_{\nu}\left(P^{Y_{0}}(F) ; T_{t}=0, l_{0}>0\right) d t \\
& =Q_{m}\left(g(\alpha) P^{Y_{\alpha}}(F) ; T=\alpha, l_{\alpha}>0, \alpha \in \mathbf{R}\right) \\
& =Q_{m}\left(g(T) P^{\bar{Y}_{T}}(F) ; T \in \Gamma\right) .
\end{aligned}
$$

We have used the 0-1 law and the fact that $Q_{\nu}\left(\cdot ; l_{0}>0\right)=P^{\rho}$ in the fourth equality above. Letting $g \uparrow 1$ through a sequence we obtain (3.12), whence (3.11). Now $Q_{m}$ is $\sigma$-finite on $\mathcal{G}_{T}^{m} \cap\{\alpha<T<\beta\}$, as was mentioned following (2.3). Since $\rho$ is $\sigma$-finite we may choose $f \in \mathcal{E}^{+}$with $f>0$ and $\rho(f)<\infty$; let $g>0$ be Lebesgue integrable over R. Then $g(T) f\left(\bar{Y}_{T}\right)>0$ on $\{T \in \Gamma\}$ and by the previous computation

$$
Q_{m}\left(g(T) f\left(\bar{Y}_{T}\right) ; T \in \Gamma\right) \leq \int_{\mathbf{R}} g(t) P^{\rho}\left(f \circ Y_{0+}\right) d t=\lambda(g) \rho(f)<\infty .
$$

(Here $\lambda$ denotes Lebesgue measure.) Thus $Q_{m}$ is $\sigma$-finite on $\mathcal{G}_{T}^{m} \cap\{T \in \Gamma\}$ and so is $\sigma$-finite on $\mathcal{G}_{T}^{m} \cap\{T \in \Lambda\}$ as claimed.

A monotone class argument yields the following standard extension of (3.11). Let $T$ be $Q_{m}$-optional, $F \in\left(\mathcal{G}_{T}^{m} \otimes \mathcal{G}^{0}\right)^{+}$. Then

$$
\int_{\{T \in \Lambda\}} F\left(w, \tau_{T} w\right) Q_{m}(d w)=\int_{\{T \in \Lambda\}} \int_{\Omega} F\left(w, w^{\prime}\right) P^{\bar{Y}_{T(w)}}\left(d w^{\prime}\right) Q_{m}(d w) .
$$

Define a "splicing map" $\left(w / t / w^{\prime}\right)$ as follows: $\left(w / t / w^{\prime}\right)$ is the element of $W$ satisfying

$$
\begin{aligned}
\left(w / t / w^{\prime}\right)(u)= & w_{u+}^{\prime}, u \geq t \\
& w_{u}, u<t \\
= & b_{t} w^{\prime} \\
= & \Delta
\end{aligned}
$$$$
\text { if }\left(t, w^{\prime}\right) \in \Lambda, \alpha(w)<t<\beta(w),
$$$$
\text { if }\left(t, w^{\prime}\right) \in \Lambda, t \leq \alpha(w) \text {, }
$$
otherwise.

One checks that if $(t, w) \in \Lambda$, then $w=(w / t / w)=\left(k_{t} w / t / b_{t} w\right)$. Thus given a process $Z=\left(Z_{t}\right)$, if we set $H\left(w, t, w^{\prime}\right)=Z_{t}\left(w / t / \sigma_{-t} w^{\prime}\right)$, then

$$
Z_{t}(w)=H\left(k_{t} w, t, \tau_{t} w\right), \quad(t, w) \in \Lambda .
$$

Now given $Z \in\left(\mathcal{M}^{m}\right)^{+}$choose $\tilde{Z} \in \mathcal{M}^{0}$ such that $Z$ and $\tilde{Z}$ are $Q_{m}$-indistinguishable. Define a process ${ }^{\circ} Z$ by

$$
{ }^{\circ} Z_{t}(w)=1_{\Lambda}(t, w) \int_{\Omega} \tilde{Z}_{t}\left(w / t / \sigma_{-t} w^{\prime}\right) P^{\bar{Y}_{t}(w)}\left(d w^{\prime}\right) .
$$

Since $\tilde{Z} \in \mathcal{M}^{0}$, the right side of $(3.15)$ is $0^{0}$-measurable in $(t, w)$. 
(3.16) Theorem. (a) Given $Z \in\left(\mathcal{M}^{m}\right)^{+}$define ${ }^{\circ} Z$ as in (3.15). If $T$ is any $Q_{m}$-optional time, then

$$
Q_{m}\left(Z_{T} ; T \in \Lambda\right)=Q_{m}\left({ }^{\circ} Z_{T} ; T \in \Lambda\right) .
$$

(b) If $Z^{1}, Z^{2}$ are positive, $0^{m}$-measurable processes and if $Q_{m}\left(Z_{T}^{1} ; T \in \Lambda\right)$ $=Q_{m}\left(Z_{T}^{2} ; T \in \Lambda\right)$ for all $Q_{m}$-optional times $T$, then $Z^{1} 1_{\Lambda}$ and $Z^{2} 1_{\Lambda}$ are $Q_{m^{-}}$ indistinguishable.

PROOF. (a) This follows immediately from (3.13) since

$$
\left(w, w^{\prime}\right) \rightarrow \tilde{H}\left(w, T(w), w^{\prime}\right)
$$

is $\mathcal{G}_{T+}^{0} \otimes \mathcal{G}^{0}$-measurable, where $\tilde{H}\left(w, t, w^{\prime}\right)=\tilde{Z}_{t}\left(w / t / \sigma_{-t} w^{\prime}\right)$.

(b) This assertion can be reduced to the usual optional section theorem. We prove a similar point below (Proposition (3.19)) and so omit a proof.

(3.18) REMARKS. (a) The construction of an optional projection by means of the "Dawson formula" (3.15) is standard in Markov process theory. A similar construction of optional projection on $\rrbracket \alpha, \beta \llbracket$ can be found in [38].

(b) We shall refer to ${ }^{\circ} Z$ as the $Q_{m}$-optional projection of $Z$. The mapping $Z \rightarrow{ }^{\circ} Z$ has properties analogous to those listed earlier for ${ }^{\hat{p}} Z$. One fact not immediately evident is the preservation of right continuity detailed in the next result.

(3.19) Proposition. Suppose that $Z \in b K^{m}$ is right continuous a.s. $Q_{m}$. Then ${ }^{\circ} Z$ is right continuous on $\Lambda$ a.s. $Q_{m}$.

ProOF. Fix a $Q_{m}$-optional time $T$ with $Q_{m}(T \in \Lambda)>0$. Let $F \in\left(\mathcal{G}_{T}^{m}\right)^{+}$with $\{F>0\}=\{T \in \Lambda\}$ and $Q_{m}(F)<\infty$ (such an $F$ exists since $Q_{m}$ is $\sigma$-finite on $\mathcal{G}_{T}^{m} \cap\{T \in \Lambda\}$ by (3.12)). Define a finite measure $\Pi$ by $\Pi(G)=Q_{m}(F G), G \in \mathcal{G}^{m}$. Let $\left(\mathscr{H}_{t}\right)_{t \geq 0}$ denote the right continuous completion of $\left(\mathcal{G}_{T+t}^{m}\right)_{t \geq 0}$ relative to $\Pi$. Define processes $B$ and $\tilde{B}$ by $B_{t}=Z_{T+t}, \tilde{B}_{t}={ }^{\circ} Z_{T+t}$ and note that $B$ is rc on $[0,+\infty[$. Let $C$ denote the optional projection of $B$ relative to the system $\left(W,\left(\mathscr{H}_{t}\right), \Pi\right)$. It is standard (see $\left.[12, \mathrm{VI} .2, \mathrm{~T} 47]\right)$ that $C$ is rc on $[0,+\infty[$ a.s. $\Pi$. We claim that $C$ and $\tilde{B}$ are $\Pi$-indistinguishable (as processes with parameter set $\left[0,+\infty[)\right.$. To see this let $S \geq 0$ be a stopping time of $\left(H_{t}\right)$ and choose a stopping time $S^{\prime} \geq 0$ of $\left(\mathcal{G}_{T+t}^{m}\right)_{t \geq 0}$ such that $\Pi\left(S \neq S^{\prime}\right)=0$. Then $T+S^{\prime}$ is a $Q_{m}$-optional time and so, applying (3.17) at time $T+S^{\prime}$, we have

$$
\begin{aligned}
\Pi\left(C_{S} ; S<\infty\right) & =\Pi\left(B_{S} ; S<\infty\right) \\
& =Q_{m}\left(Z_{T+S^{\prime}} \cdot F ; T \in \Lambda, S^{\prime}<\infty\right) \\
& =Q_{m}\left({ }^{\circ} Z_{T+S^{\prime}} \cdot F ; T \in \Lambda, S^{\prime}<\infty\right) \\
& =\Pi\left(\tilde{B}_{S} ; S<\infty\right) .
\end{aligned}
$$

Evidently $\tilde{B}$ is $\left(H_{t}\right)$-optional and so $C$ and $\tilde{B}$ are $\Pi$-indistinguishable by the section theorem. It follows that ${ }^{\circ} Z_{T+t}$ is rc in $t \geq 0$ a.s. $Q_{m}$ on $\{T \in \Lambda\}$. Since $T$ was arbitrary, the right continuity of ${ }^{\circ} Z$ follows.

We close this section with several applications of the preceding results that will be useful in the sequel. 
(3.20) Proposition. Let $Z \geq 0$ be $0^{m} \cap \hat{P}^{m}$-measurable. Then $Z 1_{\Lambda}$ is $Q_{m}$ indistinguishable from a process of the form $f\left(t, \bar{Y}_{t}\right)$ where $f \in(B \otimes \mathcal{E})^{+}$(and $f(t, \Delta)=0$ by convention $)$.

Proof. By (3.1) we may assume that $Z \in\left(\mathrm{O}^{m} \cap \hat{P}^{0}\right)^{+}$and then $Z_{t} \circ b_{t}=Z_{t}$ identically in $(t, w)$. Let $H_{t}=Z_{t} \circ \sigma_{-t}$ so that $Z_{t}=Z_{t} \circ b_{t}=H_{t} \circ \tau_{t}$. Let $f(t, x)=P^{x}\left(H_{t}\right)$ if $(t, x) \in \mathbf{R} \times E$ and set $f(t, \Delta)=0$. Then $f \in(B \otimes \mathcal{E})^{+}$and $\left(w, w^{\prime}\right) \rightarrow H_{T(w)}\left(w^{\prime}\right)$ is $\mathcal{G}_{T}^{m} \otimes \mathcal{G}^{0}$-measurable. Thus, using (3.13),

$$
\begin{aligned}
Q_{m}\left(Z_{T} ; T \in \Lambda\right) & =Q_{m}\left(H_{T}\left(\tau_{T}\right) ; T \in \Lambda\right) \\
& =\int_{\{T \in \Lambda\}} \int_{\Omega} H_{T(w)}\left(w^{\prime}\right) P^{\bar{Y}_{T(w)}}\left(d w^{\prime}\right) Q_{m}(d w) \\
& =Q_{m}\left(f\left(T, \bar{Y}_{T}\right) ; T \in \Lambda\right) .
\end{aligned}
$$

Since $Z \in O^{m}$, the section theorem (3.16) allows us to conclude that $Z 1_{\Lambda}=f(\cdot, \bar{Y}$.) modulo $I^{m}$.

(3.21) COROLLARY. If $Z \in\left(\mathrm{O}^{m} \cap \hat{p}^{m}\right)^{+}$is homogeneous, then there is a function $f \in \mathcal{E}^{+}$such that $Z 1_{\Lambda}=f\left(\bar{Y}\right.$.) modulo $I^{m}$.

PROOF. We may assume that $Z=0$ off $\Lambda$ and that $Z_{t}=g\left(t, \bar{Y}_{t}\right)$ where $g \in$ $(B \otimes \mathcal{E})^{+}$, because of $(3.20)$. Since $Z$ is homogeneous, for each $s \in \mathbf{R}$ we have

$$
g\left(t-s, \bar{Y}_{t}\right)=g\left(t, \bar{Y}_{t}\right), \quad \forall t \in \mathbf{R}, \quad \text { a.s. } Q_{m} .
$$

By Fubini's theorem for $Q_{m}$-a.e. $w \in W$ there is a Lebesgue null set $N_{w} \subset \mathbf{R}$ such that for $s \notin N_{w}, g\left(t-s, \bar{Y}_{t}(w)\right)=g\left(t, \bar{Y}_{t}(w)\right), \forall t \in \mathbf{R}$. Set

$$
f(x)=\int_{0}^{1} g(u, x) d u, \quad x \in E .
$$

Clearly $f \in \mathcal{E}^{+}$and for $Q_{m}$-a.e. $w$, for all $t \in \mathbf{R}$,

$$
\begin{aligned}
Z_{t}(w) & =g\left(t, \bar{Y}_{t}(w)\right)=\int_{t-1}^{t} g\left(t-s, \bar{Y}_{t}(w)\right) d s \\
& =f\left(\bar{Y}_{t}(w)\right) . \quad \square
\end{aligned}
$$

Recall from [29] that a Borel set $B \subset E$ is m-polar provided $Q_{m}\left(Y_{t} \in B\right.$, some $t \in \mathbf{R})=0$. Evidently $B \in \mathcal{E}$ is $m$-polar if and only if $P^{m}\left(X_{s} \in B\right.$, some $\left.s>0\right)=0$. Because of the following result we may conclude that the function $f$ in (3.21) is uniquely determined up to an $m$-polar set.

(3.22) Proposition. A Borel set $B \subset E$ is $m$-polar if and only if

$$
Q_{m}\left(\bar{Y}_{t} \in B \text {, some } t \in \mathbf{R}\right)=0 \text {. }
$$

Proof. Since $Y=\bar{Y}$ on $\rrbracket \alpha, \beta \llbracket$, and since $Y=\Delta$ off $\rrbracket \alpha, \beta \llbracket$, it is clear that if (3.23) holds then $B$ is $m$-polar. Conversely, if $B$ is $m$-polar then the process $Z_{t}=1_{B}\left(Y_{t}\right)$ is $Q_{m}$-evanescent. By (3.9), the $Q_{m}$-evanescent process ${ }^{\hat{p}} Z$ is $Q_{m^{-}}$ indistinguishable from $1_{B}\left(\bar{Y}_{t}\right) l_{t}$. Since $\bar{Y}=\Delta$ off $\Lambda=\{l>0\}$, we conclude that $1_{B}\left(\bar{Y}_{t}\right)$ is evanescent and so $(3.23)$ holds. 
4. The moderate Markov dual process. The simple Markov property of $\left(Y, Q_{m}\right)$ being symmetric with respect to past and future, it is clear that $\left(Y_{t}, \hat{\mathcal{G}}_{t}^{m}\right.$, $\left.Q_{m}\right)$ is a simple Markov process. The basic work of Chung and Walsh [10], on the time reversal of strong Markov processes, indicates that even more is true: the modified process $\left(\bar{Y}_{t}\right)$ is "moderately" Markov relative to $\left(\hat{\mathcal{G}}_{t}^{m}\right)$. This fact, detailed in Theorem (4.6) below, is an extension of similar results proved by various authors $[\mathbf{2}, \mathbf{1 0}, \mathbf{2 0}, \mathbf{3 2}, \mathbf{3 3}, \mathbf{4 0}]$. None of these papers quite covers the present circumstances (in [40 and 20] Meyer's hypothesis $(L)$ is imposed; the other papers cover only the case $m \in$ Pot). Our argument, which follows closely that of Azema [2] (see also [32]), is deferred to an appendix. In this section, after stating the basic result (4.6), we detail some of its consequences to be used in the sequel.

We begin with some notation. Throughout this section $m \in$ Exc is fixed. Set $\hat{\Omega}=\{w \in W: \beta(w)=0\} \cup\{[\Delta]\}, \hat{\mathcal{F}}^{0}=\mathcal{G}^{0} \cap \hat{\Omega}$ and define a process $\hat{X}$ by $\hat{X}_{s}=$ $\bar{Y}_{-s}, s>0$ (on $\hat{\Omega}$ ). We should emphasize that $\hat{X}_{s}$ depends on $m$ through $l$ and $\Lambda$ and is not the same as the "coordinate" process $s \rightarrow w_{-s}$ on $\hat{\Omega}$. Nonetheless we do have $\hat{\mathcal{F}}^{0}=\sigma\left\{\hat{X}_{s}: s>0\right\}$, as is easily deduced. We define shift operators dual to $\tau_{t}$ and $\theta_{s}$ by

$$
\begin{aligned}
& \hat{\tau}_{t}=k_{0} \sigma_{t}=\sigma_{t} k_{t}, \quad t \in \mathbf{R} ; \\
& \hat{\theta}_{s}=\hat{\tau}_{-s}, \quad s>0 \quad(\text { on } \hat{\Omega}) .
\end{aligned}
$$

Note that if $s<t$ then $\hat{X}_{t-s} \circ \hat{\tau}_{t}=\bar{Y}_{s}$ on $\{t<\beta\}$. Clearly the lifetime of $\hat{X}$ is given by $\hat{\zeta}=(-\alpha) \vee 0$. Finally, let $\hat{\mathcal{F}}_{t}^{0}=\sigma\left\{\hat{X}_{s}: 0<s \leq t\right\}$ and let $P\left(\hat{\mathcal{F}}_{t}^{0}\right)$ denote the class of $\left(\hat{\mathcal{F}}_{t}^{0}\right)$-predictable processes (with parameter set $] 0,+\infty[$ ). Note that $\hat{X}$ is predictable in that $f\left(\hat{X}_{\text {. }}\right) \in \mathcal{P}\left(\hat{\mathcal{F}}_{t}^{0}\right)$ if $f \in b \mathcal{E}$ or $\mathcal{E}^{+}$.

(4.1) Definition. Let $\left\{\hat{P}^{x}: x \in E_{\Delta}\right\}$ be a Markov kernel from $\left(E_{\Delta}, \mathcal{E}_{\Delta}\right)$ to $\left(\hat{\Omega}, \hat{\xi}^{0}\right)$ with $\hat{P}^{\Delta}=\varepsilon_{[\Delta]}$. We say that $\left(\hat{P}^{x}\right)$ is a moderate Markov dual process for $\left(P^{x}\right)$ relative to $m$, provided

$$
Q_{m}\left(F \circ \hat{\tau}_{T} ;-\infty<T<\beta\right)=Q_{m}\left(\hat{P}^{\bar{Y}_{T}}(F) ;-\infty<T<\beta\right)
$$

for all $F \in\left(\hat{\mathcal{F}}^{0}\right)^{+}$and all $Q_{m}$-copredictable times $T$, and provided

(4.3) for each $x \in E, \hat{X}$ under $\hat{P}^{x}$ is moderately Markov relative to $\left(\hat{P}^{\cdot}\right)$ : for all $F \in\left(\hat{\mathcal{F}}^{0}\right)^{+}$and all $\left(\hat{\mathcal{F}}_{t}^{0}\right)$-predictable times $T$,

$$
\hat{P}^{x}\left(F \circ \hat{\theta}_{T} ; 0<T<+\infty\right)=\hat{P}^{x}\left(\hat{P}^{\hat{X}_{T}}(F) ; 0<T<\infty\right) .
$$

(4.5) REMARK. Let us write

$$
\begin{gathered}
\hat{l}_{t}(w)=l_{-t}(w) \quad \text { for } t>0, w \in \hat{\Omega} \\
\hat{\Lambda}=\{(t, w) \in] 0,+\infty\left[\times \hat{\Omega}: \hat{l}_{t}(w)>0\right\} .
\end{gathered}
$$

Clearly $\hat{X}$ is lc on $\hat{\Lambda}$ although not necessarily on $] 0,+\infty[$. Thus (4.4) differs slightly from the usual moderate Markov property $[\mathbf{9}, \mathbf{4 0}]$ which requires an lc process.

Here is our fundamental result concerning a dual process for $X$. As mentioned previously its proof is deferred to an appendix.

(4.6) THEOREM. There exists a kernel $\left\{\hat{P}^{x}: x \in E_{\Delta}\right\}$ such that (4.1) is valid. If $\left\{\tilde{P}^{x}: x \in E_{\Delta}\right\}$ is a second kernel satisfying (4.2), then $\left\{x: \hat{P}^{x} \neq \tilde{P}^{x}\right\}$ is a Borel $m$-polar set. 
For the remainder of this section we fix a kernel $\left\{\hat{P}^{x}: x \in E_{\Delta}\right\}$ satisfying (4.1). Firstly, we define a semigroup $\left(\hat{P}_{t}\right)$ by

$$
\hat{P}_{t} f(x)=\hat{P}^{x}\left(f \circ \hat{X}_{t}\right), \quad t>0, f \in b \mathcal{E} \text { or } \mathcal{E}^{+} .
$$

The semigroup property of $\left(\hat{P}_{t}\right)$ follows upon taking $T=t$ and $F=f \circ \hat{X}_{s}$ in (4.4). Next, let $f, g \in \mathcal{E}^{+}$and $t>0$. Since $Q_{m}(\alpha=t)=0$ we have $Q_{m}(t \in \Lambda \backslash \rrbracket \alpha, \beta \llbracket)=0$; using (4.2) we compute

$$
\begin{aligned}
m\left(g \cdot P_{t} f\right) & =Q_{m}\left(g \circ Y_{0} P_{t} f \circ Y_{0}\right)=Q_{m}\left(g \circ Y_{0} f \circ \bar{Y}_{t} ; t<\beta\right) \\
& =Q_{m}\left(\hat{P}_{t} g \circ \bar{Y}_{t} f \circ \bar{Y}_{t} ; t<\beta\right)=m\left(\hat{P}_{t} g \cdot f\right) .
\end{aligned}
$$

That is, $\left(P_{t}\right)$ and $\left(\hat{P}_{t}\right)$ are in duality relative to $m$.

As a first application of the dual family $\left(\hat{P}^{x}\right)$ we prove the commutation relation ${ }^{\circ \hat{p}} Z={ }^{\hat{p} \circ} Z$ on $\Lambda$ (modulo $I^{m}$ ). This is accomplished by providing an explicit formula for ${ }^{\hat{p}} Z$ when $Z \in \mathcal{M}^{0}$; this formula is "dual" to (3.15).

Given $Z \in \mathcal{M}^{0}$ define $H$ by

$$
H\left(w, t, w^{\prime}\right)=Z_{t}\left(\sigma_{-t} w / t / \sigma_{-t} w^{\prime}\right)
$$

(and note that this definition differs from that of the last section). As before

$$
Z_{t}(w)=H_{t}\left(\hat{\tau}_{t} w, t, \tau_{t} w\right) \quad \text { if }(t, w) \in \Lambda .
$$

Translating (3.15) into the present notation, a version of the option projection of $Z$ is given by

$$
{ }^{\circ} Z(t, w)=1_{\Lambda}(t, w) \int_{\Omega} H\left(\hat{\tau}_{t} w, t, w^{\prime}\right) P^{\bar{Y}_{t}(w)}\left(d w^{\prime}\right)
$$

(4.10) Proposition. If $Z \in\left(\mathcal{M}^{0}\right)^{+}$, then a version of ${ }^{\hat{p}} Z 1_{\Lambda}$ is given by

$$
(t, w) \rightarrow 1_{\Lambda}(t, w) \int_{\hat{\Omega}} H\left(w^{\prime \prime}, t, \tau_{t} w\right) \hat{P}^{\bar{Y}_{t}(w)}\left(d w^{\prime \prime}\right) .
$$

PROOF. This result follows from (4.8) and the obvious extension of (4.2) in the same way that (3.16a) follows from (3.15) and (3.13).

The next result is an easy consequence of (4.9) and (4.10). See Azema [2] for the original result of this type. Related results can be found in [1 and 14].

(4.12) THEOREM. Let $Z \in \mathcal{K}^{m}$. Then ${ }^{\circ} \hat{p} Z 1_{\Lambda}$ and ${ }^{\hat{p} \circ} Z 1_{\Lambda}$ are $Q_{m}$-indistinguishable.

ProOF. We may assume that $Z \in\left(\mathcal{M}^{m}\right)^{+}$. In fact, since $\mathcal{M}^{m}=\mathcal{M}^{0} \vee I^{m}$ and since both the optional and the copredictable projection preserve $I^{m}$, we may even assume that $Z \in\left(M^{0}\right)^{+}$. Using the explicit formulae (4.9) and (4.11) a straightforward calculation reveals that a common version of ${ }^{\circ} \hat{p} Z 1_{\Lambda}$ and ${ }^{\hat{p} \circ} Z 1_{\Lambda}$ is given by

$$
(t, w) \rightarrow 1_{\Lambda}(t, w) \int_{\Omega} \int_{\hat{\Omega}} Z_{t}\left(\sigma_{-t} w^{\prime \prime} / t / \sigma_{-t} w^{\prime}\right) \hat{P}^{\bar{Y}_{t}(w)}\left(d w^{\prime \prime}\right) P^{\bar{Y}_{t}(w)}\left(d w^{\prime}\right)
$$

Our second application of the dual family $\left(\hat{P}^{x}\right)$ concerns the regularity of RadonNikodym derivatives $d \xi / d m$ when $\xi \in$ Exc. 
(4.13) LEMMA. Let $\xi \in \operatorname{Exc}$ with $\xi<<m$. There exists a version $\psi \in \mathcal{E}^{+}$of $d \xi / d m$ such that for each $x \in E, t \rightarrow \psi\left(\hat{X}_{t}\right)$ has left limits on $] 0,+\infty[$ and right limits on $\left[0,+\infty\left[\right.\right.$, a.s. $\hat{P}^{x}$.

ProOF. Let $\psi_{0}$ be any $\mathcal{E}$-measurable version of $d \xi / d m$. Let $g \in \mathcal{E}^{+}$. Since $\left(P_{t}\right)$ and $\left(\hat{P}_{t}\right)$ are duals relative to $m$ and since $\xi \in$ Exc, we have

$$
m\left(\psi_{0} g\right)=\xi(g) \geq \xi\left(P_{t} g\right)=m\left(\psi_{0} P_{t} g\right)=m\left(\hat{P}_{t} \psi_{0} \cdot g\right)
$$

and

$$
m\left(g \hat{P}_{t} \psi_{0}\right)=\xi\left(P_{t} g\right) \uparrow \xi(g)=m\left(\psi_{0} \cdot g\right) \quad \text { as } t \downarrow 0 \text {. }
$$

It follows that $\psi_{0} \geq \hat{P}_{t} \psi_{0}$ a.e. $m$, and that $\hat{P}_{t} \psi_{0} \uparrow \psi_{0}$ a.e. $m$ as $t \downarrow 0$ through any fixed sequence. By (6.18) and (6.19) of [29] there exists $\psi \in \mathcal{E}^{+}$such that $\psi$ is excessive for $\left(\hat{P}_{t}\right)$ and such that $\psi=\psi_{0}$ a.e. $m$. Thus $\psi$ is a version of $d \xi / d m$. Moreover, the arguments of Chung and Glover in Lemmas 1 and 2 of [9] show that $t \rightarrow \psi\left(\hat{X}_{t}\right)$ has the required left and right limits a.s. $\hat{P}^{x}$ for each $x \in E$. (Although a left continuous moderate Markov process is assumed in [9], the arguments there use only the predictability of $\psi\left(\hat{X}_{t}\right)$.)

A further refinement of (4.13) requires the following lemma.

(4.14) LEMMA. Let $B_{0}$ be a Borel m-polar set. Then there exists a Borel $m$ polar set $B \supset B_{0}$ such that $E \backslash B$ is absorbing for $X$ and for $\hat{X}$; that is, for all $x \in E \backslash B$,

$$
\begin{aligned}
& P^{x}\left(X_{t} \in B, \text { some } t \geq 0\right)=0, \\
& \hat{P}^{x}\left(\hat{X}_{t} \in B, \text { some } t>0\right)=0 .
\end{aligned}
$$

ProOF. By $[\mathbf{3 2}, \S 2,(1),(13)]$, if $A$ is $m$-polar then there is a Borel $m$-polar set $A^{\prime} \supset A$ such that $E \backslash A^{\prime}$ is absorbing for $\hat{X}$. Similarly, by [29, (6.12)], if $A$ is Borel $m$-polar then there is a Borel $m$-polar set $A^{\prime \prime} \supset A$ with $E \backslash A^{\prime \prime}$ absorbing for $X$. Given a Borel $m$-polar set $B_{0}$ define inductively

$$
\begin{array}{ll}
B_{2 n+1}=\left(B_{2 n}\right)^{\prime}, & n \geq 0 \\
B_{2 n}=\left(B_{2 n-1}\right)^{\prime \prime}, & n \geq 1 ;
\end{array}
$$

and finally $B=\bigcup_{n=1}^{\infty} B_{n}$. It is a simple matter to check that $B$ has the desired properties.

(4.15) PROPOSITION. Let $\xi \in$ Exc with $\xi<<m$. Then there exists a version $\phi \in \mathcal{E}^{+}$of $d \xi / d m$ such that

(i) $t \rightarrow \phi\left(\bar{Y}_{t}\right)$ has left and right limits on $\mathbf{R}$, and is rc on $\Lambda$, a.e. $Q_{m}$;

(ii) there is a Borel $m$-polar set $B$, with $E \backslash B$ absorbing for $X$ and for $\hat{X}$, such that for $x \in E \backslash B$

(a) $\phi(x)<\infty$;

(b) $t \rightarrow \phi\left(\hat{X}_{t}\right)$ has left and right limits on $\left[0,+\infty\left[\right.\right.$ and is lc on $\hat{\Lambda}$, a.s. $\hat{P}^{x}$.

PROOF. Let $\psi$ be chosen as per (4.13). It is easy to check that $t \rightarrow \psi\left(\bar{Y}_{t}\right)$ has left and right limits on ] $-\infty, \beta\left[\right.$ a.e. $Q_{m}$. We may thus define a process $\left(H_{t}\right)_{t \in \mathbf{R}}$ by

$$
H_{t}=\liminf _{s \downarrow t} \psi\left(\bar{Y}_{s}\right)
$$


and note that $\left(H_{t}\right)$ is rc a.e. $Q_{m}, O^{m} \cap \hat{P}^{m}$-measurable, and homogeneous. By (3.21) there is a function $\phi \in \mathcal{E}^{+}$such that $H 1_{\Lambda}$ is $Q_{m}$-indistinguishable from $\left(\phi\left(\bar{Y}_{t}\right)\right)$. Since $t \rightarrow \psi\left(\bar{Y}_{t}\right)$ has left and right limits on $]-\infty, \beta\left[\right.$, the set $\left\{t: \psi(\bar{Y})_{t} \neq \psi(\bar{Y})_{t-}\right\}$ is at most countable (for each $w$ ). This fact, combined with the homogeneity of $\psi\left(\bar{Y}_{t}\right)$ and the stationarity of $Q_{m}$, shows that

$$
0=Q_{m}\left(\psi\left(\bar{Y}_{t}\right) \neq H_{t}\right)=Q_{m}\left(\psi\left(\bar{Y}_{t}\right) \neq \phi\left(\bar{Y}_{t}\right)\right)
$$

for each $t \in \mathbf{R}$. Thus $\phi$ is a version of $d \xi / d m$ which satisfies (i). Clearly $\{\phi=+\infty\}$ is a Borel $m$-polar set, as is $\left\{x: \hat{P}^{x}\left(G^{c}\right)>0\right\}$, where

$$
G=\left\{w \in \hat{\Omega}: t \rightarrow \phi\left(\hat{X}_{t}(w)\right) \text { has left and right limits on }[0,+\infty[\text { and is lc on } \hat{\Lambda}\} \text {. }\right.
$$

(See the proof of (A.6) in the appendix.) By (4.18) we may enclose the $m$-polar set $\{\phi=+\infty\} \cup\left\{x: \hat{P}^{x}\left(G^{c}\right)>0\right\}$ in a Borel $m$-polar set $B$ which has the properties described in (ii).

(4.16) REMARKS. (a) Dynkin and Getoor [17] assume the existence of a version of $d \xi / d m$ with the properties listed in (4.15). Thus (4.15) removes the need for hypotheses $1.7 \mathrm{~A}$ of [17], at least when the basic process $X$ is a Borel right process.

(b) With $\phi$ as in (4.15) it follows that $\phi$ is finely continuous at each point of a set whose complement is $m$-polar. In particular for any $t \geq 0$ the set $\{\phi>t\}$ differs from its fine interior by an $m$-polar set.

5. Random measures; Palm measures. In this section we develop some of the theory of homogeneous random measures over the stationary process $\left(Y, Q_{m}\right)$. When $X$ is in weak duality (relative to $m$ ) with a second Borel right process, such random measures have been considered in $[\mathbf{1}, \mathbf{2 9}, \mathbf{3 8}, \mathbf{3 2}]$. Under hypotheses close to ours, Dynkin and Getoor [17] have considered diffuse random measures. For related matters see $[\mathbf{2}, \mathbf{5}, \mathbf{1 4}, \mathbf{1 1}, \mathbf{2 1}, \mathbf{2 2}, \mathbf{3 5}]$.

In previous treatments only random measures carried by $\rrbracket \alpha, \beta \llbracket$ have been studied systematically. For such homogeneous random measures the Revuz measure suffices to characterize the class of random measures under study. For the applications we have in mind it is necessary to allow random measures that charge $\llbracket \alpha \rrbracket$. For this reason we take the Palm measure as a basic tool in our study. Palm measures are familiar in the theory of flows $[\mathbf{2 1}, \mathbf{2 2}, \mathbf{3 5}]$ and have been used in our context in $[1]$.

Throughout this section $m \in$ Exc is fixed and all considerations are relative to $\left(Y, Q_{m}\right)$. We begin with the basic

(5.1) Definition. A random measure (RM) is a positive kernel $\kappa=\kappa(w, B)$ from $\left(W, g^{m}\right)$ to $(\mathbf{R}, B)$ such that

(i) the measure $\kappa(w, \cdot)$ is carried by $[\alpha(w), \beta(w)[\cap \mathbf{R}$ for each $w \in W$;

(ii) there is a countable collection of kernels $\left\{\kappa^{n}: n \geq 1\right\}$ from $\left(W, \mathcal{G}^{m}\right)$ to $(\mathbf{R}, B)$ with $Q_{m}\left(\kappa^{n}(\mathbf{R})=\infty\right)=0, \forall n$, such that $\kappa=\sum_{n=1}^{\infty} \kappa^{n}$.

Two random measures $\kappa$ and $\gamma$ are $Q_{m}$-indistinguishable if $\kappa(w, \cdot)=\gamma(w, \cdot)$ for $Q_{m}$-a.e. $w$. Note that condition (ii) above implies

(ii) ${ }^{\prime}$ there is a countable collection $\left\{\kappa^{n}\right\}$ of kernels from $\left(W, \mathcal{G}^{m}\right)$ to $(\mathbf{R}, B)$ with $\kappa^{n}(w, \mathbf{R}) \leq 1, \forall w, \forall n$, such that $\sum_{n=1}^{\infty} \kappa^{n}$ and $\kappa$ are $Q_{m}$-indistinguishable.

Indeed, if (ii) holds we set $a_{n k}(w)=\left(\kappa^{n}(w, \mathbf{R}) \wedge k\right)-\left(\kappa^{n}(w, \mathbf{R}) \wedge(k-1)\right)$ so that $a_{n k}(w) \leq 1$ and $\sum_{k=1}^{\infty} a_{n k}(w)=\kappa^{n}(w, \mathbf{R})$. Now set

$$
\kappa_{n k}(w, \cdot)=\left(a_{n k}(w) / \kappa^{n}(w, \mathbf{R})\right) \kappa^{n}(w, \cdot)
$$


$\left(=0\right.$ if $\left.\kappa^{n}(w, \mathbf{R})=+\infty\right)$ and note that $\kappa_{n k}(w, \mathbf{R}) \leq 1$ and $\sum_{n, k} \kappa_{n k}(w, \cdot)=\kappa(w, \cdot)$ if $\kappa^{n}(w, \mathbf{R})<\infty$ for all $n$. Thus, $\left\{\kappa_{n k}: n \geq 1, k \geq 1\right\}$ is a collection satisfying (ii)'.

The countable finiteness conditions (ii) and (ii)' justify later uses of Fubini's theorem.

Associated with a RM $\kappa$ is its Doleans measure $M_{\kappa}$ defined by

$$
M_{\kappa}(Z)=Q_{m}\left(\int_{\mathbf{R}} Z_{t} \kappa(d t)\right), \quad Z \in\left(\mathcal{M}^{m}\right)^{+} .
$$

We sometimes write $\langle Z, \kappa\rangle$ for $M_{\kappa}(Z)$. Note that $M_{\kappa}$ vanishes on $I^{m}$ and that $M_{\kappa}$ is carried by $\Gamma \in \mathcal{M}^{m}$ if and only if $\kappa$ is so carried, a.s. $Q_{m}$. In particular, $M_{\kappa}$ is carried by $\llbracket \alpha, \beta \llbracket$.

(5.2) DEFInItion. A RM $\kappa$ is $\sigma$-integrable over a class $S \subset\left(\mathcal{M}^{m}\right)^{+}$provided there is a strictly positive $Z \in S$ such that $M_{\kappa}(Z)<\infty$. Actually $Z>0$ need hold only on a set carrying $\kappa$. The class of RM's $\sigma$-integrable over $S$ is denoted $\sigma I(S)$. Clearly $\sigma I\left(\hat{p}^{m}\right)=\sigma I\left(\hat{p}^{0}\right), \sigma I\left(O^{m}\right)=\sigma I\left(O^{0}\right)$ because of $(3.1)$.

The following result collects some facts about Doleans measures which may be proved by standard methods. See [12, Chapter VI] or [38].

(5.3) Proposition. (a) Let $\kappa^{1}$ and $\kappa^{2}$ be $R M^{\prime}$ 's in $\sigma I\left(\mathcal{M}^{m}\right)$. Suppose that $M_{\kappa^{1}}=M_{\kappa^{2}}$. Then $\kappa^{1}$ and $\kappa^{2}$ are $Q_{m}$-indistinguishable.

(b) Let $M$ be a $\sigma$-finite measure on $\mathcal{M}^{m}$ which is carried by $\llbracket \alpha, \beta \llbracket$ and which charges no $Q_{m}$-evanescent set. Then $M$ is the Doleans measure of a unique $R M$ $\kappa \in \sigma I\left(\mathcal{M}^{m}\right)$.

Suppose that $\kappa \in \sigma I\left(\hat{p}^{m}\right)$. We say that $\kappa$ is $\left(Q_{m}\right)$-copredictable if $\langle Z, \kappa\rangle=$ $\left\langle{ }^{\hat{p}} Z, \kappa\right\rangle$ for all $Z \in\left(\mathcal{M}^{m}\right)^{+}$. More generally, consider the measure $M$ defined by

$$
M(Z)=\left\langle{ }^{\hat{p}} Z, \kappa\right\rangle, \quad Z \in\left(\mathcal{M}^{m}\right)^{+} .
$$

Since ${ }^{\hat{p}} Z \in I^{m}$ if $Z \in I^{m}$, it is clear that $M$ satisfies the hypotheses of $(5.3 \mathrm{~b})$. Thus there is a unique $\mathrm{RM}, \kappa^{\hat{p}}$, whose Doleans measure is $M$; evidently $\kappa^{\hat{p}}$ is copredictable. We refer to $\kappa^{\hat{p}}$ as the $Q_{m}$-dual copredictable projection of $\kappa$.

The "big shift" operates on random measures by

$$
\left(\Sigma_{s} \kappa\right)(w, B)=\kappa\left(\sigma_{s} w, B-s\right) .
$$

It is clear that if $\kappa$ is a $\mathrm{RM}$ then so is $\Sigma_{s} \kappa$ for any $s \in \mathbf{R}$. Moreover,

$$
\left\langle Z, \Sigma_{s} \kappa\right\rangle=\left\langle\Sigma_{-s} Z, \kappa\right\rangle .
$$

It follows from (5.4) that if $S \subset \mathcal{M}^{m}$ is invariant under $\Sigma_{s}$ for all $s \in \mathbf{R}$, then $\sigma I(S)$ is also invariant under $\Sigma_{s}$ for all $s \in \mathbf{R}$. We say that a RM, $\kappa$, is homogeneous (and abbreviate HRM) if $\Sigma_{s} \kappa=\kappa$ (modulo $I^{m}$ ) for each $s \in \mathbf{R}$. The following summary is an immediate consequence of the definitions and (5.3).

(5.5) Proposition. (a) If $\kappa \in \sigma I\left(\hat{p}^{m}\right)$, then $\Sigma_{s} \kappa \in \sigma I\left(\hat{p}^{m}\right)$, and $\left(\Sigma_{s} \kappa\right)^{\hat{p}}=$ $\Sigma_{s}\left(\kappa^{\hat{p}}\right)$, modulo $I^{m}$, for each $s \in \mathbf{R}$.

(b) $A R M \kappa \in \sigma I\left(\mathcal{M}^{m}\right)$ is homogeneous if and only if $\Sigma_{s} M_{\kappa}=M_{\kappa}$ for all $s \in \mathbf{R}$.

The dual notions involving optional random measures are more delicate because our optional projection is defined only on $\Lambda$. The following definition may be regarded as provisional but it is adequate for our purposes. 
(5.6) Definition. Let $\kappa$ be a RM. Then $\kappa$ is optional provided

(i) $\left(1_{\Lambda^{c}} * \kappa\right)\{\alpha\}$ is $\mathcal{G}_{\alpha}^{m}$-measurable and finite a.e. $Q_{m}$;

(ii) $1_{\Lambda} * \kappa \in \sigma I\left(O^{m}\right)$ and $\left\langle Z, 1_{\Lambda} * \kappa\right\rangle=\left\langle{ }^{\circ} Z, 1_{\Lambda} * \kappa\right\rangle$ for all $Z \in\left(\mathcal{M}^{m}\right)^{+}$.

Here and subsequently, if $Z \in\left(\mathcal{M}^{m}\right)^{+}$, then $Z * \kappa$ denotes the RM $Z_{t} \kappa(d t)$.

Let $\kappa$ be a $\mathrm{RM}$ in $\sigma I\left(\mathrm{O}^{m}\right)$ which is carried by $\Lambda$. The dual optional projection of $\kappa$, denoted by $\kappa^{\circ}$, is uniquely determined by

$$
\left\langle Z, \kappa^{\circ}\right\rangle=\left\langle{ }^{\circ} Z, \kappa\right\rangle, \quad Z \in\left(\mathcal{M}^{m}\right)^{+} .
$$

See (5.3) and recall that ${ }^{\circ} Z \in I^{m}$ if $Z \in I^{m}$. Clearly $\kappa^{\circ}$ is carried by $\Lambda$ and is an optional RM. Since $\Lambda$ is perfectly homogeneous, the mapping $\kappa \rightarrow \kappa^{\circ}$ commutes with each $\Sigma_{s}$. We leave it to the reader to write down the optional version of (5.5); in particular if $\kappa$ is a HRM and if $\kappa^{\circ}$ exists, then $\kappa^{\circ}$ is a HRM.

Here is the "dual" to (4.12).

(5.7) Proposition. Let $\kappa$ be a $R M$ in $\sigma I\left(O^{m}\right) \cap \sigma I\left(\hat{p}^{m}\right)$ which is carried by $\Lambda$. Then $\left(\kappa^{\hat{p}}\right)^{\circ}=\left(\kappa^{\circ}\right)^{\hat{p}}$ up to $Q_{m}$-indistinguishability.

PROOF. This follows immediately from (4.12) since each of $\kappa, \kappa^{\circ}, \kappa^{\hat{p}}, \kappa^{\circ \hat{p}}$, and $\kappa^{\hat{p} \circ}$ is carried by $\Lambda \in \mathcal{O}^{0} \cap \hat{p}^{0}$.

A second, and more convenient, "characteristic" of a HRM $\kappa$ is its Palm measure introduced in the next

(5.8) Definition. Let $\kappa$ be an optional HRM. The Palm measure of $\kappa, \mathbf{P}_{\kappa}$, is the measure on $\mathcal{G}^{0}$ defined by

$$
\mathbf{P}_{\kappa}(F)=Q_{m} \int_{[0,1]} F \circ \tau_{t} \kappa(d t), \quad F \in\left(\mathcal{G}^{0}\right)^{+} .
$$

Note that $\mathbf{P}_{\kappa}$ is carried by $\{\alpha=0\}$ so that $b_{0}\left(\mathbf{P}_{\kappa}\right)=\mathbf{P}_{\kappa}$. The measure $\mathbf{P}_{\kappa}$ is similar to measures considered in $[\mathbf{2 1}, \mathbf{2 2}, \mathbf{3 5}, \mathbf{1}]$ but is "one-sided" in that $\tau_{t}$ is used in (5.9) rather than $\sigma_{t}$. The appropriate classes of "exceptional sets" for the class of Palm measures are defined next (cf. [21]).

(5.10) DEFINITION. (a) A set $A \in \mathcal{G}^{0}$ is $m$ - $\tau$-polar provided the process $\left(1_{A} \circ \tau_{t} 1_{\rrbracket \alpha, \beta \llbracket}(t)\right)_{t \in \mathbf{R}}$ is $Q_{m}$-evanescent.

(b) A set $A \in \mathcal{G}^{0}$ is $m$ - $\tau$-evanescent provided the process $\left(1_{A} \circ \tau_{t} 1_{\llbracket \alpha, \beta \llbracket}(t)\right)_{t \in \mathbf{R}}$ is $Q_{m}$-evanescent.

Clearly, if $\kappa$ is an optional HRM then $\mathbf{P}_{\kappa}(A)=0$ whenever $A$ is $m$ - $\tau$-evanescent. It is easy to check that $A$ is $m$ - $\tau$-evanescent if and only if $A$ is $m$ - $\tau$-polar and $Q_{\nu}(A)=0$, where $\nu=\left(\nu_{t}\right)$ is the entrance law such that $m_{p}=\int_{0}^{\infty} \nu_{t} d t\left(m_{p}\right.$ is the purely excessive part of $m$ ).

(5.11) Proposition. Let $\kappa$ be an optional HRM with Palm measure $\mathbf{P}_{\kappa}$. Then

(a) for all $f \in\left(B \otimes \mathcal{G}^{0}\right)^{+}$,

$$
Q_{m}\left(\int_{\mathbf{R}} f\left(t, \tau_{t}\right) \kappa(d t)\right)=\int_{\mathbf{R}} d t \int_{W} \mathbf{P}_{\kappa}(d w) f(t, w) .
$$

(b) $\kappa \in \sigma I\left(\hat{p}^{m}\right)$ if and only if $\mathbf{P}_{\kappa}$ is $\sigma$-finite. In this case $\mathbf{P}_{\kappa}=\mathbf{P}_{\kappa^{\hat{p}}}$.

(c) Let $\kappa \in \sigma I\left(\hat{P}^{m}\right)$. Then $\kappa$ is carried by $\Lambda$ if and only if $\mathbf{P}_{\kappa}$ charges no $m-\tau$ polar set. 
ProOF. (a) Let $\mu$ denote the measure on $B \otimes \mathcal{G}^{0}$ defined by the left side of (5.12), as a function of $f$. Let $\left\{A_{n}: n \in \mathbf{N}\right\}$ be a partition of $W$ into $\mathcal{G}^{m}$-measurable sets of finite $Q_{m}$ measure. Recall that $\kappa=\sum_{i} \kappa^{i}$ where each $\kappa^{i}$ is a kernel with $\kappa^{i}(w, \mathbf{R}) \leq 1$ for all $w$. Set

$$
\mu_{n}^{i}(f)=Q_{m}\left(\int_{\mathbf{R}} f\left(t, \tau_{t}\right) \kappa^{i}(d t) ; A_{n}\right)
$$

so that $\mu_{n}^{i}(\mathbf{R} \times W)<\infty$ and $\sum_{i, n} \mu_{n}^{i}=\mu$. That is, $\mu$ is a countable sum of finite measures. Owing to the homogeneity of $\kappa$ and the $\left(\sigma_{s}\right)$-invariance of $Q_{m}, \mu$ is translation invariant in its first coordinate (i.e., if $f_{s}(t, w)=f(t+s, w)$, then $\mu\left(f_{s}\right)=\mu(f)$ for all $\left.s \in \mathbf{R}\right)$. Thus the measure on $\mathbf{R}$ defined by $\varphi \rightarrow \mu(\varphi \otimes F)$ $((\varphi \otimes F)(t, w)=\varphi(t) F(w))$ is a multiple of Lebesgue measure (cf. [25]). Clearly this multiple must be $\mu\left(1_{0,1]} \otimes F\right)=\mathbf{P}_{\kappa}(F)$. Thus (5.12) is valid if $f$ has the form $f(t, w)=\varphi(t) F(w)$. The validity of (5.12) for all $f \in\left(B \otimes \mathcal{G}^{0}\right)^{+}$now follows by a result of Getoor [25].

(b) Suppose first that $\mathbf{P}_{\kappa}$ is $\sigma$-finite. Choose $H>0$ with $H \in \mathcal{G}^{0}, \mathbf{P}_{\kappa}(H)<\infty$. Let $g>0$ be Lebesgue integrable over $\mathbf{R}$ and define $Z_{t}=g(t) H \circ \tau_{t}, t \in \mathbf{R}$. Evidently $Z \in \hat{p}^{0}$ and

$$
\begin{aligned}
M_{\kappa}(Z) & =Q_{m}\left(\int_{\mathbf{R}} g(t) H \circ \tau_{t} \kappa(d t)\right) \\
& =\lambda(g) \mathbf{P}_{\kappa}(H)<\infty,
\end{aligned}
$$

because of (5.12). Here $\lambda$ denotes Lebesgue measure on $\mathbf{R}$. Conversely, suppose that $\kappa \in \sigma I\left(\hat{p}^{m}\right)$. Then there is a process $Z \in \hat{p}^{0}$ with $Z>0$ and $M_{\kappa}(Z)<\infty$. Using (5.12) and the fact that $Z_{t}=Z_{t} \circ b_{t}$ since $Z \in \hat{\rho}^{0}$, we may compute

$$
\begin{aligned}
\infty & >M_{\kappa}(Z)=Q_{m}\left(\int Z_{t} \circ b_{t} \kappa(d t)\right) \\
& =Q_{m}\left(\int Z_{t} \circ \sigma_{-t} \circ \tau_{t} \kappa(d t)\right)=\int_{\mathbf{R}} d t \mathbf{P}_{\kappa}\left(Z_{t} \circ \sigma_{-t}\right) .
\end{aligned}
$$

Thus $\mathbf{P}_{\kappa}\left(Z_{t} \circ \sigma_{-t}\right)<\infty$ for Lebesgue a.e. $t \in \mathbf{R}$. Since $Z>0, \mathbf{P}_{\kappa}$ is $\sigma$-finite. Now if $\kappa \in \sigma I\left(\hat{p}^{m}\right)$ then $\kappa^{\hat{p}}$ exists and $\left\langle Z, \kappa^{\hat{p}}\right\rangle=\left\langle\hat{p}^{\hat{p}} Z, \kappa\right\rangle$. But $t \rightarrow F \circ \tau_{t}$ is copredictable if $F \in\left(\mathcal{G}^{0}\right)$ and so

$$
\begin{aligned}
\mathbf{P}_{\kappa \hat{p}}(F) & =Q_{m}\left(\int_{[0,1]} F \circ \tau_{t} \kappa^{\hat{p}}(d t)\right) \\
& =Q_{m}\left(\int_{[0,1]} F \circ \tau_{t} \kappa(d t)\right)=\mathbf{P}_{\kappa}(F) .
\end{aligned}
$$

(c) In view of (b) and the copredictability of $\Lambda$, we may assume without loss of generality that $\kappa$ is copredictable. Suppose first that $\kappa$ is carried by $\Lambda$. Let $A \in \mathcal{G}^{0}$ 
be $m$ - $\tau$-polar. Then

$$
\begin{aligned}
\mathbf{P}_{\kappa}\left(1_{A} l_{0} 1_{\left\{l_{0}>0\right\}}\right) & =Q_{m}\left(\int_{] 0,1]} 1_{A} \circ \tau_{t} l_{t} 1_{\Lambda}(t) \kappa(d t)\right) \\
& =Q_{m}\left(\int_{] 0,1]} 1_{A} \circ \tau_{t} l_{t} \kappa(d t)\right) \\
& =Q_{m}\left(\int_{j 0,1]} 1_{A} \circ \tau_{t} 1_{\rrbracket \alpha, \beta \llbracket}(t) \kappa(d t)\right) \\
& =0,
\end{aligned}
$$

and

$$
\begin{aligned}
\mathbf{P}_{\kappa}\left(A ; l_{0}=0\right) & =Q_{m}\left(\int_{] 0,1]} 1_{A} \circ \tau_{t} 1_{\llbracket \alpha, \beta \llbracket \backslash \Lambda}(t) \kappa(d t)\right) \\
& =0 .
\end{aligned}
$$

It follows that $\mathbf{P}_{\kappa}(A)=0$. Conversely, since $\tau_{t}^{-1}\left\{l_{0}=0\right\}=\left\{l_{t}=0\right\}$ and since $\Lambda=\left\{(t, w): l_{t}(w)>0\right\} \supset \rrbracket \alpha, \beta \llbracket$, the set $\left\{l_{0}=0\right\}$ is $m$ - $\tau$-polar. Thus, if $\mathbf{P}_{\kappa}$ charges no $m$ - $\tau$-polar set, then

$$
\begin{aligned}
Q_{m} \int_{\mathbf{R}} g(t) H \circ \tau_{t} \kappa(d t) & =\lambda(g) \mathbf{P}_{\kappa}(H) \\
& =\lambda(g) \mathbf{P}_{\kappa}\left(H: l_{0}>0\right) \\
& =Q_{m} \int_{\mathbf{R}} g(t) 1_{\Lambda}(t) H \circ \tau_{t} \kappa(d t),
\end{aligned}
$$

where $g \in B^{+}$and $H \in\left(\mathcal{G}^{0}\right)^{+}$. It follows that $\kappa$ is carried by $\Lambda$.

Although not labelled as such, a special type of Palm measure was considered in [19]. Let $T$ be a $Q_{m}$-optional time which is intrinsic in the sense that $\alpha \leq T<\beta$ if $T<+\infty$ and $t+T \circ \sigma_{t}=T$ for all $t \in \mathbf{R}$. For example $\inf \left(t \geq \alpha: \bar{Y}_{t} \in B\right)$ is such a time if $B \in \mathcal{E}^{+}$. Then $\kappa=1_{\{T \in \mathbf{R}\}} \varepsilon_{T}$ is an optional HRM with Palm measure

$$
\Pi^{T}(F)=Q_{m}\left(F \circ \tau_{T} ; 0<T \leq 1\right) .
$$

It is shown in Theorem $(2.4)$ of $[\mathbf{1 9}]$ that $\Pi^{T}$ is a $\sigma$-finite measure carried by $\{\alpha=0\}$ and that, under $\Pi^{T},\left(Y_{t}\right)_{t>0}$ is Markovian with semigroup $\left(P_{t}\right)$. As we shall see, the Markovian nature of $\left(Y_{t}\right)_{t>0}$ is present for the Palm measure $\mathbf{P}_{\kappa}$ of a general optional HRM.

Let $\kappa$ be an optional HRM with Palm measure $\mathbf{P}_{\kappa}$. Define a family of measures on $E$ by

$$
\mu_{t}^{\kappa}(f)=\mathbf{P}_{\kappa}\left(f \circ Y_{t}\right), \quad t>0, f \in \mathcal{E}^{+},
$$

with $\mu_{t}^{\kappa}=0$ if $t \leq 0$.

(5.13) Proposition. Suppose that each $\mu_{t}^{\kappa}$ is $\sigma$-finite. Then $\mu^{\kappa}=\left(\mu_{t}^{\kappa}\right)$ is an entrance law, $\mathbf{P}_{\kappa}$ is $\sigma$-finite, and $\mathbf{P}_{\kappa}=Q_{\mu^{\kappa}}$ (the Kuznetsov measure of $\mu^{\kappa}$ ).

Proof. Note that $\mathbf{P}_{\kappa}$, being carried by $\{\alpha=0\}$, does not charge $\{[\Delta]\}$. We now claim that for each $f \in \mathcal{E}^{+}, F \in\left(\mathcal{G}^{0}\right)^{+}$, and $s>0$,

$$
\mathbf{P}_{\kappa}\left(f \circ Y_{s} F \circ \theta_{s}\right)=\mathbf{P}_{\kappa}\left(f \circ Y_{s} P^{Y_{s}}(F)\right) \text {. }
$$


To see this decompose $\kappa$ as $\kappa^{1}+\kappa^{2}=1_{\Lambda^{c}} * \kappa+1_{\Lambda} * \kappa$. Let $\mathbf{P}^{1}+P^{2}$ be the corresponding decomposition of $\mathbf{P}_{\kappa}$ so that $\mathbf{P}^{1}=1_{\left\{l_{0}=0\right\}} \mathbf{P}_{\kappa}, \mathbf{P}^{2}=1_{\left\{l_{0}>0\right\}} \mathbf{P}_{\kappa}$. Using the strong Markov property of $Q_{m}$ at time $\alpha+s$ we compute

$$
\begin{aligned}
\mathbf{P}^{1}\left(f \circ Y_{s} F \circ \theta_{s}\right) & =Q_{m}\left(\int_{\mathrm{j} 0,1]} f \circ Y_{t+s} F \circ \tau_{t+s} \kappa^{1}(d t)\right) \\
& =Q_{m}\left(f \circ Y_{\alpha+s} F \circ \tau_{\alpha+s} \kappa^{1}\{\alpha\} ; 0<\alpha \leq 1\right) \\
& =Q_{m}\left(f \circ Y_{\alpha+s} P^{Y_{\alpha+s}}(F) \kappa^{1}\{\alpha\} ; 0<\alpha \leq 1\right) \\
& =Q_{m}\left(\int_{0,1]} f \circ Y_{t+s} P^{Y_{t+s}}(F) \kappa^{1}(d t)\right) \\
& =\mathbf{P}^{1}\left(f \circ Y_{s} P^{Y_{s}}(F)\right) .
\end{aligned}
$$

As for $\mathbf{P}^{2}$, note that the processes

$$
Z_{t}=1_{\Lambda}(t) f \circ Y_{t+s} F \circ \tau_{t+s}, \quad Z_{t}^{1}=1_{\Lambda}(t) f \circ Y_{t+s} P^{Y_{t+s}}(F)
$$

have the same optional projection, namely $1_{\Lambda}(t) P^{\bar{Y}_{t}}\left(f \circ Y_{s} P^{Y_{s}}(F)\right)$. Thus, since $1_{\Lambda} * \kappa$ is optional,

$$
\begin{aligned}
\mathbf{P}^{2}\left(f \circ Y_{s} F \circ \theta_{s}\right) & =Q_{m} \int_{[0,1]} Z_{t} \kappa^{2}(d t) \\
& =Q_{m} \int_{[0,1]}{ }^{\circ} Z_{t} \kappa^{2}(d t) \\
& =Q_{m} \int_{j 0,1]}^{\circ}\left(Z^{1}\right)_{t} \kappa^{2}(d t) \\
& =Q_{m} \int_{] 0,1]} Z_{t}^{1} \kappa^{2}(d t)=\mathbf{P}^{2}\left(f \circ Y_{s} P^{Y_{s}}(F)\right) .
\end{aligned}
$$

Adding the above results for $\mathbf{P}^{1}$ and $\mathbf{P}^{2}$ we obtain (5.14). Taking $F=G \circ Y_{u}(u>0)$ and $f=1_{E}$ in (5.14) we see that $\left(\mu_{t}^{\kappa}\right)$ is an entrance law. This fact and an easy induction using (5.14) show that $\mathbf{P}_{\kappa}$ and $Q_{\mu^{\kappa}}$ have the same finite dimensional distributions. We may invoke the uniqueness in (2.1) to conclude that $\mathbf{P}_{\kappa}=Q_{\mu^{\kappa}}$.

Our interest lies mainly in the class of optional HRM's whose Palm measures satisfy the condition

$$
\xi \equiv \int_{0}^{\infty} \mu_{t}^{\kappa} d t \quad \text { is } \sigma \text {-finite. }
$$

The measure $\xi$ is then purely excessive and each $\mu_{t}^{\kappa}$ is $\sigma$-finite (choose $q>0$ with $\xi(q)<\infty$; then $U q>0$ and $\left.\mu_{t}^{\kappa} U q=\xi P_{t}(q) \leq \xi(q)<\infty\right)$. Here is our fundamental result characterizing this class.

(5.15) THEOREM. Let $\xi$ be a purely excessive measure so that $\xi=\int_{0}^{\infty} \mu_{t} d t$ where $\mu=\left(\mu_{t}\right)$ is an entrance law. Let $Q_{\mu}$ denote the corresponding Kuznetsov measure. Then $Q_{\mu}$ is the Palm measure of an optional HRM $\kappa$ if and only if $Q_{\mu}$ charges no $m-\tau$-evanescent set. In this case $\kappa \in \sigma I\left(\hat{\mathcal{P}}^{m}\right)$ and $\kappa$ may be chosen to be copredictable. With this choice $\kappa$ is uniquely determined. 
PROOF. The "only if" part of the assertion follows from the preceding discussion. Conversely, suppose that $Q_{\mu}$ charges no $m$ - $\tau$-evanescent set. Our plan is to decompose $Q_{\mu}$ as

$$
Q^{1}+Q^{2}=1_{\left\{l_{0}=0\right\}} Q_{\mu}+1_{\left\{l_{0}>0\right\}} Q_{\mu}
$$

and then to produce HRM's $\kappa^{1}$ and $\kappa^{2}$ with Palm measures $Q^{1}$ and $Q^{2}$ respectively. Recall that $m_{p}$, the purely excessive part of $m$, can be written as $\int_{0}^{\infty} \nu_{t} d t$ where $\nu=\left(\nu_{t}\right)$ is an entrance law with Kuznetsov measure $Q_{\nu}$.

(a) First consider $Q^{1}$. We claim that $Q^{1}<<Q_{\nu}$. For if $A \in \mathcal{G}^{0}$ with $Q_{\nu}(A)=0$, then $Q_{m}\left(\tau_{\alpha}^{-1} A ; \alpha \in \mathbf{R}\right)=0$ by (2.5). By a previous remark, $\left\{l_{0}=0\right\}$ is $m$ - $\tau$-polar. Thus $A \cap\left\{l_{0}=0\right\}$ is $m$ - $\tau$-evanescent and so $0=Q_{\mu}\left(A \cap\left\{l_{0}=0\right\}\right)=Q^{1}(A)$. That is, $Q^{1}<<Q_{\nu}$. Let $J \in\left(\mathcal{G}^{0}\right)^{+}$denote a version of $d Q^{1} / d Q_{\nu}$. Note that both $Q^{1}$ and $Q_{\nu}$ are $\sigma$-finite on $\mathcal{G}_{t+}^{0} \cap\{t<\beta\}$ for each $t>0$. Fix $t>0$ and $A \in\left(\mathcal{G}^{0}\right)^{+}$. Define

$$
H_{t}\left(w, w^{\prime}\right)=A\left(w / t / \sigma_{-t} w^{\prime}\right) 1_{\{\alpha(w)=0\}}
$$

and note that $H_{t}\left(w, \tau_{t} w\right)=A(w)$ if $\alpha(w)=0<t<\beta(w)$. Using the Markov property of each of $Q^{1}$ and $Q_{\nu}$ and the fact that $Q^{1}(\alpha \neq 0)=Q_{\nu}(\alpha \neq 0)=0$, it is easy to check that if $B \in \mathcal{G}_{t+}^{0}$, then

$$
\begin{aligned}
& Q^{1}\left(A 1_{B}\right)=\int_{B} P^{Y_{t}(w)}\left(H_{t}(w, \cdot)\right) Q^{1}(d w) ; \\
& Q_{\nu}\left(A 1_{B}\right)=\int_{B} P^{Y_{t}(w)}\left(H_{t}(w, \cdot)\right) Q_{\nu}(d w) .
\end{aligned}
$$

Thus

$$
\begin{aligned}
Q_{\nu}(J A ; t<\beta) & =Q^{1}(A ; t<\beta) \\
& =\int_{\{t<\beta\}} P^{Y_{t}}(w)\left(H_{t}(w, \cdot)\right) Q^{1}(d w) \\
& =\int_{\{t<\beta\}} J(w) P^{Y_{t}(w)}\left(H_{t}(w, \cdot)\right) Q_{\nu}(d w) \\
& =Q_{\nu}\left(J_{t} A ; t<\beta\right),
\end{aligned}
$$

where $J_{t}$ is a $\mathcal{G}_{t+}^{0}$-measurable version of $d\left(\left.Q^{1}\right|_{\mathcal{G}_{t+}^{0} \cap\{t<\beta\}}\right) / d\left(\left.Q_{\nu}\right|_{\mathcal{G}_{t+}^{0} \cap\{t<\beta\}}\right)$. Since $A \in\left(\mathcal{G}^{0}\right)^{+}$was arbitrary, it follows that $J 1_{\{t<\beta\}} \in\left(\mathcal{G}_{t}^{\nu}\right)^{+} \cap\{t<\beta\}$. Since $Q^{1}$ and $Q_{\nu}$ are carried by $\{\alpha=0\}$ and since $\bigcup_{n=1}^{\infty}\{\alpha=0, \beta>1 / n\}=\{\alpha=0\}$, it follows that $J$ is $\mathcal{G}_{0}^{\nu}$-measurable. Modifying $J$ on a $Q_{\nu}$-null set we may assume that $J \in\left(\mathcal{G}_{0+}^{0}\right)^{+}$and $J<\infty$. Define an optional HRM $\kappa^{1}$ by

$$
\kappa^{1}(d t)=J\left(\tau_{\alpha}\right) 1_{\Lambda^{c}}(t) 1_{\{\alpha \in \mathbf{R}\}} \varepsilon_{\alpha}(d t) .
$$

Let us use (2.5) to check that the Palm measure of $\kappa^{1}$ is $Q^{1}$ :

$$
\begin{aligned}
Q_{m}\left(\int_{\mid 0,1]} F \circ \tau_{t} \kappa^{1}(d t)\right) & =Q_{m}\left(F \circ \tau_{\alpha} J\left(\tau_{\alpha}\right) 1_{\left\{l_{0}=0\right\}}\left(\tau_{\alpha}\right) ; 0<\alpha \leq 1\right) \\
& =Q_{\nu}\left(F J 1_{\left\{l_{0}=0\right\}}\right)=Q^{1}(F) .
\end{aligned}
$$

Thus $\mathbf{P}_{\kappa^{1}}=Q^{1}$. Since $Q^{1}$ is $\sigma$-finite, $\kappa^{1} \in \sigma I\left(\hat{\mathcal{P}}^{m}\right)$ by $(5.11 \mathrm{~b})$. It is easy to check that $\kappa^{1}$ is copredictable. 
(b) Now consider $Q^{2}$. By (3.20), given $Z \in\left(\mathcal{M}^{m}\right)^{+}$there is a function $f_{Z} \in$ $(B \otimes \mathcal{E})^{+}$such that up to $Q_{m}$-evanescence

$$
{ }^{\circ} \hat{p} Z_{t} 1_{\Lambda}(t)={ }^{\hat{p} \circ} Z_{t} 1_{\Lambda}(t)=f_{Z}\left(t, \bar{Y}_{t}\right) .
$$

We claim that if $Z \in\left(I^{m}\right)^{+}$, then for real $a<b$,

$$
\int_{a}^{b} f_{Z}\left(t, \bar{Y}_{0}\right) d t
$$

is $m$ - $\tau$-evanescent. For $(5.16)$, when composed with $\tau_{s}$, may be written as

$$
\int_{s-a}^{s-b} H_{s}(u) d u
$$

where $H_{s}(u, w)=f\left(s-u, \bar{Y}_{s}\right)$. Now for each $u \in \mathbf{R}, H_{s}(u, w)={ }^{\circ} \hat{p} Z_{s-u}\left(\sigma_{u} w\right)$ up to $Q_{m}$-evanescence in $(s, w)$. Thus, for all $u \in \mathbf{R},(s, w) \rightarrow H_{s}(u, w)$ is $Q_{m}$-evanescent. By Fubini's theorem, for $Q_{m}$-a.e. $w$ there is a Lebesgue null set $N_{w} \subset \mathbf{R}$ such that for $u \notin N_{w}$ and all $s \in \mathbf{R}, H_{s}(u, w)=0$. Thus, for $Q_{m}$-a.e. $w$ the integral in (5.17) vanishes for all $s \in \mathbf{R}$. Recalling that (5.17) is (5.16) composed with $\tau_{s}$, it follows that the integral in (5.16) is $m$ - $\tau$-evanescent. Since $Q^{2}$ charges no $m$ - $\tau$-evanescent set we may unambiguously define a measure on $\mathcal{M}^{m}$ by

$$
M(Z)=Q^{2}\left(\int_{\mathbf{R}} f_{Z}\left(t, \bar{Y}_{0}\right) d t\right), \quad Z \in\left(\dot{\mathcal{M}}^{m}\right)^{+} .
$$

(Because of the preceding discussion the right side of (5.18) does not depend on the particular choice of $f_{Z}$. .) Clearly $M(Z)=0$ if $Z \in I^{m}$ and $M(Z)=M\left({ }^{\hat{p}} Z\right)=$ $M\left({ }^{\circ} Z\right)$ if $Z \in\left(\mathcal{M}^{m}\right)^{+}$; of course $M$ is carried by $\Lambda$. Since a version of $f_{\Sigma_{-s} Z}$ is given by $f_{Z}(\cdot+s, \cdot)$ it follows that $\Sigma_{s} M=M$ for all $s \in \mathbf{R}$. We claim that $M$ is $\sigma$-finite on $\left(0^{0} \cap \hat{p}^{0}\right)$. Indeed with $q$ and $\Omega_{q}$ as in $(2.8)$, since $Q^{2}$ is carried by $\left\{l_{0}>0\right\} \subset \Omega_{q}$ (see (3.6)), we deduce from (2.8) that $Q^{2}=P^{\rho}$ where $\rho$ is the $\sigma$-finite law of $\bar{Y}_{0}$ under $Q^{2}$. Choose $g>0$ and Lebesgue integrable over $\mathbf{R}$, and $f \in \mathcal{E}^{+}$with $f>0$ and $\rho(f)<\infty$. Set $Z_{t}=g(t) f\left(\bar{Y}_{t}\right)$ so that $Z \in\left(0^{0} \cap \hat{p}^{0}\right)^{+}$ and $Z>0$ on $\Lambda$. We have $M(Z)=\lambda(g) \rho(f)<\infty$ so that $M$ is $\sigma$-finite as claimed. By (5.3b) $M$ is the Doleans measure of a unique HRM $\kappa^{2}$ in $\sigma I\left(0^{0} \cap \hat{p}^{0}\right)$ which is optional, copredictable, and carried by $\Lambda$. It is easy to check that $\mathbf{P}_{\kappa^{2}}=Q^{2}$.

Combining (a) and (b) we obtain the desired HRM with Palm measure $Q_{\mu}$ by setting $\kappa=\kappa^{1}+\kappa^{2}$. The uniqueness assertion is obvious from the construction of $\kappa$.

(5.19) REMARK. The $\sigma$-finiteness of $\int_{0}^{\infty} \mu_{t} d t$ was used only in showing that $Q^{2}=P^{\rho}$ and that each $\mu_{t}$ is $\sigma$-finite. If these facts were known by other means then the above construction would still yield a unique optional copredictable HRM with Palm measure $Q_{\mu}$.

We now consider several refinements of (5.15). Firstly, the criterion for a measure on $\left(W, \mathcal{G}^{0}\right)$ to be a Palm measure can be simplified considerably when the entrance law $\mu$ takes the special form $\mu_{t}=\rho P_{t}$ where $\rho$ is a measure on $(E, \mathcal{E})$. For the next result let $m=\pi U+\gamma$ be the Riesz decomposition of $m$ into potential and harmonic components. See $(3.7)$ of $[\mathbf{1 9}]$. 
(5.20) COROLlaRY. Let $\rho$ be a $\sigma$-finite measure on $(E, \mathcal{E})$. Then $P^{\rho}$ is the Palm measure of a unique optional, copredictable $H R M \kappa$ if and only if $\rho(B)=0$ whenever $B \in \mathcal{E}$ satisfies (i) $\pi(B)=0$, (ii) $B$ is m-polar.

PROOF. In view of (5.15) and (5.19) we must check that the stated condition on $\rho$ is equivalent to the statement that $P^{\rho}$ charges no $m$ - $\tau$-evanescent set. Let $m=$ $m_{p}+m_{i}$ be the decomposition of $m$ into purely excessive and invariant components; thus $m_{p}=\int_{0}^{\infty} \nu_{t} d t$ where $\nu=\left(\nu_{t}\right)$ is an entrance law. One checks that the Riesz decomposition of $m_{p}$ into potential and harmonic components is given by $m_{p}=$ $\pi U+\gamma^{\prime}$ (recall that $\pi U$ is the potential part of $m$ ). Clearly $\gamma^{\prime}$ is purely excessive so that $\gamma^{\prime}=\int_{0}^{\infty} \nu_{t}^{\prime} d t$ for some entrance law $\nu^{\prime}=\left(\nu_{t}^{\prime}\right)$. Moreover $\nu_{t}=\pi P_{t}+\nu_{t}^{\prime}$ so that $Q_{\nu}=P^{\pi}+Q_{\nu^{\prime}} \geq P^{\pi}$.

Now suppose that $\rho(B)=0$ whenever $B$ is a Borel $m$-polar set with $\pi(B)=0$. Let $A \in \mathcal{G}^{0}$ be $m$ - $\tau$-evanescent; that is $A$ is $m-\tau$-polar and $Q_{\nu}(A)=0$. Consider $B=\left\{x: P^{x}(A)>0\right\} \in \mathcal{E}$. Since $0=Q_{\nu}(A) \geq P^{\pi}(A)$, we have $\pi(B)=0$. Since $A$ is $m$ - $\tau$-polar, $B$ is $m$-polar. Thus $\rho(B)=0$ and so $P^{\rho}(A)=\int \rho(d x) P^{x}(A)=0$. That is, $P^{\rho}$ does not charge the (arbitrary) $m$ - $\tau$-evanescent set $A$.

Conversely, suppose that $P^{\rho}(A)=0$ whenever $A \in \mathcal{G}^{0}$ is $m$ - $\tau$-evanescent. Let $B \in \mathcal{E}$ be $m$-polar with $\pi(B)=0$. Consider the event $A=\left\{Y_{0+} \in B\right\} \cap \Omega_{q}$, where $q$ and $\Omega_{q}$ are as in (2.8) (relative to $m_{p}$ ). Since $B$ is $m$-polar, $A$ is $m$ - $\tau$-polar. Also,

$$
\begin{aligned}
Q_{\nu}(A) & =P^{\pi}(A)+Q_{\nu^{\prime}}(A) \\
& =\pi(B)+Q_{\nu^{\prime}}\left(Y_{0+} \in B ; \Omega_{q}\right)=0
\end{aligned}
$$

where for the second equality we have used the fact that $P^{\pi}$ is carried by $\Omega_{q}$, and for the third the fact that $Q_{\nu^{\prime}}\left(\Omega_{q}\right)=0$ since $\int_{0}^{\infty} \nu_{t}^{\prime} d t$ is harmonic (see $\S 3$ of $[\mathbf{1 9}]$ ). Thus $A$ is $m$ - $\tau$-evanescent and so $P^{\rho}(A)=\rho(B)=0$.

The reader familiar with the work of Azema will recognize (5.20) (and, a fortiori, (5.15)) as an extension of Théorème 1, p. 491, of [2]. See Remark (5.30) for more on this point.

Now suppose that $\kappa$ is an optional HRM which is carried by $\Lambda$. Define the characteristic measure of $\kappa$ by

$$
\rho_{\kappa}(f)=\mathbf{P}_{\kappa}\left(f\left(\bar{Y}_{0}\right)\right) .
$$

Recalling (3.22), it is obvious that $\rho_{\kappa}$ charges no $m$-polar set. Although $\rho_{\kappa}$ need not be $\sigma$-finite the argument of (5.13) remains valid and we have $\rho_{\kappa} P_{t}=\mu_{t}^{\kappa}, t>0$, where $\mu_{t}^{\kappa}$ is as defined just prior to (5.13). If, additionally, $\kappa$ is a diffuse HRM (i.e., if for $Q_{m}$-a.e. $w, \kappa(w, \cdot)$ is a diffuse measure on $\mathbf{R}$ ), then $\rho_{\kappa}$ charges no Borel $m$-semipolar set. Here, in view of $\S 6$ of [29], we say that $B \in \mathcal{E}$ is $m$-semipolar provided $\left\{t: Y_{t} \in B\right\}$ is countable for $Q_{m}$-a.e., $w$. Conversely we have the following.

(5.22) COROLlaRY. Let $\rho$ be a $\sigma$-finite measure on $(E, \mathcal{E})$ which charges no $m$-polar set. Then there exists a unique optional copredictable HRM $\kappa$, which is carried by $\Lambda$, with characteristic measure $\rho$. Moreover, $\kappa$ is diffuse if and only if $\rho$ charges no m-semipolar set.

PROOF. If $\rho$ charges no $m$-polar set, then by $(5.20) P^{\rho}$ is the Palm measure of a unique optional copredictable HRM $\kappa$. Indeed, arguing as in the proof of $(5.20)$ one sees that $P^{\rho}$ charges no $m$ - $\tau$-polar set; by $(5.11 \mathrm{c}), \kappa$ is carried by $\Lambda$. It now follows easily that $\rho$ is the characteristic measure of $\kappa$. 
Half of the assertion concerning the diffuseness of $\kappa$ follows from the discussion preceding the corollary. Conversely, suppose that $\rho=\rho_{\kappa}$ charges no $m$-semipolar Borel set. Let $\kappa=\kappa^{c}+\kappa^{d}$ be the decomposition of $\kappa$ into diffuse (=continuous) and discrete components. More precisely, let $J_{t}=\kappa\{t\}$ and note that $\left(J_{t}\right) \in$ $\left(O^{m} \cap \hat{p}^{m}\right)^{+}$and that $\left(J_{t}\right)$ is homogeneous and vanishes off $\Lambda$. By $(3.21),\left(J_{t}\right)$ is $Q_{m}$-indistinguishable from a process of the form $j\left(\bar{Y}_{t}\right)$ where $j \in \mathcal{E}^{+}$. The discrete component $\kappa^{d}$ is then $\sum_{s \in \mathbf{R}} \kappa\{s\} \varepsilon_{s}$ and so is indistinguishable from the $\operatorname{HRM} \sum_{s \in \mathbf{R}} j\left(\bar{Y}_{s}\right) \varepsilon_{s}$. Now for each $w, \kappa(w, \cdot)$ is a countable sum of finite measures and as such can have at most countably many atoms. Thus

$$
0=Q_{m}\left(\left\{t: \bar{Y}_{t} \in\{j>0\} \text { is uncountable }\right\}\right) .
$$

Since $Y_{t}$ and $\bar{Y}_{t}$ can differ only at $t=\alpha$, it follows that $\{j>0\}$ is $m$-semipolar. Thus $\rho(\{j>0\})=0$ and consequently $\kappa^{d}$ is $Q_{m}$-indistinguishable from the zero measure. Thus $\kappa=\kappa^{c}$ is diffuse.

Our final refinement of (5.15) is a "perfection" theorem. Let us first set down our notion of perfection for a HRM.

(5.23) DEFInition. Let $\mathcal{G}^{*}$ denote the universal completion of $\mathcal{G}^{0}$. We say that a HRM $\kappa$ is perfect provided

(i) $\kappa$ is a kernel from $\left(W, \mathcal{G}^{*}\right)$ to $(\mathbf{R}, B)$;

(ii) $\Sigma_{s} \kappa(w, \cdot)=\kappa(w, \cdot), \forall w \in W, \forall s \in \mathbf{R}$;

(iii) $\kappa\left(b_{s} w, B\right)=\kappa(w, B \cap[s+\infty[), \forall w \in W, \forall s \in \mathbf{R}, \forall B \in B$;

(iv) $\kappa\left(k_{s} w, B\right)=\kappa(w, B \cap]-\infty, s[), \forall w \in W, \forall s \in \mathbf{R}, \forall B \in B$.

As we shall see, condition (iii) (resp. (iv)) amounts to the statement that $\kappa$ is $\left(\hat{\mathcal{G}}_{t+}^{*}\right)$-predictable (resp. $\left(\mathcal{G}_{t+}^{*}\right)$-optional). Here $\hat{\mathcal{G}}_{t}^{*}=b_{t}^{-1} \mathcal{G}^{*}, \mathcal{G}_{t}^{*}=\kappa_{t}^{-1} \mathcal{G}^{*}$. For this we set down criteria for optionality and copredictability, in terms of "adaptedness" conditions (such as (iii) and (iv) above). The first criterion (5.24) is simply a translation into our context of a standard criterion for predictability (cf. [12, Chapter VI, T57, T59]). The second criterion (5.25), and the idea for its proof, is borrowed from Mitro [38].

(5.24) Proposition. Let $\kappa$ be a $R M$ in $\sigma I\left(\hat{p}^{m}\right)$ and let $Z \in \hat{p}^{m}$ with $Z>0$ and $\langle Z, \kappa\rangle<\infty$. For each $r \in \mathbf{R}$ define a process $C^{r}$ by

$$
C_{t}^{r}=1_{]-\infty, r[}(t) \int_{[t, r]} Z_{s} \kappa(d s), \quad t \in \mathbf{R} .
$$

Then $\kappa$ is $Q_{m}$-copredictable if and only if $C^{r} \in \hat{p}^{m}$ for each $r \in \mathbf{R}$.

(5.25) PROPOSITION. Let $\kappa$ be a $R M$ in $\sigma I\left(O^{m}\right)$ which is carried by $\Lambda$. Then $\kappa$ is $Q_{m}$-optional if and only if $\kappa(\cdot, B) \in \mathcal{G}_{t}^{m}$ for all $t \in \mathbf{R}$ and all $\left.\left.B \in B \cap\right]-\infty, t\right]$.

ProOF. Replacing $\kappa$ by $Z * \kappa$ where $Z \in\left(O^{m}\right)^{+}$is strictly positive with $\langle Z, \kappa\rangle<$ $\infty$, we may assume that $Q_{m}(\kappa(\mathbf{R}))<\infty$. In particular, $\kappa(\mathbf{R})<\infty$ a.e., $Q_{m}$.

(a) Assume that $\kappa$ is $Q_{m}$-optional. Fix reals $u<v$ and $H \in \mathcal{G}^{0}$ with $0 \leq H \leq 1$ and $Q_{m}(H)<\infty$. Let $\left(H_{t}\right)$ be a rc version of the martingale $Q_{m}\left(H \mid \mathcal{G}_{t}^{m}\right)$. The processes

$$
Z_{t}=1_{\mathrm{\jmath} u, v]}(t) \cdot H, \quad \tilde{Z}_{t}=1_{\mathrm{\jmath} u, v]}(t) H_{v}
$$

have the same $Q_{m}$-optional projection, namely $1_{\Lambda}(t) 1_{] u, v]}(t) H_{t}$. Since $\kappa$ is optional we may use (5.6) to compute

$$
\left.\left.\left.\left.Q_{m}(H \cdot \kappa] u, v\right]\right)=\langle Z, \kappa\rangle=\left\langle{ }^{\circ} Z, \kappa\right\rangle=\left\langle^{\circ}(\tilde{Z}), \kappa\right\rangle=\langle\tilde{Z}, \kappa\rangle=Q_{m}\left(H_{v} \kappa\right] u, v\right]\right) .
$$


Thus $\kappa] u, v] \in \mathcal{G}_{v}^{m}$. It now follows easily that $\kappa(\cdot, B) \in \mathcal{G}_{t}^{m}$ whenever $B \in B \cap$ ]$-\infty, t]$.

(b) Conversely, assume that $\kappa(B) \in \mathcal{G}_{t}^{m}$ whenever $\left.\left.B \in B \cap\right]-\infty, t\right]$. To prove that $\kappa$ is $Q_{m}$-optional it suffices to show that $\langle Z, \kappa\rangle=\left\langle{ }^{\circ} Z, \kappa\right\rangle$ for each rc $Z \in$ $b\left(\mathcal{M}^{m}\right)^{+}$which vanishes on $\llbracket \beta,+\infty \llbracket$. Fix such a $Z$ and recall from (3.19) that ${ }^{\circ} Z$ is $\mathrm{rc}$ on $\Lambda$. For $k \in \mathbf{Z}$ and $n \in \mathbf{N}$ let $\left.A(n, k)=\kappa] k 2^{-n},(k+1) 2^{-n}\right], Z(n, k)=$ $Z_{(k+1) 2^{-n}},{ }^{\circ} Z(n, k)={ }^{\circ} Z_{(k+1) 2^{-n}}$. Since $\kappa(\mathbf{R})<\infty$ a.e., $Q_{m}$, we have by bounded convergence

$$
\int_{\mathbf{R}} Z_{t} \kappa(d t)=\lim _{n \rightarrow \infty} \sum_{k \in \mathbf{Z}} Z(n, k) A(n, k), \quad \text { a.e. } Q_{m},
$$

the sums on the right being bounded by the integrable random variable $\|Z\| \kappa(\cdot, \mathbf{R})$. An analogous statement holds for $\int_{\mathbf{R}}{ }^{\circ} Z_{t} \kappa(d t)$. But $A(n, k) \in \mathcal{G}_{(k+1) 2^{-n}}^{m}$ and so

$$
\begin{aligned}
Q_{m}(Z(n, k) A(n, k)) & =Q_{m}\left(Z(n, k) A(n, k) ;(k+1) 2^{-n} \in \Lambda\right) \\
& =Q_{m}\left({ }^{\circ} Z(n, k) A(n, k) ;(k+1) 2^{-n} \in \Lambda\right) \\
& =Q_{m}\left({ }^{\circ} Z(n, k) A(n, k)\right),
\end{aligned}
$$

where for the first equality we have used the fact that $Z=0$ on $\llbracket \beta,+\infty \llbracket$, that

$$
Q_{m}\left((k+1) 2^{-n} \in \llbracket \alpha, \beta \llbracket \backslash \Lambda\right) \leq Q_{m}\left((k+1) 2^{-n}=\alpha\right)=0,
$$

and that $A(n, k)=0$ if $(k+1) 2^{-n}<\alpha$. The dominated convergence theorem now yields

$$
\begin{aligned}
\langle Z, \kappa\rangle & =\lim _{n \rightarrow \infty} \sum_{k} Q_{m}(Z(n, k) A(n, k)) \\
& =\lim _{n \rightarrow \infty} \sum_{k} Q_{m}\left({ }^{\circ} Z(n, k) A(n, k)\right)=\left\langle{ }^{\circ} Z, \kappa\right\rangle,
\end{aligned}
$$

as required.

(5.26) COROLlaRY. Let $\kappa$ be a HRM carried by $\Lambda$. Suppose that $\kappa \in \sigma I\left(\hat{p}^{m}\right) \cap$ $\sigma I\left(O^{m}\right)$ and that $\kappa$ is perfect (in the sense of (5.23)). Then $\kappa$ is $Q_{m}$-optional and $Q_{m}$-copredictable.

Proof. (a) Fix $t \in \mathbf{R}$ and $B \in]-\infty, t]$. Choose any $s>t$. Then $\kappa(w, B)=$ $\kappa\left(k_{s} w, B\right)$ by $(5.23)$ (iv) and so $\kappa(\cdot, B) \in k_{s}^{-1} \mathcal{G}^{*}$. Thus $\kappa(\cdot, B) \in \bigcap_{s>t} k_{s}^{-1} \mathcal{G}^{*} \subset \mathcal{G}_{t}^{m}$. Thus $\kappa$ is $Q_{m}$-optional by (5.25).

(b) Choose $Z>0$ in $\hat{P}$ with $\langle Z, \kappa\rangle<\infty$ and define $C^{r}$ as in Proposition (5.24). Then by (5.23)(iii) and (3.1c)

$$
C_{t}^{r}=\left(\int_{|-\infty, r|} Z_{s} \kappa(d s)\right) \circ b_{t}, \quad t \in \mathbf{R} .
$$

It follows from (3.1b) that $C^{r} \in \hat{P}^{m}$. Thus, by (5.24), $\kappa$ is $Q_{m}$-copredictable.

Here is our result on the perfection of HRM's.

(5.27) THEOREM. Let $\kappa$ be an optional, copredictable HRM. Then there exists a perfect HRM $\bar{\kappa}$ which is $Q_{m}$-indistinguishable from $\kappa$. 
ProOF. First decompose $\kappa$ as $\kappa^{1}+\kappa^{2}=1_{\Lambda^{c}} * \kappa+1_{\Lambda} * \kappa$. Since $\kappa$ is $Q_{m}$-optional $\kappa^{1}=J_{\alpha} 1_{\Lambda^{c}} 1_{\{\alpha \in \mathbf{R}\}} \varepsilon_{\alpha}$, where $J_{\alpha} \in\left(\mathcal{G}_{\alpha}^{m}\right)^{+}$is finite a.e. $Q_{m}$ and satisfies

$$
Q_{m}\left(J_{\alpha} \neq J_{\alpha} \circ \sigma_{s}\right)=0, \quad \forall s \in \mathbf{R}
$$

Arguing as in part (a) of the proof of (5.15), there is a finite valued $J \in\left(\mathcal{G}_{0}^{0}\right)^{+}$ such that $Q_{m}\left(J_{\alpha} \neq J\left(\tau_{\alpha}\right) ; \alpha \in \mathbf{R}, l_{\alpha}=0\right)=0$. The HRM $\bar{\kappa}$ defined by $\bar{\kappa}^{1}=$ $J\left(\tau_{\alpha}\right) 1_{\Lambda^{c}} 1_{\{\alpha \in \mathbf{R}\}} \varepsilon_{\alpha}$ is $Q_{m}$-indistinguishable from $\kappa^{1}$, and $\bar{\kappa}^{1}$ is easily seen to be perfect.

Next, decompose $\kappa^{2}=1_{\Lambda} * \kappa$ into diffuse and discrete parts, say $\kappa^{2}=\kappa^{c}+\kappa^{d}$. As in the proof of (5.22), $\kappa^{d}$ is $Q_{m}$-indistinguishable from a HRM of the form

$$
\bar{\kappa}^{d}=\sum_{s \in \mathbf{R}} j\left(\bar{Y}_{s}\right) \varepsilon_{s}
$$

where $j \in \mathcal{E}^{+}$and $\{j>0\}$ is $m$-semipolar. Again, it is easy to check that (5.28) defines a perfect HRM.

Finally, let $\rho$ denote the characteristic measure of $\kappa^{c}$. Since $\kappa^{c}$ is carried by $\rrbracket \alpha, \beta \llbracket$ we have

$$
\rho(f)=Q_{m}\left(\int_{0}^{1} f \circ Y_{t} \kappa^{c}(d t)\right), \quad f \in \mathcal{E}^{+},
$$

and $\rho$ charges no $m$-semipolar set. We are now going to invoke Theorem 1.7 of [17]. Because of $(4.15)$, the hypothesis $1.7 \mathrm{~A}$ of [17] is satisfied. For the moment assume that $\rho(E)<\infty$. Dynkin and Getoor in [17] assume also a transience hypothesis that need not be satisfied by $\rho$ (relative to $m$ and $\left(P_{t}\right)$ ). But consider the 1-subprocess $X^{1}$ with semigroup $P_{t}^{1}=e^{-t} P_{t}$. Clearly $m$ is excessive for $\left(P_{t}^{1}\right)$, and $X$ and $X^{1}$ have identical classes of $m$-semipolar sets. Thus, $\rho$ charges no $m$ semipolars for $X^{1}$. Choose $q \in \mathcal{E}^{+}$with $0<q \leq 1$. Then $\rho U^{1} q \leq \rho(E)<\infty$ and $0<U^{1} q \leq 1$. Therefore the transience hypothesis stated at the beginning of subsection 1.7 of $[\mathbf{1 7}]$ is satisfied by $\rho$, relative to $m$ and $\left(P_{t}^{1}\right)$. Consequently, if $Q_{m}^{1}$ denotes the Kuznetsov measure associated with $m$ and $\left(P_{t}^{1}\right)$, then $[\mathbf{1 7}, 1.7]$ yields a diffuse perfect $\mathrm{HRM} \bar{\kappa}^{c}$ such that

$$
Q_{m}^{1}\left(\int_{0}^{1} f\left(Y_{t}\right) \bar{\kappa}^{c}(d t)\right)=\rho(f), \quad f \in \mathcal{E}^{+}
$$

Now an easy comparison of finite dimensional distributions yields the relation

$$
Q_{m}^{1}(F)=Q_{m}\left(\iint_{u<v} F \circ k_{v} \circ b_{u} 1_{\{\alpha<v, u<\beta\}} e^{-(v-u)} d u d v\right)
$$


whenever $F \in\left(\mathcal{G}^{*}\right)^{+}$. We use (5.29) to compute the characteristic measure of $\bar{\kappa}^{c}$ relative to $Q_{m}$ : if $g \in B^{+}$with $\lambda(g)=1$, then

$$
\begin{aligned}
\rho(f) & =Q_{m}^{1}\left(\int_{\mathbf{R}} g(t) f\left(Y_{t}\right) \bar{\kappa}^{c}(d t)\right) \\
& =Q_{m}\left(\iint_{u<v} \int_{u}^{v} g(t) f\left(Y_{t}\right) \bar{\kappa}^{c}(d t) e^{-(v-u)} d u d v\right) \\
& =Q_{m}\left(\int_{\mathbf{R}} d u \int_{u}^{\infty} g(t) f\left(Y_{t}\right) \bar{\kappa}^{c}(d t) \int_{t}^{\infty} e^{-(v-u)} d v\right) \\
& =Q_{m}\left(\int_{\mathbf{R}} d u \int_{u}^{\infty} g(t) e^{-(t-u)} f\left(Y_{t}\right) \bar{\kappa}^{c}(d t)\right) \\
& =Q_{m}\left(\int_{\mathbf{R}} g(t) f\left(Y_{t}\right) \bar{\kappa}^{c}(d t)\right) \\
& =\rho_{\bar{\kappa}^{c}}(f) .
\end{aligned}
$$

That is, $\rho$ is also the characteristic measure of $\bar{\kappa}^{c}$, relative to $Q_{m}$. As in the proof of (5.15), $\bar{\kappa}^{c} \in \sigma I\left(O^{m}\right) \cap \sigma I\left(\hat{p}^{m}\right)$; by Corollary (5.26), $\bar{\kappa}^{c}$ is $Q_{m}$-optional and $Q_{m^{-}}$ copredictable. By the uniqueness part of $(5.22), \bar{\kappa}^{c}$ and $\kappa^{c}$ are $Q_{m}$-indistinguishable.

In general $\rho$ is only $\sigma$-finite but we can decompose $\rho$ as $\sum \rho_{n}$ where each $\rho_{n}$ is a finite measure charging no $m$-semipolar set. By the discussion above, for each $n$ there is a diffuse, perfect $\mathrm{HRM} \bar{\kappa}_{n}$ with characteristic measure $\rho_{n}$. Set $\bar{\kappa}^{c}=\sum_{n} \bar{\kappa}_{n}$ so that $\bar{\kappa}^{c}$ is a diffuse, perfect HRM with characteristic measure $\rho$. As before, $\bar{\kappa}^{c}$ is optional, copredictable and indistinguishable from $\kappa^{c}$. Setting $\bar{\kappa}=\bar{\kappa}^{1}+\bar{\kappa}^{d}+\bar{\kappa}^{c}$ we have obtained the desired perfect version of $\kappa$.

(5.30) REMARK. Let us return to the situation of Corollary (5.20). Assume that $m$ is a potential, say $m=\pi U$ and that $\rho$ is a finite measure on $(E, \mathcal{E})$ such that $\rho(B)=0$ whenever $B$ is a Borel $m$-polar set with $\pi(B)=0$. (In the terminology of Azema [2], such a set $B$ is " $\pi$-evanescent".) Let $\kappa$ be the perfect HRM promised by (5.20) and (5.27). Define an increasing process $\left(A_{t}\right)$ on $\Omega=\left\{\alpha=0, Y_{\alpha+}\right.$ exists in $E\} \cup\{[\Delta]\}$ by

$$
A_{t}(w)=\kappa(w,[0, t]), \quad t \geq 0, w \in \Omega .
$$

Because of (5.23iv), $A_{t} \in \bigcap_{s>t}\left[\left(k_{s}^{-1} \mathcal{G}^{*}\right) \cap \Omega\right], \forall t \geq 0$. Because of (5.23)(iii), since $\theta_{t}=\tau_{t}=b_{0} \sigma_{t}$ on $\Omega$,

$$
A_{t+s}(w)=A_{t-}(w)+A_{s}\left(\theta_{t} w\right), \quad t \geq 0, s>0, w \in \Omega
$$

where $A_{0-}(w)=0$ by convention. Since $\kappa$ is carried by $\llbracket \alpha, \beta \llbracket$, the measure $d A_{t}(w)$ is carried by $\llbracket 0, \varsigma \llbracket$. In Azema's terminology, $\left(A_{t}\right)$ is a "fonctionelle droite". Moreover $\left(A_{t}\right)$ is the representing functional for $\rho$ relative to $P^{\pi}$ :

$$
\rho(f)=P^{\pi}\left(\int_{0}^{\infty} f\left(X_{t}\right) d A_{t}\right), \quad f \in \mathcal{E}^{+} .
$$

Thus (5.20) generalizes Théorème 1, p. 491 of [2] as claimed previously. To see (5.32) use (2.5) $\left(m=\pi U=\int_{0}^{\infty} \pi P_{t} d t\right)$ and (5.12) to compute: for $g \in B^{+}$with 
$\int_{\mathbf{R}} g(t) d t=1$

$$
\begin{aligned}
\rho(f) & =\int_{\mathbf{R}} g(t) d t P^{\rho}\left(f \circ Y_{0}\right) \\
& =Q_{m}\left(\int_{\mathbf{R}} g(t) f \circ Y_{t} \kappa(d t)\right) \\
& =Q_{m}\left(\int_{[0,+\infty[} g(\alpha+u) f\left(Y_{u+} \circ \tau_{\alpha}\right) \kappa\left(\tau_{\alpha}, d u\right)\right) \\
& =\int_{\mathbf{R}} d t P^{\pi}\left(\int_{[0,+\infty[} g(t+u) f\left(X_{u}\right) \kappa(d u)\right) \\
& =P^{\pi}\left(\int_{[0,+\infty[} f\left(X_{u}\right) d A_{u}\right) .
\end{aligned}
$$

6. A weak order for excessive measures. Theorem (5.15) may be regarded as a Skorohod representation theorem of sorts. For if $\xi$ is purely excessive then we have $\xi=\int_{0}^{\infty} \mu_{t} d t$ where $\mu=\left(\mu_{t}\right)$ is an entrance law with Kuznetsov measure $Q_{\mu}$. Suppose that $Q_{\mu}$ charges no $m$ - $\tau$-evanescent set. Then (5.15) and (5.27) guarantee the existence of a perfect, optional, copredictable HRM $\kappa$ such that $\mathbf{P}_{\kappa}=Q_{\mu}$. Now by (2.6) we have

$$
Q_{\xi}(F)=\int_{\mathbf{R}} Q_{\mu}\left(F \circ \sigma_{-t}\right) d t, \quad F \in\left(\mathcal{G}^{0}\right)^{+} .
$$

But $\tau_{t}=b_{0} \sigma_{t}$ and $\mathbf{P}_{\kappa}=b_{0}\left(\mathbf{P}_{\kappa}\right)$, since $\mathbf{P}_{\kappa}$ is carried by $\{\alpha=0\}$. Using (5.12) with $\mathbf{P}_{\kappa}=Q_{\mu}$ we deduce from (6.1) that

$$
Q_{\xi}(F)=Q_{m} \int_{\mathbf{R}} F \circ b_{t} \kappa(d t), \quad F \in\left(\mathcal{G}^{0}\right)^{+} .
$$

That is, $\left(Y_{t}\right)$ under $Q_{\xi}$ is the same as $\left(Y_{t}\right)$ under $Q_{m}$ "birthed" at rate $\kappa(d t)$. A stopping time version of (6.2), which is more in the spirit of Skorohod stopping, may be found in (6.36) below.

Let $\hat{u}(x)=\hat{P}^{x}(\kappa]-\infty, 0[)$ where $\left(\hat{P}^{x}\right)$ is the moderate Markov dual for $\left(P^{x}\right)$ relative to $m$. Since $\kappa]-\infty, 0\left[\in \mathcal{G}^{*}(]-\infty, 0[)\right.$ we have $\left.\kappa\right]-\infty, 0\left[\circ \hat{\tau}_{0}=\kappa\right]-\infty, 0[$. Taking $F=f\left(Y_{0}\right)$ in (6.2) we may therefore compute

$$
\begin{aligned}
\xi(f)=Q_{\xi}\left(f\left(Y_{0}\right)\right) & =Q_{m}\left(f\left(Y_{0}\right) \kappa\right]-\infty, 0[) \\
& =Q_{m}\left(f\left(\bar{Y}_{0}\right) \kappa\right]-\infty, 0\left[\circ \hat{\tau}_{0} ; 0<\beta\right) \\
& =Q_{m}\left(f\left(\bar{Y}_{0}\right) \hat{u}\left(\bar{Y}_{0}\right) ; 0<\beta\right) \\
& =Q_{m}\left((f \hat{u})\left(Y_{0}\right)\right)=m(f \hat{u}) .
\end{aligned}
$$

We have used the fact that $Q_{m}\left(Y_{0} \neq \bar{Y}_{0}\right)=0$ in the above computation. Thus $\hat{u}$ is a version of $d \xi / d m$.

Conversely, if $\xi$ is purely excessive and $\xi<<m$, then (4.15) yields a version $\phi$ of $d \xi / d m$ such that $t \rightarrow \phi\left(\bar{Y}_{t}\right)$ is rc on $\Lambda$. Moreover, $\left(\phi\left(\hat{X}_{t}\right): t>0\right)$ is a supermartingale under $\hat{P}^{x}$ for each $x \in E$. Actually, in a sense that can be made precise, $\left(\phi\left(\bar{Y}_{t}\right):-\infty<t<\beta\right)$ is a supermartingale under $Q_{m}$, relative to the backward filtration $\left(\hat{\mathcal{G}}_{t}^{m}\right)$. One then expects that a representation of the form (6.2) will hold 
precisely when $\phi\left(\bar{Y}_{t}\right)$ is a "class D" supermartingale (in an appropriate sense). We shall see that this is the case in Theorem (6.27). The reader familiar with the work of Azema [2] will recognize (6.2) as dual of sorts to his killing formula for $h$-transforms.

Since $b_{t}^{-1}\{\alpha=-\infty\}=\{\alpha \vee t=-\infty\}=\varnothing$, to obtain a representation of the form (6.2) for an excessive measure with nonzero invariant part we must allow $\kappa$ to charge $\{-\infty\}$ when $\alpha=-\infty$. This explains the presence of the second term on the right side of $(6.4)$ below.

(6.3) DEFinition. Let $\xi$ and $m$ be excessive measures. We say that $\xi$ is weakly dominated by $m$, and write $\xi \leftarrow m$, provided there is an optional, copredictable HRM $\kappa$ and a finite-valued random variable $J \in\left(\mathcal{G}_{\alpha}^{m}\right)^{+}$with $J=J \circ \sigma_{s}, \forall s \in \mathbf{R}$, such that

$$
Q_{\xi}(F)=Q_{m} \int_{\mathbf{R}} F \circ b_{t} \kappa(d t)+Q_{m}(F J ; \alpha=-\infty), \quad F \in\left(\mathcal{G}^{0}\right)^{+} .
$$

(6.5) REMARKS. (a) Because of (5.27), the HRM $\kappa$ in (6.3) can be taken to be perfect.

(b) Let $\xi=\xi_{p}+\xi_{i}$ be the decomposition of $\xi$ into purely excessive and invariant parts. Then the first term on the right in $(6.4)$ is $Q_{\xi_{p}}(F)$ while the second is $Q_{\xi_{i}}(F)$. Evidently $\xi \leftarrow m$ iff $\xi_{p} \leftarrow m$ and $\xi_{i} \leftarrow m$.

Our first characterization of the weak order $\leftarrow$ on Exc is an easy consequence of (5.15).

(6.6) TheOREM. Let $\xi$ and $m$ be excessive measures. Let $\xi=\xi_{i}+\xi_{p}=$ $\xi_{i}+\int_{0}^{\infty} \mu_{t} d t$ be the decomposition of $\xi$ into invariant and purely excessive parts. Let $Q_{\mu}$ denote the Kuznetsov measure for the entrance law $\mu=\left(\mu_{t}\right)$. Then $\xi \leftarrow m$ iff

(i) $Q_{\mu}$ charges no $m-\tau$-evanescent set; and

(ii) $Q_{\xi_{i}}<<Q_{m}$.

In this case $\kappa$ is as guaranteed by (5.15) and $J$ is a suitable version of $d Q_{\xi_{i}} / d Q_{m}$.

PROOF. In view of (5.15), and the definition (6.3), the only part of (6.6) that is not obvious is the assertion that if $Q_{\xi_{i}}<<Q_{m}$ then $d Q_{\xi_{i}} / d Q_{m}$ can be chosen to be $\mathcal{G}_{\alpha}^{m}$-measurable and $\left(\sigma_{t}\right)$-invariant. To see this decompose $m=m_{i}+m_{p}$ into invariant and purely excessive parts. Clearly $Q_{\xi_{i}} \perp Q_{m_{p}}$ and so $Q_{\xi_{i}}<<Q_{m}$ iff $Q_{\xi_{i}}<<Q_{m_{i}}$. Let $J^{\circ}$ be any $\mathcal{G}^{0}$-measurable version of $d Q_{\xi_{i}} / d Q_{m_{i}}$ and use the obvious modification of the proof of $(5.15)$ to show that $J^{\circ}$ is $\mathcal{G}_{\alpha}^{m_{i}}$-measurable. Since $Q_{\xi_{i}}$ and $Q_{m_{i}}$ are stationary we have $J^{\circ}=J^{\circ} \circ \sigma_{s}$ a.e. $Q_{m}$ for all $s \in \mathbf{R}$. If we set

$$
G_{1}=\left\{w: J^{\circ}(w)=J^{\circ}\left(\sigma_{s} w\right) \text { for Lebesgue a.e. } s \in \mathbf{R}\right\},
$$

then $G_{1} \in \mathcal{G}^{0}$ and a Fubini argument shows that $Q_{m}\left(W \backslash G_{1}\right)=0$. Thus $0=$ $Q_{m}\left(W \backslash G_{1}\right)=Q_{m}\left(\sigma_{t}^{-1}\left(W \backslash G_{1}\right)\right)$ for any $t \in \mathbf{R}$. A second application of Fubini's theorem shows that if

$$
G_{2}=\left\{w: \sigma_{t} w \in G_{1} \text { for Lebesgue a.e. } t \in \mathbf{R}\right\},
$$

then $G_{2} \in \mathcal{G}^{0}$ and $Q_{m}\left(W \backslash G_{2}\right)=0$. Clearly $G_{2}$ is $\left(\sigma_{s}\right)$-invariant. We now define

$$
\begin{aligned}
J^{1}(w) & =\int_{0}^{1} J^{\circ}\left(\sigma_{s} w\right) d s, \quad w \in G_{2} ; \\
& =0, \quad w \notin G_{2} .
\end{aligned}
$$


Then $J^{1} \circ \sigma_{s}=J^{1}$ identically in $(s, w)$ and $Q_{m_{i}}\left(J^{1} \neq J^{0}\right)=0$ so that $J^{1}$ is a $\mathcal{G}_{\alpha}^{m_{i}}$-measurable version of $d Q_{\xi_{i}} / d Q_{m_{i}}$. Finally, define

$$
J=J^{1} 1_{\left\{J^{1}<\infty\right\}} 1_{\{\alpha=-\infty\}}
$$

to obtain the required version of $d Q_{\xi_{i}} / d Q_{m}$.

The convex cone Pur is "solid" for the weak order in the sense that if $\xi \leftarrow m$ and $m \in$ Pur, then $\xi \in$ Pur. This fact is evident from (6.4). The same can be said of the cone Pot, but this is not quite evident.

(6.7) Proposition. Let $\xi \in$ Exc and $m \in$ Pot with $\xi \leftarrow m$. Then $\xi \in$ Pot. Indeed, if $\kappa$ is as in (6.3) (and $J=0$ since $m \in \operatorname{Pot} \subset$ Pur) then $\xi=\pi U$ where

$$
\pi(f)=Q_{m} \int_{[0,1]} f\left(Y_{t+}\right) \kappa(d t) .
$$

ProOF. Let $m=\gamma U \in$ Pot and $\xi \in$ Exc with $\xi \leftarrow m$ via the $\operatorname{HRM} \kappa$, as in (6.4). Again, $J=0$ since $m \in$ Pot $\subset$ Pur and consequently $\xi \in$ Pur. Thus $\xi=\int_{0}^{\infty} \mu_{t} d t$ where $\mu=\left(\mu_{t}\right)$ is an entrance law with Kuznetsov measure $Q_{\mu}$, and $Q_{\mu}=\mathbf{P}_{\kappa}$. Let $q$ and $\Omega_{q}$ be as in (2.8). We claim that $\Omega_{q}^{c}$ is $m$ - $\tau$-evanescent. Clearly $\Omega_{q}^{c}$ is $m$ - $\tau$-polar (cf. (3.6)), so that it suffices to check that $Q_{m}\left(\tau_{\alpha} \in \Omega_{q}^{c} ; \alpha \in \mathbf{R}\right)=0$. But this follows from (2.8) since $m \in$ Pot. Thus $\Omega_{q}^{c}$ is $m$ - $\tau$-evanescent and so $Q_{\mu}\left(\Omega_{q}^{c}\right)=\mathbf{P}_{\kappa}\left(\Omega_{q}^{c}\right)=0$. A second application of (2.8) reveals that $\xi \in$ Pot. Finally, let $g \in B^{+}$with $\lambda(g)=1$. Using (2.8) and (2.5) we may compute, using the notation $\xi=\pi U$,

$$
\begin{aligned}
\pi(f) & =Q_{\mu}\left(f\left(Y_{0+}\right)\right)=Q_{\xi}\left(f\left(Y_{\alpha+}\right) g(\alpha) ; \alpha \in \mathbf{R}\right) \\
& =Q_{m}\left(\int_{\mathbf{R}} f\left(Y_{\alpha+}\right) \circ b_{t} g(\alpha) \circ b_{t} \kappa(d t)\right) \\
& =Q_{m}\left(\int_{\mathbf{R}} f\left(Y_{t+}\right) g(t) \kappa(d t)\right)=\lambda(g) \mathbf{P}_{\kappa}\left(f\left(Y_{0+}\right)\right) \\
& =\mathbf{P}_{\kappa}\left(f\left(Y_{0+}\right)\right)
\end{aligned}
$$

as required.

There are two other orders on Exc that are of interest. Recall that $\xi$ is simply dominated by $m$ if $\xi(A) \leq m(A), \forall A \in \mathcal{E}$. In this case we write $\xi \leq m$. We say that $\xi$ is strongly dominated by $m$, and write $\xi \prec m$, provided there exists $\gamma \in$ Exc such that $\xi+\gamma=m$. Of course $\xi \prec m \Rightarrow \xi \leq m$, and both the simple and the strong order are true partial orders on Exc. The weak order is only a "preorder": although $\leftarrow$ is transitive, we can have $\xi \leftarrow m, m \leftarrow \xi$ and yet $\xi \neq m$. For instance, take $\xi=2 m$. We leave it to the reader to verify the transitivity of $\leftarrow$ by checking that if $\xi \leftarrow \gamma$ via $\kappa_{1}$ and $J_{1}$ and $\gamma \leftarrow m$ via $\kappa_{2}$ and $J_{2}$, then $\xi \leftarrow m$ via $\kappa$ and $J=J_{1} J_{2}$, where $\kappa$ is the $Q_{m}$-dual copredictable projection of the HRM $\left.\left.\left(\kappa_{2}\right]-\infty, s\right]+J_{2} 1_{\{\alpha=-\infty\}}\right) \kappa_{1}(d s)$.

The conditions (i) and (ii) in (6.6) characterizing the relation $\xi \leftarrow m$ may be hard to verify in specific cases. For instance, if $\xi \leq m$ then $\xi \leftarrow m$, but this is not an obvious consequence of (6.3). We shall develop several "analytic" criteria for $\xi \leftarrow m$. One of these involves viewing the representing HRM $\kappa$ as an additive functional over the dual process $\left(\hat{X}, \hat{P}^{x}\right)$. We now develop in some detail the discussion initiated after (6.2). Fix $\xi$ and $m$ in Exc with $\xi \leftarrow m$; let $\kappa$ and $J$ be as in (6.3) and assume that $\kappa$ is perfect (as we may). The definitions of $l, \Lambda, \bar{Y}$, and 
$\hat{X}$ are as in previous sections relative to $Q_{m}$. These objects, when taken relative to $Q_{\xi}$, will be denoted $l^{*}, \Lambda^{*}, \bar{Y}^{*}$, and $\hat{X}^{*}$.

Define an increasing process $\left(B_{s}: s \in[0,+\infty]\right)$ on $\hat{\Omega}$ by setting $B_{0}=0$ and

$$
B_{s}=\kappa\left[-s, 0\left[, \quad s>0 ; \quad B_{\infty}=\kappa\right]-\infty, 0\left[+J 1_{\{\alpha=-\infty\}} .\right.\right.
$$

Since $\kappa(B) \in \mathcal{G}^{*}(I)$ if $B \in B \cap I$, it is clear that $\left(B_{s}\right)$ is adapted to $\left(\hat{\mathcal{F}}_{s+}^{*}\right)$, where $\hat{\mathcal{F}}_{t}^{*}$ denotes the universal completion of $\hat{f}_{t}^{0}$. $\hat{\Omega}$,

Also, since $\kappa\left(\left[-t, 0[) \in \mathcal{G}^{*}(]-\infty, 0[), \kappa\left[-t, 0\left[\circ \hat{\tau}_{0}=\kappa[-t, 0[\right.\right.\right.\right.$. Consequently, on

$$
\begin{aligned}
B_{t+s} & =\kappa[-(t+s), 0[=\kappa[-s, 0[+\kappa[-(t+s),-s[ \\
& =B_{s}+\kappa\left[-t, 0\left[\circ \sigma_{-s}=B_{s}+\kappa\left[-t, 0\left[\circ k_{0} \circ \sigma_{-s}\right.\right.\right.\right. \\
& =B_{s}+B_{t} \circ \hat{\theta}_{s} .
\end{aligned}
$$

That is, $\left(B_{s}\right)$ is an AF of $\hat{X}$. Set

$$
\hat{u}(x)=\hat{P}^{x}\left(B_{\infty}\right)=\hat{P}^{x}(\kappa]-\infty, 0\left[+J 1_{\{\alpha=-\infty\}}\right) .
$$

If $f \in \mathcal{E}^{+}$with $f>0$ and $\xi(f)<\infty$, then by the computation following (6.2), $\xi(f)=m(\hat{u} \cdot f)<\infty$ and so $m\{\ddot{u}=+\infty\}=0$. Also

$$
\begin{aligned}
\hat{P}_{s} \hat{u}(x) & =\hat{P}^{x}\left(B_{\infty} \circ \hat{\theta}_{s}\right)=\hat{P}^{x}(\kappa]-\infty,-s\left[+J 1_{\{\alpha=-\infty\}}\right), \\
\uparrow & \hat{P}^{x}\left(B_{\infty}\right)=\hat{u}(x), \quad \text { as } s \downarrow 0 .
\end{aligned}
$$

That is, $\hat{u}$ is excessive for the semigroup $\left(\hat{P}_{s}\right)$. By Lemma 2 of [9], the process $t \rightarrow \hat{u}\left(\hat{X}_{t}\right)$ has left and right limits on $\left[0,+\infty\left[\right.\right.$, a.s. $\hat{P}^{x}$ for each $x \in E$. Thus we may define a rc process $H=\left(H_{t}\right)$ by

$$
H_{t}=\lim _{s \downarrow t} \hat{u}\left(\hat{X}_{s}\right), \quad t \geq 0 .
$$

Note that $H$ is a supermartingale under $\hat{P}^{x}$ provided $\hat{u}(x)=\hat{P}^{x}\left(H_{0}\right)<\infty$. In this case $\left(B_{s}\right)$ is rc and is the integrable increasing process in the Doob-Meyer decomposition of $H$.

Fix now $x \in\{\hat{u}<\infty\}$ so that $B_{s}<\infty, \forall s \geq 0$ a.s. $\hat{P}^{x}$. Recalling the proof of (5.27), the discrete part of $1_{\Lambda} * \kappa$ is $Q_{m}$-indistinguishable from $\sum_{s \in \mathbf{R}} j\left(\bar{Y}_{s}\right) \varepsilon_{s}$ where $\{j>0\}$ is a Borel $m$-semipolar set. It follows that

$$
\Delta B_{s}=B_{s}-B_{s-}=j\left(\hat{X}_{s}\right), \quad \forall s \in \hat{\Lambda} \text {, a.s. } \hat{P}^{x} .
$$

Let $T$ be a predictable time of $\left(\hat{\mathcal{F}}_{t+}^{*}\right)$. By the moderate Markov property (4.4),

$$
\begin{gathered}
\hat{P}_{x}\left(B_{\infty}-B_{T} \mid \hat{\mathcal{F}}_{T-}^{*}\right)=\hat{P}^{x}\left(B_{\infty} \circ \hat{\theta}_{T} \mid \hat{\mathcal{F}}_{T-}^{*}\right) \\
=\hat{u}\left(\hat{X}_{T}\right), \quad \text { a.s. } \hat{P}^{x} \text { on }\{0<T<\infty\} .
\end{gathered}
$$

If $S$ is an $\left(\hat{\mathcal{F}}_{t+}^{*}\right)$-optional time, then $S+t$ is predictable for each $t>0$. Taking $T=S+t$ in (6.11) and letting $t \downarrow 0$ we obtain

$$
\hat{P}^{x}\left(B_{\infty}-B_{S} \mid \hat{\mathcal{F}}_{S+}^{*}\right)=H_{S}, \quad \text { a.s. } \hat{P}^{x} \text { on }\{S<\infty\} .
$$

Now let $T$ be an $\left(\hat{\mathcal{F}}_{t+}^{*}\right)$-predictable time and let $\left(S_{n}\right)$ be a sequence of $\left(\hat{\mathcal{F}}_{t+}^{*}\right)$-optional times announcing $T$. Taking $S=S_{n}$ in (6.12) and letting $n \uparrow \infty$,

$$
\hat{P}^{x}\left(B_{\infty}-B_{T-} \mid \hat{\mathcal{F}}_{T-}^{*}\right)=H_{T-}, \quad \text { a.s. } \hat{P}^{x} \text { on }\{0<T<\infty\} \text {. }
$$


But on $\{T \in \hat{\Lambda}\}$ we have $B_{\infty}-B_{T-}=B_{\infty}-B_{T}+j\left(\hat{X}_{T}\right)$, a.s. $\hat{P}^{x}$. Comparing (6.13) and (6.11) we conclude that

$$
\hat{u}\left(\hat{X}_{T}\right)+j\left(\hat{X}_{T}\right)=H_{T-}, \quad \text { a.s. } \hat{P}^{x} \text { on }\{T \in \hat{\Lambda}\} .
$$

Thus, if we define $\bar{u}=\hat{u}+j$, then by the section theorem the processes $1_{\hat{\Lambda}}(t) \bar{u}\left(\hat{X}_{t}\right)$ and $1_{\hat{\Lambda}}(t) H_{t-}$ are $\hat{P}^{x}$-indistinguishable. In particuiar, $t \rightarrow \bar{u}\left(\hat{X}_{t}\right)$ is lc on $\hat{\Lambda}$, a.s., $\hat{P}^{x}$. Finally, note that

$$
\lim _{s \downarrow t} \bar{u}\left(\hat{X}_{t}\right)=H_{t}, \quad \forall t \geq 0, \text { a.s., } \hat{P}^{x} .
$$

Of course, all of these results hold for any $x \in\{\hat{u}<\infty\}$.

(6.16) Proposition. Let $T$ be a copredictable time (that is, $\rrbracket-\infty, T \rrbracket \in \hat{P}^{0}$ ). Then for any $f \in \mathcal{E}^{+}$,

$$
Q_{\xi}\left(f \circ \bar{Y}_{T} ; T \in \Lambda\right)=Q_{m}\left(f \circ \bar{Y}_{T} \bar{u} \circ \bar{Y}_{T} ; T \in \Lambda\right) .
$$

Proof. We can assume that $T=-\infty$ if $T<\alpha$, replacing $T$ by $T^{\prime}=T$ on $\{T \geq \alpha\},=-\infty$ on $\{T<\alpha\}$ if necessary. We then have (cf. [2, p. $462 \mathrm{ff}]$.

$$
\begin{array}{rlrl}
T \circ b_{s} & =T, & & \text { if } T \geq s, \\
& =-\infty, & \text { if } T<s,
\end{array}
$$

for any $s \in \mathbf{R}$. Thus $b_{s}^{-1}\{T \in \Lambda\}=b_{s}^{-1}\left\{l_{T}>0\right\}=\left\{l_{T}>0 ; T \geq s\right\}$. Using (6.4) we compute

$$
\begin{aligned}
Q_{\xi}\left(f \circ \bar{Y}_{T} ; T \in \Lambda\right)= & Q_{m}\left(\int_{\mathbf{R}} f\left(\bar{Y}_{T}\right) \circ b_{s} 1_{\{T \in \Lambda\}} \circ b_{s} \kappa(d s)\right) \\
& +Q_{m}\left(f \circ \bar{Y}_{T} J ; T \in \Lambda ; \alpha=-\infty\right) \\
= & \left.\left.Q_{m}\left(f \circ \bar{Y}_{T}\{\kappa]-\infty, T\right]+J 1_{\{\alpha=-\infty\}}\right\} ; T \in \Lambda\right) \\
= & Q_{m}\left(f \circ \bar{Y}_{T}\left\{B_{\infty} \circ \tau_{T}+j\left(\bar{Y}_{T}\right)\right\} ; T \in \Lambda\right) \\
= & Q_{m}\left(f \circ \bar{Y}_{T} \bar{u}\left(\bar{Y}_{T}\right) ; T \in \Lambda\right) .
\end{aligned}
$$

(6.18) REMARKS. (a) We emphasize that it is $\bar{Y}_{T}$ and $\Lambda$ on the left side of (6.17), not $\bar{Y}_{T}^{*}$ and $\Lambda^{*}$. The relationship between these objects will be clarified shortly.

(b) It follows from (6.17) that the Borel set $\{\bar{u}=+\infty\}$ is $m$-polar; thus $\{\hat{u}=$ $+\infty\}$ is also $m$-polar. It is easy to check that $\bar{u}$ is a version of $d \xi / d m$ having the properties listed in (4.15). Finally, note that $\hat{u}$ is the "excessive regularization" of $\bar{u}$ relative to $\left(\hat{P}_{s}\right)$. Indeed, since $\kappa$ is carried by $\llbracket \alpha, \beta \llbracket, B_{\infty}=0$ on $\{\hat{\xi}=0\}$. Thus

$$
\begin{aligned}
\hat{P}_{s} \bar{u}(x) & =\hat{P}^{x}\left(H_{s-} ; s \in \hat{\Lambda}\right) \uparrow \hat{P}^{x}\left(H_{0} ; \hat{\xi}>0\right), \quad \text { as } s \downarrow 0 \\
& =\hat{P}^{x}\left(B_{\infty} ; \hat{\xi}>0\right)=\hat{P}^{x}\left(B_{\infty}\right)=\hat{u}(x) .
\end{aligned}
$$

We continue to assume that $\xi \leftarrow m$, and we follow the previously established notation. Define a family of measures $\left(\hat{P}_{*}^{x}, x \in E_{\Delta}\right)$ on $\left(\hat{\Omega}, \hat{\mathcal{F}}^{0}\right)$ by

$$
\hat{P}_{*}^{x}(F)=\left\{\hat{P}^{x}\left(\int_{[0,+\infty]} F \circ b_{-s} d B_{s}\right)+F([\Delta]) j(x)\right\} / \bar{u}(x),
$$

where the ratio is taken to be 0 unless $0<\bar{u}(x)<\infty$. Note that $x \rightarrow \hat{P}_{*}^{x}(F)$ is only universally measurable on $E$. One can modify $\hat{P}_{*}^{x}$ on an $m$-polar set of $x$ 's so as 
to obtain a Borel kernel, but we prefer to have the explicit formula (6.19). We are therefore making a slight abuse of the term "moderate Markov dual", defined in (4.1), in the following result. This result is a variation on Nagasawa's time reversal theorem. See $[\mathbf{2 6}, \mathbf{2 9}, \mathbf{4 0}]$ for versions of this result under duality hypotheses. See also [15] for a general result in this vein.

(6.20) THEOREM. With $\xi \leftarrow m$ as above, let $\hat{P}_{*}^{x}$ be defined as in (6.19). Then $\left(\hat{P}_{*}^{x}\right)$ is a moderate Markov dual for $\left(\hat{P}^{x}\right)$, relative to $\xi$. That is, (4.2) and (4.4) hold with $Q_{m}, \hat{P}^{x}, \hat{X}$, and $\bar{Y}$ replaced by $Q_{\xi}, \hat{P}_{*}^{x}, \hat{X}^{*}$, and $\bar{Y}^{*}$. In particular, $\Lambda^{*} \subset \Lambda$ modulo $I^{\xi}$, and $t \rightarrow \hat{u}\left(\bar{Y}_{t}\right) / \bar{u}\left(\bar{Y}_{t}\right)(0 / 0=\infty / \infty=0)$ is a version of $l^{*}$.

ProOF. Let $T$ be a copredictable time. Using (6.4) and (6.17),

$$
\begin{aligned}
Q_{\xi}\left(l_{T}^{*} ;-\infty<T<\beta\right) & =Q_{\xi}(\alpha<T<\beta) \\
& =Q_{m}(\kappa]-\infty, T[T<\beta)+Q_{m}\left(J 1_{\{\alpha=-\infty\}} ;-\infty<T<\beta\right) \\
& =Q_{m}\left(B_{\infty} \circ \tau_{T} ;-\infty<T<\beta\right)=Q_{m}\left(\hat{u}\left(\bar{Y}_{T}\right) ; T \in \Lambda\right) \\
& =Q_{m}\left((\hat{u} / \bar{u})\left(\bar{Y}_{T}\right) ; \bar{u}\left(\bar{Y}_{T}\right) ; T \in \Lambda\right) \\
& =Q_{\xi}\left((\hat{u} / \bar{u})\left(\bar{Y}_{T}\right) ;-\infty<T<\beta\right) .
\end{aligned}
$$

It follows that $(\hat{u} / \bar{u})\left(\bar{Y}_{t}\right)$ is a version of $l_{t}^{*}$. In particular, since $\bar{Y}_{t}=\Delta$ if $t \notin \Lambda$, we must have $\Lambda^{*} \subset \Lambda$ modulo $I^{\xi}$. Clearly $\bar{Y}=\bar{Y}^{*}$ on $\Lambda \cap \Lambda^{*}=\Lambda^{*}$. Thus, $l^{*},(\hat{u} / \bar{u})\left(\bar{Y}_{.}^{*}\right)$, and $(\hat{u} / \bar{u})(\bar{Y}$.$) are Q_{\xi^{-}}$indistinguishable each from the others. A computation similar to the above with $l_{T}^{*}$ replaced by $F \circ \hat{\tau}_{T} l_{T}^{*}\left(F \in\left(\hat{\mathcal{F}}^{0}\right)^{+}\right)$, shows that

$$
\begin{aligned}
Q_{\xi}\left(F \circ \hat{\tau}_{T} ; T \in \Lambda^{*}\right) & =Q_{m}\left(\hat{P}_{*}^{\bar{Y}_{T}}(F) \bar{u}\left(\bar{Y}_{T}\right) 1_{E}\left(\bar{Y}_{T}^{*}\right) ; T \in \Lambda\right) \\
& =Q_{\xi}\left(\hat{P}_{*}^{\bar{Y}^{*}}(F) ; T \in \Lambda^{*}\right) .
\end{aligned}
$$

Thus the version of (4.2) appropriate to $Q_{\xi}$ is valid. The verification of (4.4) for $\hat{P}_{*}^{x}$ now follows from the explicit formula (6.19). See for example Azema [2, p. 484] for a very similar computation.

(6.22) REMARK. Let $\left(\hat{P}_{s}^{*}\right)$ denote the semigroup associated with $\left(\hat{P}_{*}^{x}\right)$. One checks that

$$
\begin{aligned}
\hat{P}_{s}^{*} f(x) & =\hat{P}_{s}(\bar{u} f)(x) / \bar{u}(x), \quad 0<\bar{u}(x)<\infty, \\
& =0, \quad \text { otherwise, }
\end{aligned}
$$

so that $\left(\hat{P}_{s}^{*}\right)$ is the $\bar{u}$-transform of $\left(\hat{P}_{s}\right)$.

We will now set down our first "analytic" criterion for $\xi \leftarrow m$. Actually, we will only prove the necessity of this condition in the result to follow. The sufficiency will follow easily when we consider a second analytic criterion. Recall the balayage operations $L_{T}$ and $L_{B}$ defined in $\S 5$ of [19]. Let $T: W \rightarrow \mathbf{R} \cup\{-\infty,+\infty\}$ be a stopping time of $\left.\left.\left(\mathcal{G}_{t+}^{*}\right) \equiv\left(\bigcap_{s>t} \mathcal{G}^{*}(]-\infty, s\right]\right)\right)$ such that

$$
\begin{aligned}
& \text { (i) } \alpha \leq T<\beta \quad \text { on }\{T<+\infty\} \text {; } \\
& \text { (ii) } T=s+T \circ \sigma_{s}, \quad \forall s \in \mathbf{R} .
\end{aligned}
$$

Such a time is called an intrinsic time in [19]. For example, if $A$ is a Borel set in $E$ then

$$
T_{A}=\inf \left\{t>\alpha: Y_{t} \in A\right\}
$$


is an intrinsic time. Given an intrinsic time $T$, the balayage $L_{T}:$ Exc $\rightarrow$ Exc is defined by

$$
L_{T} m(f)=Q_{m}\left(f \circ Y_{t} ; T<t\right), \quad f \in \mathcal{E}^{+} .
$$

One checks that $L_{T} m \in \mathrm{Exc}, L_{T} m$ is independent of $t \in \mathbf{R}, L_{T} m \leq m$ and

$$
Q_{L_{T} m}(F)=Q_{m}\left(F \circ b_{T} ; T<+\infty\right), \quad F \in\left(\mathcal{G}^{0}\right)^{+} .
$$

In case $T=T_{A}$ we write $L_{A}$ for $L_{T_{A}}$. By the stationarity of $Q_{m}$ we must have $Q_{m}(T=t)=0$ for any $t \in \mathbf{R}$; thus the condition $\{T<t\}$ in (6.25) may be replaced by $\{T \leq t\}$. From this fact it follows easily that $L_{A} m=m$ on $A$. See [19] for all of these assertions.

(6.27) Proposition. Let $\xi$ and $m$ be excessive measures with $\xi \leftarrow m$. Then $\xi<<m$ and if $\phi$ is the version of $d \xi / d m$ provided by (4.15), then

$$
L_{\{\phi>t\}} \xi \downarrow 0 \text { as } t \uparrow \infty .
$$

ProOF. It is clear from the discussion following (6.2) that $\xi<<m$. Let $\phi=$ $d \xi / d m$ as provided by (4.15) so that $t \rightarrow \phi\left(\bar{Y}_{t}\right)$ is rc on $\rrbracket \alpha,+\infty \llbracket$, a.e., $Q_{m}$. Let $\bar{u}=\hat{u}+j$ be as in the discussion following (6.14). Then $t \rightarrow \bar{u}\left(\hat{X}_{t}\right)$ is lc on $\rrbracket 0, \hat{\zeta} \llbracket$ a.s. $\hat{P}^{x}$ whenever $\bar{u}(x)<\infty$. It follows that $t \rightarrow \bar{u}\left(\bar{Y}_{t}\right)$ is rc on $\rrbracket \alpha,+\infty \llbracket$ a.e. $Q_{m}$. Since $\phi$ and $\bar{u}$ are both versions of $d \xi / d m$ (cf. (6.17)) we have $Q_{m}\left(\phi\left(\bar{Y}_{t}\right) \neq \bar{u}\left(\bar{Y}_{t}\right)\right)=0$ for each $t \in \mathbf{R}$. By right continuity, $\phi\left(Y_{t}\right)$ and $\bar{u}\left(Y_{t}\right)$ are $Q_{m}$-indistinguishable on $\rrbracket \alpha,+\infty \llbracket$. It now follows from (6.17) that $\phi\left(Y_{t}\right)$ and $\bar{u}\left(Y_{t}\right)$ are $Q_{\xi}$-indistinguishable on $\rrbracket \alpha,+\infty \llbracket$. Thus, $T_{\{\phi>t\}}=T_{\{\bar{u}>t\}}$ a.e. $Q_{\xi}$ for any $t>0$, and so

$$
L_{\{\phi>t\}} \xi=L_{\{\bar{u}>t\}} \xi, \quad \forall t>0 .
$$

Therefore it suffices to show that $L_{\{\bar{u}>t\}} \xi \downarrow 0$ as $t \uparrow \infty$. Now recall the discussion following (6.10); in particular recall the rc supermartingale $\left(H_{s}\right)$ and the relationships

$$
\begin{aligned}
& H_{t}=\lim _{s \downarrow t} \bar{u}\left(\hat{X}_{s}\right), \quad \forall t \geq 0, \text { a.s. } \hat{P}^{x}, \\
& \left.\bar{u}\left(\hat{X}_{t}\right)=H_{t-}, \quad \forall t \in\right] 0, \hat{\zeta}\left[, \text { a.s. } \hat{P}^{x},\right.
\end{aligned}
$$

whenever $\bar{u}(x)<\infty$. Thus, since both $\left(H_{s}\right)$ and $\bar{u}\left(\hat{X}_{s}\right)$ vanish on $\rrbracket \hat{\zeta},+\infty \llbracket$, if we set

$$
S(t)=\inf \left(s>0: H_{s}>t\right), \quad t>0,
$$

then $S(t)=\inf \left(s>0: \bar{u}\left(\hat{X}_{s}\right)>t\right)$ a.s. $\hat{P}^{x}$, if $\hat{u}(x)<\infty$. Now since $\left(H_{s}\right)$ is a rc supermartingale almost every path of $\left(H_{s}\right)$ is bounded. Thus, $S(t)=+\infty$ for all large $t$, a.s. $\hat{P}^{x}$. In particular, if $\bar{u}(x)<\infty$, then

$$
\hat{P}^{x}\left(B_{\infty}-B_{S(t)}\right) \downarrow 0 \quad \text { as } t \uparrow \infty .
$$

Recalling the definition (6.24) of $T_{A}$ for $A \in \mathcal{E}$, note that on $\{\alpha<0\}$,

$$
\begin{aligned}
b_{s}^{-1}\left\{T_{A}<0\right\} & =\left\{\bar{Y}_{u} \in A \text { for some } u \in\right] s, 0[\} \\
& =\left\{\hat{X}_{u} \in A \text { for some } u \in\right] 0,-s[\} .
\end{aligned}
$$


Thus, if $f \in \mathcal{E}^{+}$with $\xi(f)<\infty$,

$$
\begin{aligned}
L_{\{\bar{u}>t\}} \xi(f)= & Q_{\xi}\left(f \circ Y_{0} ; T_{\{\bar{u}>t\}}<0\right) \\
= & Q_{m}\left(\int_{\mathbf{R}} f \circ Y_{0} \circ b_{s} 1_{\left\{T_{\{\bar{u}>t\}<0\}} \circ b_{s} \kappa(d s)\right)}\right. \\
& +Q_{m}\left(f \circ Y_{0} J ; T_{\{\bar{u}>t\}}<0\right) \\
= & Q_{m}\left(f \circ Y_{0}\left(\int_{j 0,+\infty]} 1_{\{S(t)<u\}} d B_{u}\right) \circ \hat{\tau}_{0}\right) \\
= & Q_{m}\left(f \circ Y_{0}\left(B_{\infty}-B_{S(t)}\right) \circ \hat{\tau}_{0}\right) \\
= & Q_{m}\left(f \circ Y_{0} \hat{P}^{Y_{0}}\left(B_{\infty}-B_{S(t)}\right)\right) .
\end{aligned}
$$

Combining (6.29) and (6.30) we see that if $\xi(f)<\infty$, then $L_{\{\bar{u}>t\}} \xi(f) \downarrow 0$, as required.

Our second criterion for $\xi \leftarrow m$ involves the "reduite" operation on excessive measures, defined as follows. Let $\pi$ be a measure on $E$ dominated by some element of Exc. The reduite of $\pi, R \pi$ is then defined as the greatest lower bound (in the lattice Exc with the simple order) of the set $\{\gamma \in$ Exc: $\gamma \geq \pi\}$. In particular, if $\xi \in$ Exc and $A \in \mathcal{E}$ we write $R_{A} \xi$ for $R\left(1_{A} \cdot \xi\right)$. It is shown in [18] that if $\xi \in \operatorname{Exc}$ then $R_{A} \xi=L_{\Pi(A)} \xi$, where $\Pi(A)$ is the Lebesgue penetration time of $A$. In particular, $R_{A}$ is additive on Exc.

In what follows, if $\xi$ and $\eta$ are $\sigma$-finite measures on $E$, then statements of the type $\xi \leq \eta$ on $A(A \in \mathcal{E})$ should be interpreted as follows. Let $\lambda=\xi+\eta$ so that $\xi<<\lambda, \eta<<\lambda$ and the densities $d \xi / d \lambda$ and $d \eta / d \lambda$ can be taken to be bounded. Then $\xi \leq \eta$ on $A$ means $d \xi / d \lambda \leq d \eta / d \lambda$ a.e. $\lambda$ on $A$.

We require several results regarding the reduite operation. These results are all due originally to Mokobodski; a good reference is [8]. See also Vol. 3 of [12]. The proof of (6.31) is taken from Heath [42], while the proof of (6.33) seems to be new.

(6.31) Lemma. Suppose that $\rho=R \pi$ and let $A \supset\{\varepsilon \rho \leq \pi\}$ where $0<\varepsilon<1$. Then $R_{A} \rho=\rho$.

ProOF. Write $\gamma=R_{A} \rho$ so that $\gamma \leq \rho$. Then on $E \backslash A$ we have $\pi \leq \varepsilon \rho \leq$ $\varepsilon \rho+(1-\varepsilon) \gamma$, since $\gamma \geq 0$. On $A$ we have $\pi \leq \rho=\varepsilon \rho+(1-\varepsilon) \gamma$, since $\rho=\gamma$ on $A$. Thus, $\pi \leq \varepsilon \rho+(1-\varepsilon) \gamma$ and since $R$ is evidently monotone and since $R \xi=\xi$ if $\xi \in \mathrm{Exc}$,

$$
\rho=R \pi \leq R(\varepsilon \rho+(1-\varepsilon) \gamma) \leq \varepsilon \rho+(1-\varepsilon) \gamma .
$$

Rearranging (6.32) we obtain $\rho \leq \gamma$, and so $\rho=\gamma$ as required.

(6.33) COROLlaRY. Let $\xi, m_{1}$, and $m_{2}$ be excessive measures with $\xi \leq m_{1}+$ $m_{2}$. Then there exists excessive measures $\xi_{1} \leq m_{1}$ and $\xi_{2} \leq m_{2}$ such that $\xi_{1}+\xi_{2}=$ $\xi$.

PROOF. Define $\xi_{2}=R\left(\xi-m_{1}\right)$, that is, $\xi_{2}=\inf \left\{\gamma \in\right.$ Exc: $\left.\gamma+m_{1} \geq \xi\right\}$. Similarly, set $\xi_{1}=R\left(\xi-\xi_{2}\right)$. Clearly $\xi_{1} \leq m_{1}, \xi_{2} \leq m_{2}$, and $\xi_{1}+\xi_{2} \geq \xi$. Given $\varepsilon \in] 0,1\left[\right.$ let $A=\left\{\varepsilon\left(\xi_{1}+\xi_{2}\right) \leq \xi\right\}$. Then

$$
A_{1} \equiv\left\{\varepsilon \xi_{1}+\xi_{2} \leq \xi\right\} \subset A
$$


and so, by (6.31), $\xi_{1}=R_{A_{1}} \xi_{1} \leq R_{A} \xi_{1} \leq \xi_{1}$. That is, $\xi_{1}=R_{A} \xi_{1}$. Similarly, if we set $A_{2}=\left\{\varepsilon \xi_{2}+m_{1} \leq \xi\right\}$, then since $m_{1} \geq \xi_{1} \geq \varepsilon \xi_{1}$, we have $A_{2} \subset A$ and so $\xi_{2}=R_{A_{2}} \xi_{2} \leq R_{A} \xi_{2} \leq \xi_{2}$. Thus $\xi_{2}=R_{A} \xi_{2}$. Finally,

$$
\xi_{1}+\xi_{2}=R_{A}\left(\xi_{1}+\xi_{2}\right)=\varepsilon^{-1} R_{A}\left(\varepsilon\left(\xi_{1}+\xi_{2}\right)\right) \leq \varepsilon^{-1} R_{A} \xi \leq \varepsilon^{-1} \xi
$$

where the inequality follows since $\varepsilon\left(\xi_{1}+\xi_{2}\right) \leq \xi$ on $A$. Thus $\xi_{1}+\xi_{2} \leq \varepsilon^{-1} \xi$ and letting $\varepsilon \uparrow 1$ we obtain $\xi_{1}+\xi_{2} \leq \xi$.

(6.34) COROllary. Given $\xi$ and $m$ in Exc set $\rho=R(\xi-m)$. Then $\rho \prec \xi$ so that there exists $\gamma \in \operatorname{Exc}$ with $\rho+\gamma=\xi$. One then has $\gamma \leq m$ and, indeed, $\gamma=\sup \{\eta \in \operatorname{Exc}: \eta \leq m, \eta \prec \xi\}$.

ProOF. Clearly $\xi \leq \rho+m$. By (6.33) there are excessive measures $\hat{\rho} \leq \rho, \hat{m} \leq m$ such that $\xi=\hat{\rho}+\hat{m}$. But then $\hat{\rho}+m \geq \hat{\rho}+\hat{m}=\xi$, so $\hat{\rho} \geq \rho(=R(\xi-m))$. Thus $\rho=\hat{\rho}$. Set $\gamma=\hat{m}$ so that $\rho+\gamma=\xi, \gamma \prec \xi, \gamma \leq m$. If $\tilde{\gamma} \in \operatorname{Exc}$ with $\tilde{\gamma} \prec \xi$ and $\tilde{\gamma} \leq m$, then there exists $\tilde{\rho} \in \operatorname{Exc}$ with $\tilde{\gamma}+\tilde{\rho}=\xi$. But then $\tilde{\rho}+m \geq \tilde{\rho}+\tilde{\gamma}=\xi$, so $\tilde{\rho} \geq \rho$. Since $\tilde{\gamma}+\tilde{\rho}=\xi$ we must have $\tilde{\gamma} \leq \gamma$. That is, $\gamma=\sup \{\eta \in \operatorname{Exc}: \eta \prec \xi, \eta \leq m\}$.

The following is an adaptation of a result that Heath [42] ascribes to Mokobodski. For a proof (modeled on [42]) see [18].

(6.35) Proposition. Fix $\xi$ and $m$ in Exc. For each $t \geq 0$ let $\gamma_{t}=R(\xi-t \cdot m)$ so that $\xi=\gamma_{t}+\rho_{t}$ where $\rho_{t} \in \operatorname{Exc}$ with $\rho_{t} \leq t \cdot m$ (see (6.34)). Then

(i) For each $f \in \mathcal{E}^{+}$with $\xi(f)<\infty, t \rightarrow \gamma_{t}(f)$ is decreasing and convex on $[0,+\infty[$

(ii) The right hand derivatives $\delta_{t}(f)=-d^{+} \gamma_{t}(f) / d t^{+}$constitute a family $\left\{\delta_{t}\right.$ : $t \geq 0\}$ of excessive measures. Moreover, there exists an increasing family $\{T(t): t \geq$ $0\}$ of $\left(\mathcal{G}_{t}^{*}\right)$-optional, intrinsic times such that $\delta_{t}=L_{T(t)} m, \forall t \geq 0$.

(iii) Set $\gamma_{\infty}=\lim _{t \rightarrow+\infty} \downarrow \gamma_{t}=\inf \left\{\gamma_{t}: t \geq 0\right\}$. Then

$$
\xi=\gamma_{\infty}+\int_{0}^{\infty} L_{T(t)} m d t
$$

Here now is the main result of this section, characterizing in analytic terms the weak order.

(6.37) THEOREM. Let $\xi$ and $m$ be excessive measures. Then the following are equivalent.

(i) $\xi \leftarrow m$.

(ii) $\xi<<m$ and $L_{\{\phi>t\}} \xi \downarrow 0$ as $t \uparrow+\infty$, where $\phi=d \xi / d m$ is as in (4.15).

(iii) $\lim _{t \uparrow \infty} \gamma_{t} \equiv \lim _{t \uparrow \infty} R(\xi-t \cdot m)=0$.

PROOF. The implication (i) $\Rightarrow$ (ii) is the content of $(6.27)$. We will show that (ii) $\Rightarrow$ (iii) and (iii) $\Rightarrow$ (i).

(a) (ii) $\Rightarrow$ (iii): Let $\phi \geq 0$ be any $\mathcal{E}$-measurable version of $d \xi / d m$. We claim that $L_{\{\phi>t\}} \xi \geq \gamma_{t}$, where $\gamma_{t}=R(\xi-t \cdot m)$. Indeed, recall that $L_{\{\phi>t\}} \xi=\xi$ on $\{\phi>t\}$. Thus

$$
\xi=1_{\{\phi>t\}} \cdot \xi+1_{\{\phi \leq t\}} \xi \leq L_{\{\phi>t\}} \xi+t \cdot m .
$$

Consequently $R(\xi-t m) \leq L_{\{\phi>t\}} \xi$ as claimed. Since $L_{\{\phi>t\}} \xi \downarrow 0$ as $t \uparrow \infty$ when $\phi$ is the version of $d \xi / d m$ given in (4.15), we must have $\gamma_{t} \downarrow 0$ as well. 
(b) (iii) $\Rightarrow$ (i): By (iii) and (6.36),

$$
\xi=\int_{0}^{\infty} L_{T(t)} m d t
$$

where each $T(t)$ is an intrinsic $\left(\mathcal{G}_{t+}^{*}\right)$-optional time. Define a $\mathrm{RM} \kappa_{0}$ by

$$
\kappa_{0}(d t)=\int_{0}^{\infty} \varepsilon_{T(u)}(d t) 1_{\{T(u) \in \mathbf{R}\}} d u .
$$

One can use (5.24) to check that $\kappa_{0}$ is optional; $\kappa_{0}$ is homogeneous since $t+T(u) \circ$ $\sigma_{t}=T(u)$ for each $t \in \mathbf{R}, u \geq 0$. Moreover $\kappa_{0} \in \sigma I\left(\hat{p}^{m}\right)$, since the Palm measure of $\kappa_{0}$ is given by

$$
\begin{aligned}
\int_{0}^{\infty} & Q_{m}\left(F \circ \tau_{T(u)} ; 0<T(u) \leq 1\right) d u \\
& =\int_{0}^{\infty} Q_{m}\left(F \circ \tau_{\alpha} \circ b_{T(u)} ; 0<\alpha \circ b_{T(u)} \leq 1 ; T(u) \in \mathbf{R}\right) d u \\
& =\int_{0}^{\infty} Q_{L_{T(u)}}\left(F \circ \tau_{\alpha} ; 0<\alpha \leq 1\right) d u \\
& =Q_{\xi}\left(F \circ \tau_{\alpha} ; 0<\alpha \leq 1\right)=Q_{\mu}(F),
\end{aligned}
$$

where $\mu=\left(\mu_{t}\right)$ is the entrance law such that $\int_{0}^{\infty} \mu_{t} d t=\xi_{p}$. Next, define

$$
J=\int_{0}^{\infty} 1_{\{T(u)=-\infty\}} d u=\sup \{u: T(u)=-\infty\},
$$

where $\sup \phi=0$. Clearly $J \in\left(\mathcal{G}_{-\infty}^{m}\right)^{+} \subset\left(\mathcal{G}_{\alpha}^{m}\right)^{+}$and $J \circ \sigma_{s}=J, \forall s \in \mathbf{R}$. Moreover, if $F \in\left(\mathcal{G}^{0}\right)^{+}$,

$$
Q_{m}(F \cdot J ; \alpha=-\infty)=\int_{0}^{\infty} Q_{m}(F ; \alpha=T(u)=-\infty) d u .
$$

But using (6.38),

$$
\begin{aligned}
Q_{\xi_{i}}(F) & =\int_{0}^{\infty} Q_{m}\left(F \circ b_{T(u)} ; \alpha \circ b_{T(u)}=-\infty, T(u)<+\infty\right) d u \\
& =\int_{0}^{\infty} Q_{m}(F ; \alpha=T(u)=-\infty) d u,
\end{aligned}
$$

since $\alpha \circ b_{T(u)}=\alpha \vee T(u)$. Comparing (6.39) and (6.40) we see that

$$
Q_{\xi_{i}}(F)=Q_{m}(F J ; \alpha=-\infty), \quad F \in\left(\mathcal{G}^{0}\right)^{+} \text {. }
$$

Finally, setting $\kappa=\left(\kappa_{0}\right)^{\hat{p}}$, we see that (6.4) holds so that $\xi \leftarrow m$ as required.

(6.41) COROLlary. Let $\xi$ and $m$ be excessive measures. Then $\xi \leftarrow m$ if and only if $\xi$ can be decomposed as

$$
\xi=\sum_{n=1}^{\infty} \xi_{n},
$$

where $\xi_{n} \in \operatorname{Exc}, \xi_{n} \leq m$ for each $n \in \mathbf{N}$. In particular, if $\xi \leq t \cdot m$ for some $t \geq 0$, then $\xi \leftarrow m$.

PROOF. The necessity of (6.42) follows from (6.37) upon taking

$$
\xi_{n}=\int_{n-1}^{n} L_{T(u)} m d u, \quad n \geq 1,
$$


where $\xi=\int_{0}^{\infty} L_{T(u)} m d u$ as in (6.38). Conversely, if $\xi=\sum_{n} \xi_{n}$ where $\xi_{n} \in$ Exc with $\xi_{n} \leq m$, then

$$
\gamma_{n}=R(\xi-n \cdot m) \leq \sum_{k=n+1}^{\infty} \xi_{k} \downarrow 0 \quad \text { as } n \uparrow \infty .
$$

Since $\gamma_{t}$ decreases in $t$, we must have $\gamma_{t} \downarrow 0$ as $t \uparrow \infty$. Thus $\xi \leftarrow m$ by (6.37). The last assertion is now obvious.

(6.43) REMARK. In view of (6.41), Proposition (6.7) is a generalization of the well-known fact that if $\xi \in$ Exc, $m \in$ Pot and $\xi \leq m$, then $\xi \in$ Pot.

We close this section with an application of (6.37). This result is a "dominated decomposition" theorem extending Corollary (6.33) and is an analogue of a wellknown result of Strassen [41]. See Volume III of [12] for a discussion of Strassen's theorem. The decomposition (6.33) is quite useful as its pivotal role in [8] attests. An obvious induction followed by a limiting argument yields a useful extension of (6.33): for $\xi \in \mathrm{Exc}$

$$
\begin{aligned}
& \text { if } \xi \leq \sum_{k=1}^{\infty} m_{k} \in \text { Exc, where } m_{k} \in \text { Exc, } \forall k \in \mathbf{N}, \\
& \text { then } \xi=\sum_{k=1}^{\infty} \xi_{k}, \text { where } \xi_{k} \in \text { Exc, } \xi_{k} \leq m_{k}, \forall k \in \mathbf{N} .
\end{aligned}
$$

Our generalization (6.45) extends (6.44) in two ways. The condition $\xi \leq \sum m_{k}$ is replaced by $\xi \leftarrow \sum m_{k}$ and, secondly, the discrete parameter $k$ is allowed to be "continuous".

(6.45) THEOREM. Let $(D, D, \pi)$ be a complete $\sigma$-finite measure space and let $\left(m_{x}: x \in D\right)$ be a family of excessive measures such that $x \rightarrow m_{x}(f)$ is D-measurable for each $f \in \mathcal{E}^{+}$. Suppose that $\int_{D} m_{x} \pi(d x)$ is a $\sigma$-finite, hence excessive, measure and that $\xi \leftarrow \int_{D} m_{x} \pi(d x)$. Then there exists a family $\left(\xi_{x}: x \in D\right)$ of excessive measures such that (i) $\xi_{x} \leftarrow m_{x}, \forall x \in D$, (ii) $x \rightarrow \xi_{x}(f)$ is D-measurable for $f \in \mathcal{E}^{+}$, and (iii) $\xi=\int_{D} \xi_{x} \pi(d x)$.

If $\xi \leq \int m_{x} \pi(d x)$, then $\left(\xi_{x}\right)$ can be chosen such that $\xi_{x} \leq m_{x}$ for each $x \in D$.

PROOF. First suppose that $\xi \leq m \equiv \int_{D} m_{x} \pi(d x) \in$ Exc. By (6.37) and (6.41) there is a family $(T(u): u \geq 0)$ of intrinsic, $\left(\mathcal{G}_{t+}^{*}\right)$-optional times such that

$$
\xi=\int_{0}^{\infty} L_{T(u)} m d u
$$

In fact, since $\xi \leq m$, we have $R(\xi-t \cdot m)=0$ if $t>1$ so that the integral in (6.46) can be truncated at $u=1$. For $x \in D$ define $\xi_{x} \in$ Exc by

$$
\xi_{x}=\int_{0}^{1} L_{T(u)} m_{x} d u .
$$

Clearly $\xi_{x} \leq m_{x}$. It is easy to check that if $F \in\left(\mathcal{G}^{0}\right)^{+}$, then both $x \rightarrow Q_{\xi_{x}}(F)$ and $x \rightarrow Q_{m_{x}}(F)$ are $D$-measurable; this measurability holds as well for $F \in\left(\mathcal{G}^{*}\right)^{+}$ since $D$ is complete. In particular, $(x, u) \rightarrow L_{T(u)} m_{x}(f)$ is product measurable on $D \times[0,1]$ if $f \in \mathcal{E}^{+}$. Using Fubini's theorem to integrate (6.47) we see that

$$
\xi(f)=\int_{D} \xi_{x}(f) \pi(d x), \quad f \in \mathcal{E}^{+} .
$$


Thus, the theorem is proved in case $\xi \leq \int m_{x} \pi(d x)$. In the general case we use (6.41) to decompose $\xi$ as $\xi=\sum_{k=1}^{\infty} \xi^{k}, \xi^{k} \in$ Exc, $\xi^{k} \leq m, \forall k \in \mathbf{N}$, where $m=$ $\int_{D} m_{x} \pi(d x)$ as before. By the first part of the proof each $\xi^{k}$ has a representation

$$
\xi^{k}=\int_{D} \xi_{x}^{k} \pi(d x), \quad \xi_{x}^{k} \in \mathrm{Exc}, \quad \xi_{x}^{k} \leq m_{x}, \forall x \in D .
$$

The desired decompositon for $\xi$ now obtains upon setting $\xi_{x}=\sum_{k=1}^{\infty} \xi_{x}^{k}$, taking note of (6.41).

7. Application to balayage. In this final section we give a small sample of further applications of the weak order on Exc. Our main concern is an analogue of the classical maximum principle for potentials of measures.

Consider briefly the classical case. Suppose that the basic process $X$ is a standard process in classical duality with a second standard process $\hat{X}$ relative to an excessive reference measure $m$. (Only in this paragraph do we deviate from previous terminology, using $\hat{X}$ to denote a strong Markov dual for $X$.) Suppose that the potential kernel $U$ is proper. Then $U(x, d y)=u(x, y) m(d y)$, where $u(\cdot, y)$ is excessive (for $X$ ) and $u(x, \cdot)$ is excessive for $\hat{X}$. If $\mu$ is a measure on $(E, \mathcal{E})$ such that $\mu U$ is $\sigma$-finite, then $\mu U$ has a density relative to $m$ given by

$$
\hat{u}(y)=\int_{E} \mu(d x) u(x, y) .
$$

Clearly $\hat{u}$ is excessive for $\hat{X}$. See [6, VI] for details on these matters. Suppose that $\operatorname{supp}(\mu)$, the support of $\mu$, is compact. The bounded maximum principle is the statement

$$
\text { if } \hat{u} \text { is bounded, then } \sup \{\hat{u}(y): y \in E\}=\sup \{\hat{u}(y): y \in \operatorname{supp}(\mu)\} \text {. }
$$

It is known $[\mathbf{7}, 2.1]$ that $(7.2)$ holds provided $\mu$ charges no semipolar set. Also, if the excessive functions of $\hat{X}$ are lower semicontinuous, then (7.2) holds for all $\mu$ with compact support if and only if $\hat{X}$ satisfies Hunt's hypothesis $\mathrm{H}$ (which is the statement that semipolars for $\hat{X}$ are polar for $\hat{X}$ ). See $[\mathbf{7}, 5.3]$.

In our context, $X$ need not have a strong Markov dual so the representation (7.1) is not valid. We have seen in $\S 6$ that if $\mu U \leftarrow m$, then a version $\hat{\phi}$ of $d(\mu U) / d m$ can be chosen so as to be excessive for the moderate Markov dual $\hat{X}$. However, it is the "fine" version $\bar{\phi}$ of $d(\mu U) / d m$ (i.e., the version provided by (4.15)) which is appropriate for our analogue of (7.2).

The following theorem is the fundamental result of this section; it is an analogue of the classical "second maximum principle". It can be deduced (with some effort) from the work of Azema [3] but we prefer to give a direct proof based on an argument of Meyer [34]. See Doob [13, 2.IV.13] and Meyer [34] for related results.

We fix $m \in$ Exc and follow the notation set in previous sections anent $\left(Y, Q_{m}\right)$. In particular, $\left(\hat{X}_{t}, \hat{P}^{x}\right)$ is the moderate Markov dual, relative to $m$, discussed in $\S 4$.

Let $\xi \in$ Exc with $\xi \leftarrow m$ via $\kappa$ and $J$ as in (6.3). Let the densities $\bar{u}$ and $\hat{u}$ be as in the discussion following (6.8). Also, let $\mu U \in$ Pot with $\mu U \leftarrow m$. Since $\mu U$ has zero invariant part, the invariant function $J$ in the representation (6.4) (for $\mu U$ ) can be taken to vanish. Let $\gamma$ denote the HRM in the representation (6.4) for $\mu U$. The analogous densities for $\mu U$ are denoted $\bar{\phi}$ and $\hat{\phi}$. 
(7.3) THEOREM. Suppose that $\mu$ charges no $m$-polar set and that $\mu$ is carried by $A \in \mathcal{E}$. Suppose that $\{\bar{\phi}>\bar{u}\} \cap A$ is m-polar. Then $\{\bar{\phi}>\bar{u}\}$ is m-polar. In particular, $\mu U \leq \xi$.

PROOF. By (3.2b) it suffices to check that

$$
Q_{m}\left(\bar{\phi}\left(\bar{Y}_{T}\right) ; T \in \Lambda\right) \leq Q_{m}\left(\bar{u}\left(\bar{Y}_{T}\right) ; T \in \Lambda\right)
$$

for each $Q_{m}$-copredictable time $T$. Fix such a $T$ and define

$$
\Gamma=\left\{(t, w) \in \Lambda: t \leq T(w), \bar{Y}_{t}(w) \in A\right\}, \quad S=\sup \Gamma .
$$

Clearly $\Gamma \in \hat{p}^{m}$ and so $S$ is a $Q_{m}$-cooptional time in the sense that $\{S<t\} \in$ $\hat{\mathcal{G}}_{t}^{m}, \forall t \in \mathbf{R}$.

Now define a random interval $L$ by

$$
\begin{array}{rlrl}
L(w) & =]-\infty, S(w)] & & \text { if } S(w) \in \Gamma(w), \\
& =]-\infty, S(w)[ & \text { if } S(w) \notin \Gamma(w) .
\end{array}
$$

Since $\mu$ charges no $m$-polars, the HRM $\gamma$ is carried by $\Lambda$ and $\mu$ is the characteristic

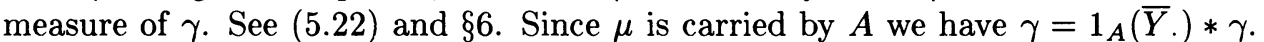
Since $\bar{Y}_{t}(w) \notin A$ if $\left.\left.t \in\right] S(w), T(w)\right]$ and since $\gamma\{S\}=1_{A}\left(\bar{Y}_{S}\right) \gamma\{S\}$ we must have

$$
\gamma(]-\infty, T])=\gamma(L) \text { a.e. } Q_{m} \text {. }
$$

We now approximate $S$ from the left by a sequence $\left(S_{n}\right)$ of $Q_{m}$-copredictable times such that $\bar{Y}_{S_{n}} \in A$ on $\left\{S_{n} \in \Lambda\right\}$ and $\rrbracket-\infty, S_{n} \rrbracket \uparrow L$. First note that $\Gamma \backslash \rrbracket-\infty, S \llbracket(\subset \llbracket S \rrbracket)$ is $Q_{m}$-copredictable with sections of cardinality $\leq 1$. Thus $\Gamma \backslash \rrbracket-\infty, S \llbracket=\llbracket U \rrbracket$ where $U$ is a $Q_{m}$-copredictable time. The set $\llbracket S \rrbracket \backslash \llbracket U \rrbracket$ is thus the graph of a random time $V$ which is $Q_{m}$-cooptional (in the previously noted sense). Moreover if $V(w) \in \mathbf{R}$, then $[V(w)-1 / n, V(w)[\cap \Gamma(w)$ is nonempty for each $n \in \mathbf{N}$. Let $V_{n}$ be a copredictable section of $\llbracket V-1 / n, V \llbracket \cap \Gamma$ so that $V_{n}<V, V_{n} \uparrow V$ as $n \uparrow \infty$. Finally, put $S_{n}=U \vee V_{n}$ so that each $S_{n}$ is $Q_{m}$-copredictable and

(i) $\bar{Y}_{S_{n}} \in A$ on $\left\{S_{n} \in \Lambda\right\}$;

(ii) $S_{n}=S$ if $S \in \Gamma$;

(iii) $S_{n}<S$ if $S \notin \Gamma$;

(iv) $S_{n} \uparrow S$ as $n \uparrow \infty$.

Clearly $\rrbracket-\infty, S_{n} \rrbracket \uparrow L$ as $n \uparrow \infty$. We may now compute

$$
\begin{aligned}
Q_{m}\left(\bar{\phi}\left(\bar{Y}_{T}\right) ; T \in \Lambda\right) & \left.\left.=Q_{m}(\gamma]-\infty, T\right] ; T \in \Lambda\right) \\
& \left.\left.=\uparrow \lim _{n} Q_{m}(\gamma]-\infty, S_{n}\right] ; T \in \Lambda\right) \\
& =\uparrow \lim _{n} Q_{m}\left(\bar{\phi}\left(\bar{Y}_{S_{n}}\right) ; T \in \Lambda\right) \\
& \leq \uparrow \lim _{n} Q_{m}\left(\bar{u}\left(\bar{Y}_{S_{n}}\right) ; T \in \Lambda\right) \leq Q_{m}\left(\bar{u}\left(\bar{Y}_{T}\right) ; T \in \Lambda\right) .
\end{aligned}
$$

For the second equality we have used (7.6) and for the first inequality the fact that $\bar{Y}_{S_{n}} \in A$ on $\left\{S_{n} \in \Lambda\right\}$ and the fact that $\{\bar{\phi}>\bar{u}\} \cap A$ is $m$-polar. Thus (7.4) holds and the theorem is proved.

For $f \in \mathcal{E}^{+}$we define the essential supremum of $f$, relative to the class of $m$-polar sets, as follows:

$$
\|f\|_{m} \equiv \inf \{a>0:\{f>a\} \text { is } m \text {-polar }\} .
$$

Here is our version of the (first) maximum principle, the analogue of (7.2). 
(7.7) COROllary. Let $\mu U \in$ Pot with $\mu U \leftarrow m$. Suppose that $\mu$ charges no $m$-polar set and that $\mu$ is carried by $A \in \mathcal{E}$. Then

$$
\left\|1_{A} \bar{\phi}\right\|_{m}=\|\bar{\phi}\|_{m},
$$

where $\bar{\phi}$ is the "fine" version of $d(\mu U) / d m$ provided by $(4.15)$.

ProOF. Clearly $\left\|1_{A} \bar{\phi}\right\|_{m} \leq\|\bar{\phi}\|_{m}$. If $\left\|1_{A} \bar{\phi}\right\|_{m}=+\infty$ there is nothing more to show. So assume that $a=\left\|1_{A} \bar{\phi}\right\|_{m}<+\infty$. Let $\xi=a \cdot m$ so that a fine version of $d \xi / d m$ is given by $\bar{u}(x) \equiv a$. By hypothesis, $\{\bar{\phi}>a\} \cap A$ is $m$-polar; by (7.3), $\{\bar{\phi}>a\}$ is $m$-polar. That is, $\|\bar{\phi}\|_{m} \leq a$ as required.

We shall use Corollary (7.7) to obtain an analogue of Hunt's balayage theorem. A "crude" result of this type can be found in [18]:

$$
L_{A} m=\inf \{\xi \in \operatorname{Exc}: \xi \geq m \text { on } A\} \quad \text { iff } \quad Q_{m}\left(T_{A} \neq \Pi_{A}\right)=0 .
$$

Here $\Pi_{A}$ is the Lebesgue penetration time of $A$, and inf denotes the greates lower bound in the lattice Exc (with the simple order).

We need one preparatory result. Let $A \in \mathcal{E}$ so that $L_{A} m \leq m$. Let $\bar{\phi}_{A}$ and $\hat{\phi}_{A}$ denote the "fine" and $\left(\hat{P}_{s}\right)$-excessive versions of $d\left(L_{A} m\right) / d m$ respectively. Recall from $\S 6$ that $\bar{\phi}_{A}=\hat{\phi}_{A}+j_{A}$, where $j_{A} \in \mathcal{E}^{+}$and $\left\{j_{A}>0\right\}$ is $m$-semipolar. Also, recall that $A \in \mathcal{E}$ is finely perfect provided $A=\left\{x \in E: P^{x}\left(T_{A}=0\right)=1\right\}$.

(7.10) LemMA. Suppose that $A$ is finely perfect and that $Q_{m}\left(T_{A}=-\infty\right)=0$. Then for all $x$ outside of an m-polar set, $\bar{\phi}_{A}(x)=\hat{\phi}_{A}(x)+1_{A}(x)\left(1-\hat{\phi}_{A}(x)\right)$.

PROOF. Clearly $L_{A} m \leftarrow m$. It is easy to check that in the representation (6.4) we can choose $J \equiv 0$ and

$$
\kappa=\left(1_{\left\{T_{A} \in \mathbf{R}\right\}} \varepsilon_{T_{A}}\right)^{\hat{p}} .
$$

Arguing as in $\S 6$ one sees that if $T$ is a $Q_{m}$-copredictable time, then

$$
\begin{aligned}
& \left.\left.Q_{m}\left(\bar{\phi}_{A}\left(\bar{Y}_{T}\right) ; T \in \Lambda\right)=Q_{m}(\kappa]-\infty, T\right] ; T \in \Lambda\right) ; \\
& Q_{m}\left(\hat{\phi}_{A}\left(\bar{Y}_{T}\right) ; T \in \Lambda\right)=Q_{m}(\kappa]-\infty, T[T \in \Lambda) .
\end{aligned}
$$

Also, by (7.11) and (7.13),

$$
Q_{m}\left(\hat{\phi}_{A}\left(\bar{Y}_{T}\right) ; T \in \Lambda\right)=Q_{m}\left(T_{A}<T ; T \in \Lambda\right) .
$$

As in the proof of (6.16), we can assume that $T=-\infty$ if $T \notin \Lambda$; in this case the condition $T \in \Lambda$ can be dropped in (7.12)-(7.14) if we observe the convention that $\bar{Y}_{-\infty}=\Delta$.

Now fix a $Q_{m}$-copredictable time $T$. Since $Q_{m}$ is $\sigma$-finite on $\hat{\mathcal{G}}_{T+}^{m} \cap\{T \in \Lambda\}$ we may assume that $Q_{m}(T \in \Lambda)<\infty$. We also assume that $T=-\infty$ if $T \notin \Lambda$. Let $\left(S_{n}\right)$ be a decreasing sequence of $Q_{m}$-predictable times with $S_{n}>T$ and $S_{n} \downarrow T$ as $n \uparrow \infty$. Using (7.12)-(7.14) and dominated convergence we compute

$$
\begin{aligned}
Q_{m}\left(\bar{\phi}_{A}\left(\bar{Y}_{T}\right)\right) & \left.\left.=Q_{m}(\kappa]-\infty, T\right]\right)=\lim _{n} Q_{m}(\kappa]-\infty, S_{n}[) \\
& =\lim _{n} Q_{m}\left(\hat{\phi}_{A}\left(\bar{Y}_{S_{n}}\right)\right) \\
& =\lim _{n} Q_{m}\left(Y_{t} \in A \text { for some } t<S_{n}\right) \\
& =Q_{m}\left(Y_{t} \in A \text { for some } t \leq T\right) \\
& =Q_{m}\left(T_{A}<T\right)+Q_{m}\left(\left\{T_{A}<T\right\}^{c} ; \bar{Y}_{T} \in A\right) \\
& =Q_{m}\left(\hat{\phi}_{A}\left(\bar{Y}_{T}\right)\right)+Q_{m}\left(\left(1-\hat{\phi}_{A}\left(\bar{Y}_{T}\right)\right) 1_{A}\left(\bar{Y}_{T}\right)\right) .
\end{aligned}
$$


By $(3.2 \mathrm{~b})$ and $(3.22)$ we have $\bar{\phi}_{A}+\hat{\phi}_{A}=\left(1-\hat{\phi}_{A}\right) 1_{A}$ off an $m$-polar set as required.

Let $A \in \mathcal{E}$ be finely perfect and suppose that $Q_{m}\left(T_{A}=\alpha\right)=0$. Then $L_{A} m$ is a potential, say $L_{A} m=\mu_{A} U$. Moreover $\mu_{A}$ charges no $m$-polar set, and $\mu_{A}$ is carried by $A$ (since $A$ is finely closed). See [19 or $\mathbf{3 0}]$. In the following result $d \xi / d m$ is taken to be the fine version provided by (4.15).

(7.15) COROllary. With $A$ as above,

$$
L_{A} m=\inf \left\{\xi \in \operatorname{Exc}: \xi \leftarrow m,\left\|1_{A}[d \xi / d m]^{-1}\right\|_{m} \leq 1\right\} .
$$

PROOF. Since $L_{A} m \leq m$ we have $L_{A} m \leftarrow \cdot m$, and we may assume that $\bar{\phi}_{A} \leq 1$ everywhere. By (7.10), $\left\{\bar{\phi}_{A}<1\right\} \cap A$ is $m$-polar. Thus $\left\|1_{A}\left[\bar{\phi}_{A}\right]^{-1}\right\|_{m}=1$, and so $L_{A} m$ is an element of the set on the right side of (7.16). Let $\xi \in$ Exc be any element of this set. Write $\bar{u}=d \xi / d m$. Then there is a Borel $m$-polar set $N \subset A$ such that $x \in A \backslash N \Rightarrow \bar{u}(x) \geq 1=\bar{\phi}_{A}(x)$. By (7.3), $\left\{\bar{u}<\bar{\phi}_{A}\right\}$ is $m$-polar; in particular $\xi \geq L_{A} m$.

Let $A$ be as for Corollary (7.15). Then $Q_{m}\left(T_{A}=-\infty\right) \leq Q_{m}\left(T_{A}=\alpha\right)=0$ so that $A$ is $m$-cotransient in the terminology of [30]. The measure $\mu_{A}$ is the cocapacitary measure of $A$, and its mass $\mu_{A}(1)$ is the cocapacity of $A$, denoted $\hat{C}(A)$. See [30]. Let $D$ denote the class of measures $\mu$ on $(E, \mathcal{E})$ such that $\mu$ charges no $m$-polar set, $\mu$ is carried by $A$, and $\mu U \leq m$. Clearly $\mu_{A} \in D$. If $\mu \in D$ is arbitrary let $\bar{u}$ denote the fine version of $d(\mu U) / d m$ so that $\bar{u} \leq 1$. Arguing as in the proof of (7.15) one sees that $\left\{\bar{u}>\bar{\phi}_{A}\right\}$ is $m$-polar. Thus $\mu U \leq \mu_{A} U=L_{A} m$. It follows that $\mu(1) \leq \mu_{A}(1)=\hat{C}(A)$. We have proved the following analogue of a classical result

$$
\hat{C}(A)=\sup \{\mu(1): \mu \in D\} .
$$

Without doubt the hypotheses we have imposed in this section can be weakened. We hope to return to these matters, and to consider connections with energy, in a future publication.

Appendix. In this section we prove Theorem 4.6. We shall use the notation of the body of the paper without special mention. As noted in $\S 4$, the argument used is adapted from Azema [2] and Jeulin [32].

Firstly, let us extend the notion of $m$-polar set, defined prior to (3.22) only for Borel sets. For a general set $A \subset E$ we say that $A$ is $m$-polar provided there is a Borel $m$-polar set $A^{\prime} \supset A$. Clearly $A \in \mathcal{E}$ is $m$-polar if and only if $P^{m}\left(T_{A}<\infty\right)=0$, where $T_{A}=\inf \left\{t>0: X_{t} \in A\right\}$. Define

$$
J(A)=P^{\mu}\left(T_{A}<\infty\right), \quad A \in \mathcal{E},
$$

where $\mu$ is a probability on $\mathcal{E}$ equivalent to $m$. Thus $B \in \mathcal{E}$ is $m$-polar if and only if $J(B)=0$. Let $R$ denote the class of open sets in a Ray-Knight compactification $\bar{E}$ of $E_{\Delta}$, relative to the usual extension of $\left(P_{s}\right)$ to a Markov semigroup on $\left(E_{\Delta}, \mathcal{E}_{\Delta}\right)$. Denote $J^{*}$ by

$$
J^{*}(A)=\inf \{J(G \cap E): G \in R, G \supset A\}, \quad A \subset E .
$$

Shih's Theorem (see [24]) implies that $J^{*}$ is a strongly subadditive Choquet capacity. In particular, if $A \subset E$ is a Souslin subset of $E$ (hence capacitable), then $A$ is 
$m$-polar if and only if $J(B)=0$ for all Borel sets $B \subset A$. See [12] for details on such matters.

Next, let $d$ be a bounded (by 1) metric on $E$ compatible with its topology; set $d(x, \Delta)=2$ if $x \in E$, and $d(\Delta, \Delta)=0$. Define a metric $\rho$ on $\hat{\Omega}$ by

$$
\rho\left(w, w^{\prime}\right)=\int_{-\infty}^{0} e^{t} d\left(w_{t}, w_{t}^{\prime}\right) d t .
$$

The metric topology induced on $\hat{\Omega}$ by $\rho$ has $\hat{\mathcal{f}}^{0}$ for its Borel $\sigma$-field. The completion $\bar{\Omega}$ of $\hat{\Omega}$ relative to $\rho$ is a compact metric space and $\hat{\Omega}$ is a coanalytic subset of $\bar{\Omega}$ (see [12, IV (19)]). If $\overline{\mathcal{F}}$ denotes the Borel subsets of $\bar{\Omega}$, then $\hat{\mathcal{F}}^{0}=\overline{\mathcal{F}} \cap \hat{\Omega}$. The dominated convergence theorem shows that if $w \in W$ (resp. $w \in \hat{\Omega}$ ), then $t \rightarrow \hat{\tau}_{t} w$ (resp. $\left.s \rightarrow \hat{\theta}_{s} w\right)$ is $\rho$-continuous as a map from $]-\infty, \beta(w)[$ (resp. $] 0,+\infty[$ ) into $\hat{\Omega}$.

We now produce a kernel $L=L(x, d w)$ from $\left(E_{\Delta}, \mathcal{E}_{\Delta}\right)$ to $\left(\hat{\Omega}, \hat{\mathcal{F}}^{0}\right)$ such that $(4.2)$ holds with $\hat{P}^{x}$ replaced by $L(x, \cdot)$. The desired kernel $\hat{P}^{x}$ will then be obtained by regularizing $L$ so that (4.4) holds as well. See (A.3). Let $C(\bar{\Omega})$ denote the class of real-valued continuous functions on $\bar{\Omega}$. Given $F \in C(\bar{\Omega})$ note that $t \rightarrow$ $F\left(\hat{\tau}_{t} w\right)$ is continuous on $]-\infty, \beta(w)$ [ and is there identical with $\left(F 1_{\hat{\Omega}}\right) \circ \hat{\tau}_{t}$. Thus $t \rightarrow 1_{\rrbracket-\infty, \beta \llbracket}(t)\left(F 1_{\hat{\Omega}}\right) \circ \hat{\tau}_{t}$ is rc and evidently adapted to $\left(\mathcal{G}_{t+}^{0}\right)$. Consider now the process

$$
Z_{t}^{F}={ }^{\hat{p}}\left(1_{\rrbracket-\infty, \beta \llbracket}\left(F 1_{\hat{\Omega}}\right) \circ \hat{\tau}_{.}\right)_{t} 1_{\Lambda}(t) .
$$

By the right continuity of $t \rightarrow 1_{\rrbracket-\infty, \beta \llbracket}(t)\left(F 1_{\hat{\Omega}}\right) \circ \hat{\tau}_{t}$ (and the fact that this process is constant on ] $-\infty, \alpha(w)]$ ), it follows from (3.4) that $Z^{F} \in \mathcal{O}^{m}$. Thus $Z^{F} \in \mathcal{O}^{m} \cap \hat{P}^{m}$ and clearly $Z^{F}$ is homogeneous. By $(3.21), Z^{F}$ has the form $f^{F}\left(\bar{Y}_{t}\right)$ for some $f^{F} \in b \mathcal{E}$; moreover, $f^{F}$ is uniquely determined up to an $m$-polar set. The existence of $f^{F}$ for arbitrary $F \in b \overline{\bar{F}}\left(Z^{F}\right.$ being defined by (A.1)) now follows by a monotone class argument. It follows that the mapping $F \rightarrow f^{F}$, from $b \overline{\mathcal{F}}$ to $b \mathcal{E}$ is a "pseudokernel" from $(E, \mathcal{E})$ to $(\bar{\Omega}, \overline{\mathcal{F}})$, relative to the class of $m$-polar sets. By Corollary (3.2) of [23] there is a sub-Markov kernel $L_{0}=L_{0}(x, d w)$ from $(E, \mathcal{E})$ to $(\bar{\Omega}, \overline{\mathcal{F}})$ such that for each $F \in b \overline{\bar{F}}$, the set $\left\{x \in E: L_{0}(x, F) \neq f^{F}(x)\right\}$ is a Borel $m$-polar set. It follows from (A.1) that for any $F \in b \overline{\mathcal{F}}^{+}$and any $Q_{m}$-copredictable time $T$,

$$
Q_{m}\left(F \circ \hat{\tau}_{T} ; T \in \Lambda\right)=Q_{m}\left(L_{0}\left(\bar{Y}_{T}, F\right) ; T \in \Lambda\right) .
$$

Take $F=1_{\bar{\Omega}}$ in (A.2) to see that the Borel set $\left\{x \in E: L_{0}(x, \bar{\Omega})<1\right\}$ is $m$-polar. Note also that if $C=\left\{x \in E: L_{0}(x, \bar{\Omega} \backslash \hat{\Omega})>0\right\}$, then $C$ is Souslin in $E$ [33, p. 236]. Now let $B \subset C$ with $B \in \mathcal{E}$, and take $F=1_{\bar{\Omega} \backslash \hat{\Omega}}$ in (A.2):

$$
\begin{aligned}
0 & =Q_{m}\left(1_{\bar{\Omega} \backslash \hat{\Omega}} \circ \hat{\tau}_{T} ; \bar{Y}_{T} \in B, T \in \Lambda\right) \\
& =Q_{m}\left(L_{0}\left(\bar{Y}_{T}, 1_{\bar{\Omega} \backslash \hat{\Omega}}\right) ; \bar{Y}_{T} \in B, T \in \Lambda\right) .
\end{aligned}
$$

That is, $B$ is $m$-polar. By the discussion in the second paragraph of this appendix, $C$ is $m$-polar. Finally, enclose $C \cup\left\{x: L_{0}(x, \bar{\Omega})<1\right\}$ in a Borel $m$-polar set $A$ and define

$$
\begin{aligned}
L(x, F) & =L_{0}(x, F), \quad x \in E \backslash A, \\
& =\varepsilon_{[\Delta]}(F), \quad x \in A \cup\{\Delta\} .
\end{aligned}
$$


Clearly $L$ is a Markov kernel from $\left(E_{\Delta}, \mathcal{E}_{\Delta}\right)$ to $(\bar{\Omega}, \overline{\mathcal{F}})$ such that $L(x, \cdot)$ is carried by $\hat{\Omega}$ for each $x \in E_{\Delta}$. Since $\hat{\mathcal{F}}^{0}=\overline{\mathcal{F}} \cap \hat{\Omega}$, we may regard $L$ as a kernel from $\left(E_{\Delta}, \mathcal{E}_{\Delta}\right)$ to $\left(\hat{\Omega}, \hat{\mathcal{F}}^{0}\right)$. Finally, let us check that $(4.2)$ holds: using the fact that $\hat{\tau}_{t} w=[\Delta]$ if $t \leq \alpha(w)$, if $T$ is $Q_{m}$-copredictable then by (A.2),

$$
\begin{aligned}
Q_{m}( & \left.F \circ \hat{\tau}_{T} ;-\infty<T<\beta\right) \\
& =Q_{m}\left(F \circ \hat{\tau}_{T} ; T \in \Lambda\right)+Q_{m}(F([\Delta]) ;-\infty<T \leq \alpha, T \notin \Lambda) \\
& =Q_{m}\left(L_{0}\left(\bar{Y}_{T}, F\right) ; T \in \Lambda\right)+Q_{m}\left(\varepsilon_{[\Delta]}(F) ;-\infty<T \leq \alpha, T \notin \Lambda\right) \\
& =Q_{m}\left(L\left(\bar{Y}_{T}, F\right) ;-\infty<T<\beta\right) .
\end{aligned}
$$

Theorem (4.6) is an immediate corollary of the following result.

(A.3) PROPOSITION. (a) Let $L^{\prime}$ be a second Markov kernel from $\left(E_{\Delta}, \mathcal{E}_{\Delta}\right)$ to $\left(\hat{\Omega}, \hat{\mathcal{F}}^{0}\right)$ such that $(4.2)$ holds with $L^{\prime}(x, \cdot)$ in place of $\hat{P}^{x}$. Then $\{x: L(x, \cdot) \neq$ $\left.L^{\prime}(x, \cdot)\right\}$ is a Borel m-polar set.

(b) There exists a Borel m-polar set $N$ such that if $\hat{P}^{x}$ is defined by

$$
\begin{aligned}
\hat{P}^{x} & =L(x, \cdot), \quad x \in E \backslash N, \\
& =\varepsilon_{[\Delta]}, \quad x \in N \cup\{\Delta\},
\end{aligned}
$$

then $\left(\hat{P}^{x}\right)$ satisfies Definition (4.1).

Proof. (a) Extend $L^{\prime}$ to a kernel from $\left(E_{\Delta}, \mathcal{E}_{\Delta}\right)$ to $(\bar{\Omega}, \overline{\mathcal{F}})$ by setting $L^{\prime \prime}(x, F)=$ $L^{\prime}\left(x, F 1_{\hat{\Omega}}\right)$ for $F \in \overline{\mathcal{F}}$ (recall that $\left.\hat{\mathcal{F}}^{0}=\overline{\bar{F}} \cap \hat{\Omega}\right)$. If $D$ is a total subset of $C(\bar{\Omega})$ (with the uniform norm), then clearly

$$
\left\{x: L(x, \cdot) \neq L^{\prime}(x, \cdot)\right\}=\bigcup_{F \in D}\left\{x: L(x, F) \neq L^{\prime \prime}(x, F)\right\}
$$

which is a Borel $m$-polar set.

(b) Let $b P$ denote the class of bounded predictable processes relative to $\left(\hat{\mathcal{F}}_{t}^{0}\right)$. Given $A \in b P$ and $B \in b \hat{f}^{0}$ we claim that for each $t>0$ the Borel set

$$
\left\{x: L\left(x, A_{t} B \circ \hat{\theta}_{t}\right) \neq L\left(x, A_{t} L\left(\hat{X}_{t}, B\right)\right)\right\}
$$

is $m$-polar. It suffices to prove (A.4) when $A$ and $B$ are positive; if $T$ is $Q_{m^{-}}$ copredictable then $T-t$ is $Q_{m}$-copredictable for $t>0$ and $A_{t} \circ \hat{\tau}_{T} 1_{\{T<\beta\}}$ is $\hat{\mathcal{G}}_{(T-t)+^{-}}^{m}$ measurable. Thus

$$
\begin{aligned}
Q_{m}( & \left.L\left(\bar{Y}_{T}, A_{t} B \circ \hat{\theta}_{t}\right) ; T<\beta\right) \\
& =Q_{m}\left(A_{t} \circ \hat{\tau}_{T} B \circ \hat{\theta}_{t} \circ \hat{\tau}_{T} ; T<\beta\right) \\
& =Q_{m}\left(A_{t} \circ \hat{\tau}_{T} B \circ \hat{\tau}_{T-t} ; T<\beta\right)=Q_{m}\left(A_{t} \circ \hat{\tau}_{T} L\left(\bar{Y}_{T-t}, B\right), T<\beta\right) \\
& =Q_{m}\left(\left\{A_{t} L\left(\hat{X}_{t}, B\right)\right\} \circ \hat{\tau}_{T} ; T<\beta\right)=Q_{m}\left(L\left(\bar{Y}_{T}, A_{t} L\left(\hat{X}_{t}, B\right)\right) ; T<\beta\right) .
\end{aligned}
$$

The claim that (A.4) is $m$-polar now follows from (3.2b) and (3.22). Now fix $B \in C(\bar{\Omega})$ and consider the set

$$
\Gamma=\left\{w \in W: t \rightarrow L\left(\bar{Y}_{t}, B\right) \text { is rc on }\right]-\infty, \beta[\} .
$$

The set $\Gamma$ is co-Souslin in $W$ (see [12]) and by a result of Meyer cited earlier, $\left\{x \in E: L\left(x, \Gamma^{c} \cap \hat{\Omega}\right)>0\right\}$ is Souslin in $E$. We claim that

$$
\left\{x \in E: L\left(x, \Gamma^{c} \cap \hat{\Omega}\right)>0\right\} \text { is } m \text {-polar. }
$$


By the preceding remarks, to show this, it suffices to show that for any $Q_{m^{-}}$ copredictable time $T$,

$$
Q_{m}\left(L\left(\bar{Y}_{T}, \Gamma^{c} \cap \hat{\Omega}\right),-\infty<T<\beta\right)=0 .
$$

Now $t \rightarrow L\left(\bar{Y}_{t}, B\right)$ is a version of the $Q_{m}$-copredictable projection of the rc process $t \rightarrow 1_{\rrbracket-\infty, \beta \llbracket}(t) B \circ \hat{\tau}_{t}$. Thus $t \rightarrow L\left(\bar{Y}_{t}, B\right)$ is rc a.e., $Q_{m}$. Thus, the left side of (A.7) equals

$$
\begin{aligned}
Q_{m}( & \left.1_{\Gamma^{c} \cap \hat{\Omega}^{\circ}} \circ \hat{\tau}_{T} ;-\infty<T<\beta\right) \\
& =Q_{m}\left(1_{\Gamma^{c}} \circ \hat{\tau}_{T} ;-\infty<T<\beta\right) \\
& =Q_{m}\left(t \rightarrow L\left(\bar{Y}_{t}, B\right) \text { is not } \mathrm{rc} \text { on }\right]-\infty, T[;-\infty<T<B) \\
& =0,
\end{aligned}
$$

and (A.6) holds as claimed.

Since $P$ is separable we may choose a sequence of bounded, positive, left continuous processes $\left(A^{k}: k \geq 1\right)$ which generate $P$. Let $\left(B^{n}: n \geq 1\right)$ be a sequence from $C(\bar{\Omega})^{+}$whose linear span is dense in $C(\bar{\Omega})$. Then

(i) For each $n \geq 1$ we may choose a Borel $m$-polar set $N_{n}$ such that for $x \notin$ $N_{n}, t \rightarrow L\left(\hat{X}_{t}, B^{n}\right)$ is lc on $] 0,+\infty[$ a.s. $L(x, \cdot)$. See (A.6).

(ii) By the discussion concerning (A.4), for each $n \geq 1, k \geq 1$, and rational $t>0$, the set

$$
N_{n, k, t}=\left\{x: L\left(x, A_{t}^{k} B^{n} \circ \hat{\theta}_{t}\right) \neq L\left(x, L\left(\hat{X}_{t}, B^{n}\right) A_{t}^{k}\right)\right\}
$$

is a Borel $m$-polar set. Put

$$
N=\left(\bigcup_{n \geq 1} N_{n}\right) \cup\left(\bigcup_{n, k \geq 1, t>0, \text { rational }} N_{n, k, t}\right)
$$

so that $N$ is a Borel $m$-polar set. If $x \in E \backslash N$, then

$$
L\left(x, A_{t}^{k} B^{n} \circ \hat{\theta}_{t}\right)=L\left(x, A_{t}^{k} L\left(\hat{X}_{t}, B^{n}\right)\right)
$$

for all $k \geq 1, n \geq 1$ and all $t>0$ (since both sides of (A.9) are left continuous by (i) above). Since $\left(A^{k}: k \geq 1\right)$ generates $b P$, it follows that if $x \in E \backslash N$, then $t \rightarrow L\left(\hat{X}_{t}, B^{n}\right)$ is a version of the $L(x, \cdot)$-predictable projection of $t \rightarrow B^{n} \circ \hat{\theta}_{t}$. Since $\left(B^{n}: n \geq 1\right)$ is total in $C(\bar{\Omega})$, the previous sentence remains true if $B^{n}$ is replaced by an arbitrary $B \in C(\bar{\Omega})$. By a monotone class argument, since $\hat{\mathcal{F}}^{0}=\overline{\mathcal{F}} \cap \hat{\Omega}$,

$$
L\left(x, B \circ \hat{\theta}_{T} ; 0<T<\infty\right)=L\left(x, L\left(\hat{X}_{T}, B\right) ; 0<T<\infty\right)
$$

whenever $x \in E \backslash N, B \in b \hat{\mathcal{F}}^{0}$, and $T$ is a $\left(\hat{\mathcal{F}}_{t}^{0}\right)$-predictable time. The Borel $m$-polar set $N$ has the properties claimed in the statement of Proposition (A.3b). Indeed, (A.10) implies (4.4) while (4.2) holds for $\left(\hat{P}^{\cdot}\right)$ since it holds for $L$ by construction.

\section{REFERENCES}

1. B. W. Atkinson and J. B. Mitro, Applications of Revuz and Palm type measures for additive functionals in weak duality, Seminar on Stochastic Processes, 1982, Birkhäuser, Boston, Mass., 1983, pp. 23-49.

2. J. Azema, Theorie generale des processus et retournement du temps, Ann. Sci. École Norm. Sup. 6 (1973), 459-519. 
3. _ Quelques applications de la theorie generale des processus. I, Invent. Math. 18 (1972), 293-336.

4. J. Azema and Th. Jeulin, Precisions sue la mesure de Follmer, Ann. Inst. H. Poincaré 12 (1976), 257-283.

5. A. Benveniste, Processus stationaires et mesures de Palm du flot special sous une fonction, Lecture Notes in Math., vol. 465, Springer-Verlag, Berlin and New York, 1975, pp. 97-153.

6. R. M. Blumenthal and R. K. Getoor, Markov processes and potential theory, Academic Press, New York, 1968.

7. _ Dual processes and potential theory, Proc. 12th Biennial Sem. Canad. Math. Soc., Canad. Math. Soc., Montreal, 1970, pp. 137-156.

8. N. Boboc, G. Bucur and A. Cornea, Order and convexity in potential theory: H-cones, Lecture Notes in Math., vol. 853, Springer-Verlag, Berlin and New York, 1981.

9. K. L. Chung and J. Glover, Left continuous moderate Markov processes, Z. Wahrsch. Verw. Gebiete 49 (1979), 237-248.

10. K. L. Chung and J. B. Walsh, To reverse a Markov process, Acta Math. 123 (1969), 225-251.

11. E. Cinlar, J. Jacod, P. Protter and M. Sharpe, Semimartingales and Markov processes, Z. Wahrsch. Verw. Gebiete 54 (1980), 161-219.

12. C. Dellacherie and P.-A. Meyer, Probabilites et potentiel, vols. I, II, III, Hermann, Paris, 1975, 1980, 1983.

13. J. L. Doob, Classical potential theory and its probabilistic counterpart, Springer-Verlag, Berlin and New York, 1984.

14. E. B. Dynkin, Markov systems and their additive functionals, Ann. Probab. 5 (1977), 653-677.

15. __ An application of flows to time shift and time reversal in stochastic processes, Trans. Amer. Math. Soc. 287 (1985), 613-620.

16. __ Minimal excessive measures and functions, Trans. Amer. Math. Soc. 258 (1980), 217244.

17. E. B. Dynkin and R. K. Getoor, Additive functionals and entrance laws, J. Funct. Anal. 62 (1985), 221-265.

18. P. J. Fitzsimmons, Penetration times and Skorohod stopping, Manuscript, 1986.

19. P. J. Fitzsimmons and B. Maisonneuve, Excessive measures and Markov processes with random birth and death, Prob. Th. Rel. Fields 72 (1986), 319-336.

20. M. A. Garcia Alvarez and P.-A. Meyer, Une theorie de la dualite a ensemble polaire pres, Ann. Probab. 1 (1973), 207-222.

21. D. Geman and J. Horowitz, Remarks on Palm measures, Ann. Inst. H. Poincaré 9 (1973), 215-232.

22. __ Polar sets and Palm measures in the theory of flows, Trans. Amer. Math. Soc. 208 (1975), 141-159.

23. R. K. Getoor, On the construction of kernels, Lecture Notes in Math., vol. 465, SpringerVerlag, Berlin and New York, 1975, pp. 441-463.

24. __ Markov processes: Ray processes and right processes, Lecture Notes in Math., vol. 440, Springer-Verlag, Berlin and New York, 1975.

25. - Measures that are translation ivariant in one coordinate, Seminar on Stochastic Processes, 1986, Birkhäuser, Boston, Mass., 1987, pp. 31-34.

26. R. K. Getoor and J. Glover, Riesz decompositions in Markov process theory, Trans. Amer. Math. Soc. 285 (1984), 107-132.

27. __ Markov processes with identical excessive measures, Math. Z. 184 (183), 287-300.

28. __ Constructing Markov processes with random times of birth and death, Seminar on Stochastic Processes, 1986, Birkhäuser, Boston, Mass., 1987, pp. 35-69.

29. R. K. Getoor and M. J. Sharpe, Naturality, standardness, and weak duality for Markov processes, Z. Wahrsch. Verw. Gebiete 67 (1984), 1-62.

30. R. K. Getoor and J. Steffens, Capacity theory without duality, Prob. Th. Rel. Fields 73 (1986), 415-445.

31. S. K. Kuznetsov, Construction of Markov processes with random times of birth and death, Theory Probab. Appl. 18 (1974), 571-574.

32. Th. Jeulin, Compactification de Martin d'un processus droit, Z. Wahrsch. Verw. Gebiete 42 (1978), 229-260. 
33. P.-A. Meyer, Le retournement du temps, d'apres Chung et Walsh, Lecture Notes in Math., vol. 191, Springer-Verlag, Berlin and New York, 1971, pp. 211-236.

34. __ Resultats d'Azema en "theorie generale des processus, Lecture Notes in Math., vol. 258, Springer-Verlag. Berlin and New York, 1972, pp. 180-197.

35. P.-A. Meyer and J. de Sam Lazaro, Questions de theorie des flots, Lecture Notes in Math., vol. 465, Springer-Verlag, Berlin and New York, 1975, pp. 2-96.

36. J. B. Mitro, Dual Markov processes: Construction of a useful auxiliary process, Z. Wahrsch. Verw. Gebiete 47 (1979), 139-156.

37. __ Dual Markov functionals: Application of a useful auxiliary process, Z. Wahrsch. Verw. Gebiete 48 (1979), 97-114.

38. __ Balayage and exit systems for dual Markov processes, 1982 (Preprint).

39. D. Revuz, Measures associees aux fonctionelles de Markov. I, Trans. Amer. Math. Soc. 148 (1970), 501-531.

40. R. T. Smythe and J. B. Walsh, The existence of dual processes, Invent. Math. 19 (1973), $113-148$.

41. V. Strassen, The existence of probability measures with given marginals, Ann. Math. Statist. 36 (1965), 423-439.

42. D. Heath, Skorohod stopping via potential theory, Lecture Notes in Math., vol. 381, SpringerVerlag, Berlin and New York, 1974, pp. 150-154.

43. H. Rost, The stopping distributions of a Markov process, Invent. Math. 14 (1971), 1-16.

Department of Mathematics, University of California, San Diego, La Jolla, CALIFORNIA 92093 